\title{
1 COVID-19 Prognostic Modeling Using CT \\ 2 \\ Radiomic Features and Machine Learning Algorithms: Analysis of a Multi-Institutional Dataset of 14,339 Patients
}

Isaac Shiri , Yazdan Salimi ${ }^{1}$, Masoumeh Pakbin ${ }^{2}$, Ghasem Hajianfar ${ }^{3}$, Atlas Haddadi Avval ${ }^{4}$, Amirhossein Sanaat , Shayan Mostafaei ${ }^{5,6}$, Azadeh Akhavanallaf ${ }^{1}$, Abdollah Saberi ${ }^{1}$, Zahra Mansouri ${ }^{1,7}$, Dariush Askari, Mohammadreza Ghasemian ${ }^{9}$, Ehsan Sharifipour ${ }^{10}$, Saleh Sandoughdaran ${ }^{11}$, Ahmad Sohrabi ${ }^{12}$, Elham Sadati ${ }^{13}$, Somayeh Livani $^{14}$, Pooya Iranpour ${ }^{15}$, Shahriar Kolahi ${ }^{16}$, Maziar Khateri ${ }^{17}$, Salar Bijari ${ }^{13}$, Mohammad Reza Atashzar $^{18}$, Sajad P. Shayesteh ${ }^{19}$, Bardia Khosravi ${ }^{35}$, Mohammad Reza Babaei ${ }^{20}$, Elnaz Jenabi ${ }^{21}$, Mohammad Hasanian $^{22}$, Alireza Shahhamzeh ${ }^{23}$, Seyed Yaser Foroghi Gholami ${ }^{23}$, Abolfazl Mozafari ${ }^{24}$, Arash Teimouri ${ }^{15}$, Fatemeh Movaseghi $^{24}$, Azin Ahmari ${ }^{25}$, Neda Goharpey ${ }^{26}$, Rama Bozorgmehr ${ }^{27}$, Hesamaddin Shirzad-Aski ${ }^{28}$, Rozbeh Mortazavi $^{29}$, Jalal Karimi ${ }^{30}$, Nazanin Mortazavi ${ }^{31}$, Sima Besharat ${ }^{32}$, Mandana Afsharpad ${ }^{12}$, Hamid Abdollahi $^{33}$, Parham Geramifar ${ }^{34}$, Amir Reza Radmard ${ }^{35}$, Hossein Arabi ${ }^{1}$, Kiara Rezaei-Kalantari ${ }^{3}$, Mehrdad Oveisi $^{36}$, Arman Rahmim ${ }^{37,38} \&$ Habib Zaidi ${ }^{1,39,40,41}$

1. Division of Nuclear Medicine and Molecular Imaging, Geneva University Hospital, Geneva, 1211, Switzerland

2. Imaging Department, Qom University Of Medical Sciences, Qum, Iran

3. Rajaie Cardiovascular, Medical \& Research Center, Iran University of Medical Science, Tehran, Iran

4. School of Medicine, Mashhad University of Medical Sciences, Mashhad, Iran

5. Division of Clinical Geriatrics, Department of Neurobiology, Care Sciences and Society, Karolinska Institute, Stockholm, Sweden

6. Department of Biostatistics, Kermanshah University of Medical Sciences, Kermanshah, Iran

7. Department of Biomedical engineering and medical physics, Shahid Beheshti University of Medical Sciences, Tehran, Iran

8. Department of Radiology Technology, Shahid Beheshti University of Medical Sciences, Tehran, Iran

9. Department of Radiology, Shahid Beheshti Hospital, Qom University of Medical Sciences, Qum, Iran

10. Neuroscience Research Center, Qom University of Medical Sciences, Qum, Iran

11. Men's Health and Reproductive Health Research Center, Shahid Beheshti University of Medical Sciences, Tehran, Iran

12. Cancer control research center, cancer control foundation, Iran University of medical sciences, Tehran, Iran

13. Department of Medical Physics, Faculty of Medical Sciences, Tarbiat Modares University, Tehran, Iran

14. Clinical Research Development Unit (CRDU), Sayad Shirazi Hospital, Golestan University of Medical Sciences, Gorgan, Iran

15. Medical Imaging Research Center, Department of Radiology, Shiraz University of Medical Sciences, Shiraz, Iran

16. Department of Radiology, School of Medicine, Advanced Diagnostic and Interventional Radiology Research Center (ADIR), Imam Khomeini Hospital, Tehran University of Medical Sciences, Tehran, Iran

NOTE: This preprint reports new research that has not been certified by peer review and should not be used to guide clinical practice. 
medRxiv preprint doi: https://doi.org/10.1101/2021.12.07.21267364; this version posted December 7, 2021. The copyright holder for this preprint (which was not certified by peer review) is the author/funder, who has granted medRxiv a license to display the preprint in

It is made available under a CC-BY-NC-ND 4.0 International license.

17. Department of Medical Radiation Engineering, Science and Research Branch, Islamic Azad University, Tehran, Tehran, Iran

18. Department of Immunology, School of Medicine, Fasa University of Medical Sciences, Fasa, Iran

19. Department of Physiology, Pharmacology and medical physics, Alborz University of Medical Sciences, Karaj, Iran

20. Department of interventional radiology, Firouzgar hospital, Iran university of medical sciences, Tehran, Iran

21. Research Centre for Nuclear Medicine, Shariati Hospital, Tehran University of Medical Sciences, Tehran, Iran

22. Department of Radiology, Arak University of Medical Sciences, Arak, Iran

23. Clinical research development center, Qom University of Medical Sciences, Qum, Iran

24. Department of Medical Sciences, Qom Branch, Islamic Azad University, Qum, Iran

25. Ayatolah Khansary hospital, Arak University of Medical Sciences, Arak, Iran

26. Department of radiation oncology, Shohadaye Tajrish Hospital, Shahid Beheshti university of Medical Sciences, Tehran, Iran

27. Clinical Research Development Unit, Shohadaye Tajrish Hospital, shahid Beheshti university of medical sciences, Tehran, Iran

28. Infectious Diseases Research Center, Golestan University of Medical Sciences, Gorgan, Iran

29. Department of Internal Medicine, Shiraz University of Medical Sciences, Shiraz, Iran

30. Department of Infectious Disease, School of Medicine, Fasa University of Medical Sciences, Fasa, Iran

31. Dental Research Center, Golestan University of Medical Sciences, Gorgan, Iran

32. Golestan Research Center of Gastroenterology and Hepatology, Golestan University of Medical Sciences, Gorgan, Iran

33. Department of Radiologic Technology, Faculty of Allied Medical Sciences, Kerman University of Medical Sciences, Kerman, Iran

34. Research Center for Nuclear Medicine, Shariati Hospital, Tehran University of Medical Sciences Tehran, Iran

35. Department of Radiology, Shariati Hospital, Tehran University of Medical Sciences, Tehran, Iran

36. Comprehensive Cancer Centre, School of Cancer \& Pharmaceutical Sciences, Faculty of Life Sciences \& Medicine, King's College London, London, United Kingdom

37. Departments of Radiology and Physics, University of British Columbia, Vancouver BC, Canada

38. Department of Integrative Oncology, BC Cancer Research Centre, Vancouver BC, Canada

39. Geneva University Neurocenter, Geneva University, Geneva, Switzerland

40. Department of Nuclear Medicine and Molecular Imaging, University of Groningen, University Medical Center Groningen, Groningen, Netherlands

41. Department of Nuclear Medicine, University of Southern Denmark, Odense, Denmark

\section{First Author:}

Isaac Shiri, MSc

Geneva University Hospital, Division of Nuclear Medicine and Molecular Imaging,

CH-1211 Geneva, Switzerland

Email: Isaac.shirilord@unige.ch

\section{Corresponding author:}

Habib Zaidi, Ph.D

Geneva University Hospital, Division of Nuclear Medicine and Molecular Imaging

CH-1211 Geneva, Switzerland

Tel: +41223727258

Fax: +41 223727169

email: habib.zaidi@hcuge.ch 


\section{Abstract}

Objective: In this large multi-institutional study, we aimed to analyze the prognostic power of computed tomography (CT)-based radiomics models in COVID-19 patients.

Methods: CT images of 14,339 COVID-19 patients with overall survival outcome were collected from 19 medical centers. Whole lung segmentations were performed automatically using a previously validated deep learning-based model, and regions of interest were further evaluated and modified by a human observer. All images were resampled to an isotropic voxel size, intensities were discretized into 64-binning size, and 105 radiomics features, including shape, intensity, and texture features were extracted from the lung mask. Radiomics features were normalized using Z-score normalization. High-correlated features using Pearson $\left(\mathrm{R}^{2}>0.99\right)$ were eliminated. We applied the Synthetic Minority Oversampling Technique (SMOT) algorithm in only the training set for different models to overcome unbalance classes. We used 4 feature selection algorithms, namely Analysis of Variance (ANOVA), KruskalWallis (KW), Recursive Feature Elimination (RFE), and Relief. For the classification task, we used seven classifiers, including Logistic Regression (LR), Least Absolute Shrinkage and Selection Operator (LASSO), Linear Discriminant Analysis (LDA), Random Forest (RF), AdaBoost (AB), Naïve Bayes (NB), and Multilayer Perceptron (MLP). The models were built and evaluated using training and testing sets, respectively. Specifically, we evaluated the models using 10 different splitting and cross-validation strategies, including different types of test datasets (e.g. non-harmonized vs. ComBat-harmonized datasets). The sensitivity, specificity, and area under the receiver operating characteristic (ROC) curve (AUC) were reported for models evaluation.

Results: In the test dataset (4301) consisting of CT and/or RT-PCR positive cases, AUC, sensitivity, and specificity of $0.83 \pm 0.01$ (CI95\%: 0.81-0.85), 0.81, and 0.72 , respectively, were obtained by ANOVA feature selector + RF classifier. In RT-PCR-only positive test sets (3644), similar results were achieved, and there was no statistically significant difference. In ComBat harmonized dataset, Relief feature selector + RF classifier resulted in highest performance of AUC, reaching $0.83 \pm 0.01$ (CI95\%: 0.81-0.85), with sensitivity and specificity of 0.77 and 0.74 , respectively. At the same time, ComBat harmonization did not depict statistically significant improvement relevant to non-harmonized dataset. In leave-one-center-out, the combination of ANOVA feature selector and LR classifier resulted in the highest performance of AUC $(0.80 \pm 0.084)$ with sensitivity and specificity of $0.77 \pm 0.11$ and $0.76 \pm 0.075$, respectively.

Conclusion: Lung CT radiomics features can be used towards robust prognostic modeling of COVID-19 in large heterogeneous datasets gathered from multiple centers. As such, CT radiomics-based model has significant potential for use in prospective clinical settings towards improved management of COVID-19 patients.

Key Words: X-ray CT, COVID-19, radiomics, prognosis, machine learning. 


\section{INTRODUCTION}

The novel coronavirus disease which emerged in 2019 (COVID-19) is now a major cause of death worldwide ${ }^{1}$. This highly contagious virus can cause a spectrum of pulmonary, hematological, neurological, and systemic complications, making it a highly lethal pathogen ${ }^{2}$. As of August $23^{\text {st }}, 2021$, there have been $>200$ million globally confirmed cases of COVID-19, including >4 million deaths and >4 billion vaccinations reported to the world health organization (WHO) [https://covid19.who.int/]. There remains an urgent need for addressing issues such as diagnosis, prognosis, and treatment options ${ }^{3}$.

Diagnostic tools for COVID-19, such as reverse transcription polymerase chain reaction (RT-PCR) aid to distinguish between negative and positive cases ${ }^{4}$. Prognostic tools, on the other hand, provide clinicians with insights to optimize treatment strategies, manage the hospitalization of patients both in the wards and intensive care units (ICU), and better handle patient follow-up plans ${ }^{5}$. Different studies have evaluated clinical and/or non-clinical features for determining the diagnosis and prognosis of patients with COVID-19. Yan et al. ${ }^{6}$ used only clinical features to classify patients into different categories, ranging from mild to critical conditions. Zhou et al. ${ }^{7}$ also aimed at establishing a prognostic model for outcome prediction of patients with COVID-19, utilizing their clinical data.

Computed Tomography (CT) plays a pivotal role in the management of a wide variety of diseases as a fast and non-invasive imaging modality. In the case of COVID-19, CT is used for both diagnostic (e.g. in case of limited access to RT-PCR) and prognostic purposes ${ }^{8}$. The clinical value of CT imaging relies mainly on the early detection of lung infections and high accuracy in quantifying the disease progression and severity ${ }^{9}$. Francone et al. ${ }^{10}$ assessed the correlation of CT scores with COVID-19 pneumonia severity and outcome. In another study, Zhao et al. ${ }^{11}$ specified pulmonary involvement severity by measuring the extent of pneumonia and consolidation that appear on CT images. Li et al. ${ }^{12}$ concluded that high scores of CT images are associated with severe COVID-19 pneumonia.

In spite of previously conducted research, there is still a need for studies with more accurate and comprehensive analyses ${ }^{13}$. In conventional analyses, CT features are visually and subjectively defined, while machine learning (ML) and/or deep learning (DL) models have the potential to provide more comprehensive and objective assessment of images. Towards modeling of outcomes in COVID-19 patients, several ML and DL algorithms have been utilized to assess severity and to predict outcome of patients using CT imaging ${ }^{14-17}$. To evaluate the sensitivity and specificity of CT for COVID-19 diagnostic purposes, Harmon et al. ${ }^{14}$ achieved 
a sensitivity of $84 \%$ and specificity of $93 \%$ in an independent test set of 1337 patients applying DL on CT images. In another machine learning study, Mei et al. ${ }^{15}$ reported a sensitivity of $84.3 \%$ and specificity of $82.8 \%$ based on combined CT and clinical data. Cai et al. ${ }^{16}$ utilized a random forest model to assess the severity of COVID-19 disease in 99 patients and their need for a longer hospital or ICU stay. Another study by Lessman et al. ${ }^{17}$ reported the model performance of three DL models for severity assessment in COVID-19. In another multi-center study, Meng et al. ${ }^{18}$ differentiated patients with high-risk of mortality versus low-risk ones using a convolutional neural network named De-COVID-Net. Ning et al. ${ }^{19}$ used their pretrained DL model on a dataset consisting of 351 patients that was capable of distinguishing between non-coronavirus pneumonia, mild coronavirus pneumonia, and severe forms of COVID-19 disease.

Various studies also reported remarkable prediction accuracies utilizing radiomics approaches using CT and chest $\mathrm{x}$-ray imaging modalities ${ }^{20-23}$. Medical images could be converted into high-dimensional data by means of radiomics, wherein radiomics features are selected from the images and combined using machine learning algorithms to arrive at radiomics signatures as biomarkers of disease ${ }^{24-34}$. In addition to wide usage in several oncologic ${ }^{35-37}$ as well as non-oncologic diseases ${ }^{27,38}$, radiomics studies have indicated that imaging features extracted from CT or chest X-ray images could be used as parameters for outcome prediction of patients with COVID-19 pneumonia. Radiomics analyses have been applied to different aspects of COVID-19, including diagnosis, severity scoring, prognosis, hospital/ICU stay prediction, and survival analysis ${ }^{20-23}$. In a retrospective study, Fu et al. ${ }^{39}$ constructed a predictive model based on CT radiomics, clinical and laboratory features. This signature could classify COVID-19 patients into stable and unstable (i.e. progressive phenotype). Homayounieh et al. ${ }^{40}$ aimed to predict the severity of pneumonia in patients with COVID-19 using a radiomics model that outperformed models consisting of clinical-only features. Another study by Li et al. 41 analyzed a radiomics/DL model that distinguished severe from critical COVID-19 pneumonia patients. Cai et al. ${ }^{42}$ developed a model by means of combining CT radiomics features and clinical data to predict RT-PCR negativity during admission. Yue et al. ${ }^{43}$ conducted a multicentric radiomics study on 52 patients to differentiate whether an individual needs a short-term or long-term hospital stay. Another study by Bae et al. ${ }^{44}$ predicted the mortality of patients with COVID-19 using chest x-ray radiomics. Their model could identify whether a patient needs mechanical ventilation or not.

Artificial intelligence (AI) has been widely used in radiology to provide diagnostic and prognostic tools to help clinicians during the pandemic. However, owing to the lack of 
1 standardization in AI studies in terms of data collection, methodology, and evaluation, most of 2 these studies were not pragmatic when it comes to clinical adoption ${ }^{13,45,46}$. In a recent study by

3 Roberts et al. ${ }^{13}$, possible sources of bias in more than 2000 AI articles in COVID-19 were evaluated in both deep learning and traditional machine learning-based studies. This review showed that bias do exist in most, if not all, of the studies in different domains, including dataset and methodology. In the dataset domain, several articles used public datasets which can contain duplicates, low-quality images, false demographics, or unknown clinical/lab data of patients. These public datasets can also induce bias in the outcome domain as they may fail to supply sufficient information about how they exactly proved a patient is COVID-19 positive or how imaging data were acquired in terms of image acquisition and reconstruction. They also mentioned using small datasets, Frankenstein datasets ${ }^{13}$, and Toy datasets ${ }^{45}$ in several articles. In the methodology domain, most of the studies did not provide all methodological information or did not perform a standard AI analysis based on guidelines ${ }^{47,48}$.

Overall, Roberts et al. ${ }^{13}$ reviewed 69 traditional machine learning/radiomics studies and reported that 44 were excluded because of Radiomics Quality Score (RQS) ${ }^{47}$ of less than six or not describing the datasets appropriately. From the remaining 25, six articles performed model evaluation using external validation sets and only four papers reported the significance of their model along with the statistical parameters (agreement level). They also assessed bias in the prognostication studies in the four areas of prediction model risk of bias assessment tool (PROBAST) guide and reported high bias in participants, predictors, outcomes, and analysis areas. Overall, there are several radiomics studies targeting improved COVID-19 diagnosis or prognosis. However, owing to the limited sample size, single-centered nature of most of the databases, and variability in data acquisition and image reconstruction parameters, the models tend to overfit ${ }^{13}$. Providing a generalizable model which is reproducible on unseen datasets of other centers is highly desired. In this context, we designed a large multi-institutional study to build and evaluate a radiomics model based on a large-scale CT imaging dataset aimed at the prediction of survival (alive or deceased) in COVID-19 infected patients. We built and evaluated our model based on different guidelines and tested different machine learning algorithms in different strategies to evaluate model reproducibility and repeatability in a large dataset. 
Figure 1 summarizes the different steps adopted in this study. To provide a standard and reproducible study, we completed different checklists/guidelines concerning predictive modeling, radiomics studies, and artificial intelligence studies. The Transparent Reporting of a multivariable prediction model for Individual Prognosis or Diagnosis (TRIPOD) [40] checklist is provided in supplemental Table 1. We also reported the Radiomics Quality Score (RQS) based on Lambin et al. ${ }^{47}$ and the Checklist for Artificial Intelligence in Medical imaging (CLAIM) ${ }^{48}$ in supplemental material. These checklists were filled out by two individuals (with consensus) who are experts in radiomics field and not co-authors in this study.

\section{Patient Population}

This study was approved by our local institutional review board (IRB), and written informed consent of patients was waived by the ethics committee as anonymized data were used without any interventional effect on diagnosis, treatment or management of COVID-19 patients.

In the first step, 24,448 patients, from 19 medical centers in Iran, suspected of COVID-19 and with acquired chest CT images were included. Different exclusion criteria were applied to provide a reliable dataset. We excluded: (i) patients without follow-up information or clear evidence of clinical endpoint, or if they were transferred to another medical center (3519 patients), or patients with (ii) negative RT-PCR (1860 patients), (iii) laboratory-confirmed pneumonia of other types (1606 patients), (iv) confirmed lung cancer or metastases from other origins to the lungs (1400 patients), (v) atypical CT findings for other abnormalities (850 patients), (vi) CT images with contrast media administration (58 patients), (vii) severe motion or bulk motion artifacts in CT images were carefully checked (515 patients), (viii) extremely inappropriate positioning which resulted in missing the upper and lower bounds of the lungs (121 patients), or (ix) CT images with extremely low quality or SNR (210 patients).

Considering these criteria, we excluded 10,149 patients from further analysis (Figure 2). Hence, 14,339 chest CT scans (with one scan per patient whose COVID-19 was confirmed either by RTPCR or CT imaging) were included in this study. Common symptoms of COVID-19, including fever, respiratory symptoms, shortness of breath, dry cough and tiredness were recorded and contact history with COVID-19 patients was also assessed. In each center, CT images were 
to settle the disagreement. As defined in the COVID-19 Reporting and Data System (CO-RADS)

$2{ }^{49}$, typical manifestations of COVID-19, such as ground-glass opacity, consolidation, crazy-paving pattern, or dominant peripheral distribution of parenchymal abnormalities were considered 4 diagnostic for COVID-19 in CT images.

5 Among these studies, 13,741 CT images were collected from 18 centers in Iran (1560 deceased, 12,171 alive; fraction of deceased cases are significantly overrepresented due to our exclusion criteria), and 608 images were gathered from an online open-access databases from China (Center 9: 18 deceased, 590 alive) ${ }^{50}$. All patients from Iran received standard treatment regimens according to the interim national COVID-19 treatment guideline [corona.behdasht.gov.ir]. Only one center (Center 10) included outpatient studies and the rest were inpatient-only studies from hospitalized patients. Follow-up was performed 3-4 months after the initial CT scan in the outpatient cases. For admitted patients (inpatient), follow-up was performed until discharge from the hospital, which was considered after careful evaluation of patients by the attending physician based on several criteria, including stable hemodynamics state (BP>90/60, HR<120), absence of fever for $>2$ days, absence of any respiratory distress, blood oxygen saturation $>93 \%$ in ambient

\section{CT Image Acquisition}

All chest $\mathrm{CT}$ images from the Iranian centers were acquired according to an institutional variation of the national society of radiology COVID-19 imaging guidelines ${ }^{51}$. Image acquisition was performed during breath-hold to reduce motion artifacts. Variations in CT imaging protocols among centers were observed which led to considerable variability in image quality and radiation dose. Volumetric CT Dose Index $\left(\mathrm{CTDI}_{\mathrm{vol}}\right)$, as a parameter representing vendor-free information on radiation exposure, was reported to better reflect intra/inter institutional variability of our dataset. Table 1 summarizes the image acquisition characteristics of each center, including the number of images, acquisition parameters (slice thickness, tube current), and CTDI ${ }_{\mathrm{vol}}$.

\section{Image Segmentation and Image Preprocessing}

The lungs were automatically segmented using our DL-based algorithm named COLI-NET which we previously proposed and evaluated ${ }^{52}$. For efficient radiomics feature extraction (feature 
obtain a computationally efficient feature extraction. After reviewing the segmentations, the image

2 voxel was resized to an isotropic voxel size of $1 \times 1 \times 1 \mathrm{~mm}^{3}$, and the intensity discretized to 643 binning size ${ }^{53}$.

\section{$4 \quad$ Radiomics Feature Extraction and Harmonization}

5 After image preprocessing, radiomics feature extraction was performed using the PyRadiomics

6 Python library ${ }^{54}$. Radiomics features, including morphological $(\mathrm{n}=16)$, intensity $(\mathrm{n}=17)$, and

7 texture features including second-order features, such as Gray Level Co-occurrence Matrix

8 (GLCM, n=24), higher-order features namely Gray Level Size Zone Matrix (GLSZM, n=16),

9 Neighboring Gray Tone Difference Matrix (NGTDM, n=5), Gray Level Run Length Matrix 10 (GLRLM, n=16), and Gray Level Dependence Matrix (GLDM, n=14) were extracted in 11 compliance with the Image Biomarker Standardization Initiative (IBSI) guidelines ${ }^{53}$.

\section{Feature Preprocessing}

13 For each feature vector, the mean and standard deviation were calculated (in training sets) and then normalized using Z-Score normalization, which consists of subtracting each feature vector from

\section{Feature Selection and Classification}

22 In this study, we used 4 feature selection algorithms, including Analysis of Variance (ANOVA), 23 Kruskal-Wallis (KW), Recursive Feature Elimination (RFE), and Relief. Feature preprocessing 24 and selection were performed on training sets and then applied on test sets. All test and external 25 validation sets were unseen to feature processing and the selection and model building process. 26 For classification task, we used seven classifiers, including Logistic Regression (LR), Least 27 Absolute Shrinkage and Selection Operator (LASSO), Linear Discriminant Analysis (LDA), 28 Random Forest (RF), AdaBoost (AB), Naïve Bayes (NB) and Multilayer Perceptron (MLP). By 
cross-combination of four feature selectors and seven classifiers, we tested twenty-eight different combinations.

\section{Evaluation}

For thorough assessment, we trained and evaluated our models using 10 different strategies as summarized in Figure 3. To evaluate the models on whole datasets without considering data variability in each center, we divided the dataset of each center to $70 \%$ training and $30 \%$ tests sets resulting in the following two strategies (1 and 2):

- $\quad$ Random Splitting method \#1: Non-harmonized datasets were randomly split into 70\% (10,038 patients) and 30\% (4301 patients) for training and test sets, respectively, without considering centers. The data included patients whose COVID-19 was confirmed using RT-PCR and patients confirmed only by imaging. This test dataset included both populations.

- Random Splitting method \#2: Non-harmonized datasets were randomly split into $70 \%$ (8503 patients) and 30\% (3644 patients) for the training and test sets, respectively, without considering centers. The train and test sets consisted of only patients with positive RT-PCR.

- To evaluate the models on whole datasets considering data variability in each center, we divided the dataset of each center to $70 \%$ training and $30 \%$ test sets resulting in the following two strategies (3 and 4):

- Random Splitting method \#3: Data from each center (non-harmonized) were randomly split into $70 \%$ (10,048 patients) and 30\% (4291 patients) for the training and test sets, respectively. As our data included patients whose COVID-19 was confirmed using RT-PCR and patients confirmed only by imaging, this test dataset included both populations.

- Random Splitting method \#4: Data from each center were randomly split into 70\% (10,704 patients) and 30\% (3635 patients) for the training and test sets, respectively. The train and test sets consist of only patients with positive RT-PCR.

To evaluate the models in the whole dataset by removing data variability due to acquisition/reconstruction from different centers, ComBat harmonization proposed by Johnson et al. ${ }^{55}$ was applied to the extracted features to tackle the effect of center-based imaging variability. The impact of ComBat harmonization on radiomics features was assessed by Kruskal-Wallis test. After applying ComBat harmonization, we divided the datasets of each center to $70 / 30 \%$ train/test sets resulting in the following two strategies (3 and 4): 
- Random Splitting method \#5: Data from each center (ComBat harmonization) were randomly split into $70 \%$ (10,048 patients) and 30\% (4291 patients) for the training and test sets, respectively. As our data included patients whose COVID-19 was confirmed using RT-PCR and patients confirmed only by imaging, this test dataset included both populations.

- Random Splitting method \#6: Data from each center (ComBat harmonization) were randomly split into 70\% (10,704 patients) and 30\% (3635 patients) for the training and test sets, respectively. The train and test sets consisted of only patients with positive RT-PCR.

To evaluate model generalizability and sensitivity to datasets, we performed model assessment using the following strategies (7 to 9 ) on the external validation sets:

- Random Splitting method \#7: Data (non-harmonized) were randomly split into 70\% (10,655 patients) and 30\% (3684 patients) for the training and external validation sets, respectively. The center's number in the test set appears in the test sets.

- Center-based model evaluation \#8: we built models on one center's dataset (non-harmonized) and then evaluated on 18 remaining centers (external validation set), and then repeated this process for all datasets.

- Leave-one-center-out (LOCO) \#9: On each of the 19 iterations, 18 centers were used as the training set, and one as the external validation set (unseen data during training). We repeated this process for all center datasets (non-harmonized).

To evaluate of model sensitivity to each dataset, we trained and tested the models in each center separately on each center dataset using the following strategies:

- Random Splitting method \#10: Data from each center (non-harmonized) were randomly split into $70 \%$ and $30 \%$ for the training and test sets, respectively. The models were built and evaluated on each center separately.

All multivariate steps, including feature preprocessing, feature selection and classification were performed separately for each strategy. Classification algorithms were optimized during training using grid search algorithms. The best models were chosen by one standard deviation rule in 10fold cross-validation and then evaluated on test or external validation sets. The accuracy, sensitivity, specificity, and area under the receiver operating characteristic curve (AUC) were reported for the test or external validation sets (unseen during training). Statistical comparison of 
1 AUCs (by 10000 bootstrapping) between models was performed using the DeLong test ${ }^{56}$. The 2 significance level was considered at a level of 0.05 . All multivariate analysis steps were performed 3 using Python Scikit-Learn open-source library.

4

5

6

7

8

9

10

11

12

13

14

15

16

17

18

19

20

21

22

23

24 


\section{RESULTS}

2 Figures 4 depicts the hierarchical clustering heat map of radiomics features distribution in alive and deceased groups for the whole dataset prior to ComBat harmonization. Supplemental Figure 1 shows the cluster heat map of radiomics features in the non-harmonized data set. Figure 5 shows the correlation of radiomics features in the whole dataset, whereas supplemental Figure 2 represents the same for ComBat harmonized features. The statistical differences calculated using the Kruskal-Wallis test are presented in supplemental Table 1 before and after ComBat harmonization. The results of ComBat harmonization showed that the algorithm properly eliminated the center effect on radiomics features in most features. ComBat harmonization data only were used for strategies 5 and 6 . Figures 6-8 provide the classifications power indices of AUC, sensitivity and specificity, respectively, for splitting strategies 1-10. More detailed results were presented in supplemental Tables 2-11 for the different strategies.

In strategy 1 where the data were randomly split into train and test sets (without considering centers), RFE feature selection and RF classifier results in highest performance of AUC $0.84 \pm 0.01$ (CI95\%: 0.82-0.85) with sensitivity and specificity of 0.78 and 0.76 , respectively. In strategy 2 where only PCR positive studies were randomly split into train and test sets (without considering centers), KW feature selection and RF classifier combination resulted in the highest performance with an AUC of 0.84 \pm 0.01 (CI95\%: 0.82-0.86) and sensitivity and specificity of 0.81 and 0.76 , respectively. There was no statistical significant difference between Strategies 1 and 2, the main difference being the inclusion of CT and PCR positive studies in strategy 1 and only PCR positive studies in strategy 2.

In strategy 3 where whole data splitting was performed in each center separately for train and test sets, ANOVA feature selector and RF classifier combination resulted in the highest performance with AUC of 0.83 \pm 0.01 (CI95\%: 0.81-0.85), sensitivity and specificity of 0.81 and 0.72, respectively. Similar results as above were achieved for strategy, 4 where data splitting was performed in each center separately to train and test set for PCR positive dataset. There were no statistically significant difference between strategies 3 and 4 where the main difference was including CT and PCR positive in strategy 4 and only PCR positive in strategy 5.

In strategy 5 where Combat harmonized whole data splitting was performed in each center separately to the train and test sets, Relief feature selector and RF classifier combination resulted 
specificity of 0.77 and 0.74 , respectively. In strategy 6 , where Combat harmonized data splitting was performed in each center separately to the train and test sets for PCR positive studies, Relief feature selector and RF classifier combination resulted in the highest performance with an AUC $0.83 \pm 0.01$ (CI95\%: $0.81-0.84$ ), sensitivity and specificity of 0.79 and 0.72 , respectively. . There 5 were no statistically significant differences between strategies 5 and 6 . The statistical comparison of AUCs between for ComBat harmonization strategies 5 and 6 and to the same splitting in strategies 3 and 4 using DeLong test didn't reveal any statistically significant differences.

In strategy 7, where the splitting into train and test sets was performed based on centers (centers appear in training and test sets only once), RFE selector and RF classifier combination resulted in the highest performance with an AUC of 0.79 \pm 0.01 (CI95\%: 0.76-0.81), sensitivity and specificity of 0.73 and 0.71, respectively. In Figure 9, the ROC curves of the test set for strategies 1-7 as well as the comparison of the different strategies are depicted. In strategy 8 where the model is built based on one center dataset (non-harmonized) and then evaluated on the 18 remaining centers (external validation set), ANOVA feature selector and NB classifier combination resulted in the highest performance with an AUC of $0.74 \pm 0.034$, sensitivity and specificity of $0.71 \pm 0.026$ and $0.69 \pm 0.033$, respectively. The results of each center were presented in supplemental Table 12 . Tables 13 and 14 for strategies 9 and 10, respectively. 


\section{DISCUSSION}

In this multi-centric study, we conducted a CT-based radiomics analysis to assess the ability of our model in predicting the overall survival of patients with COVID-19 using a large multiinstitutional dataset. We included 14,339 patients along with their CT images, segmented the lungs, and extracted distinct radiomics features. We evaluated different combinations of feature selectors and classifiers in different strategies. Since the dataset was gathered from different centers, we applied the ComBat Harmonization algorithm that has been successfully applied in radiomics studies over the extracted features ${ }^{57}$. As our dataset consisted of imbalanced classes, we first used SMOT algorithm in the training sets. Our model was trained and the results of 3 different testing methods were reported.

Prognostic modeling can be regarded as an important framework towards better understanding of disease, its management, monitoring, and identification of the best treatment options. A number of reports have shown the effectiveness of image-based, laboratory-based, or combined models in outcome prediction of COVID-19 infected patients ${ }^{58,59}$. Qiu et al. ${ }^{60}$ constructed a radiomics model trained to classify the severity of COVID-19 lesions (mild vs severe) using CT images. Their study included a medium-to-large number of patients $(n=1160)$ and achieved an AUC of 0.87 in the test dataset. They showed that the radiomics signature is potent in aiding physicians to manage patients in a more precise way. Fu et al. ${ }^{39}$ conducted a similar experiment with a radiomics-based model using CT images and applied it to data from 64 patients to classify them into progressive and stable groups. Their model could accurately perform the given task (AUC=0.83). While the results were promising, their study did not include a large cohort.

A study by Chao et al. ${ }^{59}$ included different types of information, such as CT-based radiomics features, clinical, and demographic data to employ a holistic prognostic model. Their model could predict whether the patients will demand an ICU admission or not with an AUC of 0.88. Tang et al. ${ }^{61}$ also assessed a random forest model for classifying patients into categories of severe and non-severe based on CT imaging radiomics features along with laboratory test results. The model performed well (AUC $=0.98$ ) on their dataset consisting of 118 patients. In a study by Wu et al. ${ }^{62}$, the authors assessed the predictive power of a radiomic signature for showing poor patient outcomes defined as ICU admission, need for mechanical ventilation, or death. Their model could reach an AUC of 0.97 in the prediction of 28-day outcomes after CT images were taken. This highly promising result was achieved with the help of clinical data and harmonization of the 
features. At the same time, in our study, ComBat harmonization did not appear to impact outcome prediction.

One should note that both clinical-only and radiomics-only survival prediction models have advantages. However, studies have shown that radiomics features yield superior accuracy in most cases. In a study by Homayounieh et al. ${ }^{63}$, the authors developed a radiomics-based signature and compared it with a clinical-only signature in terms of mortality prediction. They concluded that radiomics-based model can outperform the clinical-only model with a wide margin (AUC of 0.81 versus 0.68 ). Their study included 315 adults and was applied to other clinical outcomes as well, such as the prediction of outpatient/inpatient care and ICU admission. In addition, other reports indicated that adding clinical features to the radiomics model only slightly improved the results ${ }^{64}$. In a recent study, Shiri et al. ${ }^{64}$ performed a radiomics study for prognostication purpose (alive or deceased) of COVID-19 patients using clinical (demographic, laboratory, and radiological scoring), COVID-19 pneumonia lesion radiomics features and whole lung radiomics features, separately and in combination. They trained a machine learning algorithm, Maximum Relevance Minimum Redundancy (MRMR) as the feature selector and XGBoost as the classifier, on 106 patients and evaluated and reported results on 46 test sets. They reported an AUC of $0.87 \pm 0.04$ for clinical-only, $0.92 \pm 0.03$ for whole lung radiomics, $0.92 \pm 0.03$ for lesion radiomics, $0.91 \pm$ 0.04 for lung + lesion radiomics, $0.92 \pm 0.03$ for lung radiomics + clinical data, $0.94 \pm 0.03$ for lesion radiomics + clinical data and $0.95 \pm 0.03$ for lung + lesion radiomics + clinical data. The lung and lesion radiomics-only models showed similar performance, while the integration of features resulted in the highest accuracy.

Lassau et al. ${ }^{65}$ combined CT-based DL models, biological and clinical features for severity prediction in 1003 COVID-19 patients, confirmed by either CT or RT-PCR. They showed clinical and biological features correlation with CT markers. Zhang et al. ${ }^{58}$ conducted a diagnostic and prognostic study using 3777 COVID-19 patients. They reported a high positive and negative correlation of lung-lesion CT manifestations with a number of clinical and laboratory tests. They also reported that their diagnostic model (COVID-19 from common pneumonia and normal control) can improve radiologist's performance from junior to senior level (AUC $=0.98$ ) for progression to severe/critical disease in their prognostic model. They reported an AUC of 0.90 with sensitivity and specificity of 0.80 and 0.86 , respectively. Feng et al. ${ }^{66}$ built a machine learning prognostic model using a multicenter COVID-19 dataset. They reported a high correlation of CT 
features with clinical findings, also utilizing a multivariable model in the validation set consisting of 106 patients. The AUC was 0.89 (95\% CI: 0.81-0.98). Recently, Xu et al. ${ }^{67}$ conducted a multicentric study for the prediction of ICU admission, mechanical ventilation, and mortality of hospitalized patients with COVID-19. CT radiomics features were integrated with demographic and laboratory tests. The evaluation was performed in 1362 patients from nine hospitals reporting an AUC of $0.916,0.919$ and 0.853 for ICU admission, mechanical ventilation, and mortality of hospitalized patients, respectively. For the radiomics-only model, they reached an AUC of 0.86, 0.80 , and 0.66 for the above three mentioned outcomes, respectively.

Most previous studies suffered from a common limitation of COVID-19 RT-PCR not being available for the entire dataset when using multicentric data. In our study, COVID-19 positivity was confirmed by either RT-PCR or CT images, and different strategies were adopted to evaluate the models, including random splits and leave-one-center-out. We randomly split the data to train and test sets containing both CT positive and RT-PCR positive patients. Furthermore, to ensure the reproducibility of our results on RT-PCR positive patients, we split the dataset in a way that the test set consisted only of RT-PCR positive patients. To maximize the generalizability of the model and avoid overfitting on training sets, owing to variability in acquisition and reconstruction protocols, our model was developed on multicentric datasets with a wide variety of acquisition and reconstruction parameters. To test the generalizability of our model, we repeated the evaluation of our model using leave-one-center-out cross-validation. The results were reported for 10 different strategies of splitting and cross-validation scenarios.

Several studies reported on the use of CT radiomics or DL algorithms for diagnostic and prognostic purposes in patients with COVID-19 58,59,68. However, most studies were performed using a small sample size. Overall, establishing evidence that radiomics features can help prioritize patients based on the severity of their disease and/or predicting their survival requires assessment using larger cohorts for a more generalizable model because of the wide variability in COVID-19 manifestations in different patients. In this study, we provided a large multinational multicentric dataset and evaluated our model in different scenarios to ensure model reproducibility, robustness and generalizability.

While attempting to address bias and limitations to create a generalizable model, the results should be interpreted considering some issues. First, motion artifacts were unavoidable in some COVID-19 patient scans which resulted in overlapping pneumonia regions. We removed patients 
1 with severe motion artifacts to omit this effect on model generalization. Second, we enrolled 2 patients with common symptoms of COVID-19 whose infection was confirmed by either RT-PCR 3 or CT imaging (typical manifestation of COVID-19 defined by interim guidelines). We handled 4 this issue by testing different scenarios, including training a model using RT-PCR or CT positive 5 patients and held out only RT-PCR patients in the test set and reported reproducible and repeatable 6 results. Third, we did not include comorbidities (increased risk of adverse outcome), clinical or 7 laboratory data during modeling. However, previous studies showed high correlation of lung 8 features with these findings ${ }^{58,65,66}$. Future studies combining various information to build a holistic 9 model using a large dataset could improve the model's performance. Forth, we built a prognostic model based on all lung radiomics features. However, COVID-19 can result in imaging 11 manifestations in other organs, such as the heart. Including features from different organs has the 12 potential of improving prognostic performance ${ }^{69}$. Fifth, therapeutic regimens for different patients were not considered during modeling although providing this information may help improving the 14 accuracy of the model. Sixth, only binary classification was considered for the prognostic model 15 in this study. Future studies should perform survival analysis using time-to-event models to account for the time of adverse event. Lastly, we did not evaluate the impact of image acquisition

17 or reconstruction parameters on radiomics features at the same time. We applied ComBat 18 harmonization algorithm to eliminate center-specific parameter effects on CT radiomics features. 
2 A very large heterogeneous COVID-19 database was gathered from multiple centers and a 3 predictive model of survival outcome derived and extensively tested to evaluate its reproducibility 4 and generalizability. We demonstrated that lung CT radiomics features could be used as 5 biomarkers for prognostic modeling in COVID-19. Through the use of a large imaging dataset, the 6 predictive power of the proposed CT radiomics model is more reliable and may be prospectively 7 used in clinical setting to manage COVID-19 patients.

8

\section{ACKNOWLEDGMENTS}

10 This work was supported by the Swiss National Science Foundation under grant SNRF 11 320030_176052.

\section{Data and code availability}

14 Radiomics features and code would be available with request upon publication.

\section{Conflict of Interest statement}

17 The authors declare that they have no conflict of interest. 
1. Woolf, S.H., Chapman, D.A. \& Lee, J.H. COVID-19 as the Leading Cause of Death in the United States. JAMA 325, 123-124 (2021).

2. Lai, C.-C., Shih, T.-P., Ko, W.-C., Tang, H.-J. \& Hsueh, P.-R. Severe acute respiratory syndrome coronavirus 2 (SARS-CoV-2) and coronavirus disease-2019 (COVID-19): The epidemic and the challenges. Int J Antimicrob Agents 55, 105924-105924 (2020).

3. Lai, C.-C., Ko, W.-C., Lee, P.-I., Jean, S.-S. \& Hsueh, P.-R. Extra-respiratory manifestations of COVID-19. Int J Antimicrob Agents 56, 106024-106024 (2020).

4. Afzal, A. Molecular diagnostic technologies for COVID-19: Limitations and challenges. Journal of advanced research 26, 149-159 (2020).

5. Gill, T.M. The central role of prognosis in clinical decision making. JAMA 307, 199-200 (2012).

6. Yan, X., et al. Clinical Characteristics and Prognosis of 218 Patients With COVID-19: A Retrospective Study Based on Clinical Classification. Frontiers in medicine 7, 485-485 (2020).

7. Zhou, W., Qin, X., Hu, X., Lu, Y. \& Pan, J. Prognosis models for severe and critical COVID-19 based on the Charlson and Elixhauser comorbidity indices. International journal of medical sciences 17, 2257-2263 (2020).

8. Pontone, G., et al. Role of computed tomography in COVID-19. J Cardiovasc Comput Tomog (2020).

9. Yang, R., et al. Chest CT Severity Score: An Imaging Tool for Assessing Severe COVID-19. Radiology: Cardiothoracic Imaging 2, e200047 (2020).

10. Francone, M., et al. Chest CT score in COVID-19 patients: correlation with disease severity and short-term prognosis. Eur Radiol 30, 6808-6817 (2020).

11. Zhao, W., Zhong, Z., Xie, X., Yu, Q. \& Liu, J. Relation Between Chest CT Findings and Clinical Conditions of Coronavirus Disease (COVID-19) Pneumonia: A Multicenter Study. American Journal of Roentgenology 214, 1072-1077 (2020).

12. Li, K., et al. The Clinical and Chest CT Features Associated With Severe and Critical COVID-19 Pneumonia. Investigative radiology 55, 327-331 (2020).

13. Roberts, M., et al. Common pitfalls and recommendations for using machine learning to detect and prognosticate for COVID-19 using chest radiographs and CT scans. Nature Machine Intelligence 3, 199-217 (2021).

14. Harmon, S.A., et al. Artificial intelligence for the detection of COVID-19 pneumonia on chest CT using multinational datasets. Nature communications 11, 4080 (2020).

15. Mei, X., et al. Artificial intelligence-enabled rapid diagnosis of patients with COVID-19. Nat Med 26, 1224-1228 (2020).

16. Cai, W., et al. CT Quantification and Machine-learning Models for Assessment of Disease Severity and Prognosis of COVID-19 Patients. Academic radiology 27, 1665-1678 (2020).

17. Lessmann, N., et al. Automated Assessment of CO-RADS and Chest CT Severity Scores in Patients with Suspected COVID-19 Using Artificial Intelligence. Radiology, 202439 (2020).

18. Meng, L., et al. A Deep Learning Prognosis Model Help Alert for COVID-19 Patients at HighRisk of Death: A Multi-center Study. IEEE journal of biomedical and health informatics $\mathbf{P p}(2020)$.

19. Ning, W., et al. Open resource of clinical data from patients with pneumonia for the prediction of COVID-19 outcomes via deep learning. Nature biomedical engineering (2020).

20. Fang, M., et al. CT radiomics can help screen the coronavirus disease 2019 (COVID-19): a preliminary study. Science China Information Sciences 63, 1-8 (2020).

21. Wu, Q., et al. Radiomics Analysis of Computed Tomography helps predict poor prognostic outcome in COVID-19. Theranostics 10, 7231 (2020).

22. Homayounieh, F., et al. CT Radiomics, Radiologists and Clinical Information in Predicting Outcome of Patients with COVID-19 Pneumonia. Radiology: Cardiothoracic Imaging 2, e200322 (2020). 
23. Wang, H., et al. Decoding COVID-19 pneumonia: comparison of deep learning and radiomics CT image signatures. European journal of nuclear medicine and molecular imaging, 1-9 (2020).

24. Abdollahi, H., Shiri, I. \& Heydari, M. Medical Imaging Technologists in Radiomics Era: An Alice in Wonderland Problem. Iran J Public Health 48, 184-186 (2019).

25. Amini, M., et al. Multi-level multi-modality (PET and CT) fusion radiomics: prognostic modeling for non-small cell lung carcinoma. Phys Med Biol 66(2021).

26. Bouchareb, Y., et al. Artificial intelligence-driven assessment of radiological images for COVID19. Comput Biol Med 136, 104665 (2021).

27. Edalat-Javid, M., et al. Cardiac SPECT radiomic features repeatability and reproducibility: A multi-scanner phantom study. J Nucl Cardiol (2020).

28. Khodabakhshi, Z., et al. Overall Survival Prediction in Renal Cell Carcinoma Patients Using Computed Tomography Radiomic and Clinical Information. J Digit Imaging 34, 1086-1098 (2021).

29. Khodabakhshi, Z., et al. Non-small cell lung carcinoma histopathological subtype phenotyping using high-dimensional multinomial multiclass CT radiomics signature. Comput Biol Med 136, 104752 (2021).

30. Nazari, M., Shiri, I. \& Zaidi, H. Radiomics-based machine learning model to predict risk of death within 5-years in clear cell renal cell carcinoma patients. Comput Biol Med 129, 104135 (2021).

31. Shayesteh, S., et al. Treatment response prediction using MRI-based pre-, post-, and delta-radiomic features and machine learning algorithms in colorectal cancer. Med Phys 48, 3691-3701 (2021).

32. Shiri, I., Abdollahi, H., Shaysteh, S. \& Mahdavi, S.R. Test-retest reproducibility and robustness analysis of recurrent glioblastoma MRI radiomics texture features. Iranian Journal of Radiology (2017).

33. Shiri, I., et al. Machine learning-based prognostic modeling using clinical data and quantitative radiomic features from chest CT images in COVID-19 patients. Comput Biol Med 132, 104304 (2021).

34. Amini, M., et al. Overall Survival Prognostic Modelling of Non-small Cell Lung Cancer Patients Using Positron Emission Tomography/Computed Tomography Harmonised Radiomics Features: The Quest for the Optimal Machine Learning Algorithm. Clinical Oncology.

35. Mostafaei, S., et al. CT imaging markers to improve radiation toxicity prediction in prostate cancer radiotherapy by stacking regression algorithm. La radiologia medica 125, 87-97 (2020).

36. Nazari, M., Shiri, I. \& Zaidi, H. Radiomics-based machine learning model to predict risk of death within 5-years in clear cell renal cell carcinoma patients. Comput Biol Med 129, 104135 (2020).

37. Akhavanallaf, A., Shiri, I., Arabi, H. \& Zaidi, H. Whole-body voxel-based internal dosimetry using deep learning. Eur J Nucl Med Mol Imaging (2020).

38. Shiri, I., et al. Diagnosis of COVID-19 Using CT image Radiomics Features: A Comprehensive Machine Learning Study Involving 26,307 Patients. medRxiv (2021).

39. Fu, L., Li, Y., Cheng, A., Pang, P. \& Shu, Z. A Novel Machine Learning-derived Radiomic Signature of the Whole Lung Differentiates Stable From Progressive COVID-19 Infection: A Retrospective Cohort Study. Journal of thoracic imaging (2020).

40. Homayounieh, F., et al. Computed Tomography Radiomics Can Predict Disease Severity and Outcome in Coronavirus Disease 2019 Pneumonia. Journal of computer assisted tomography $\mathbf{4 4}$, 640-646 (2020).

41. Li, C., et al. Classification of Severe and Critical COVID-19 Using Deep Learning and Radiomics. IEEE journal of biomedical and health informatics $\mathbf{P p}(2020)$.

42. Cai, Q., et al. A model based on CT radiomic features for predicting RT-PCR becoming negative in coronavirus disease 2019 (COVID-19) patients. BMC medical imaging 20, 118 (2020).

43. Yue, H., et al. Machine learning-based CT radiomics method for predicting hospital stay in patients with pneumonia associated with SARS-CoV-2 infection: a multicenter study. Annals of translational medicine 8, 859 (2020).

44. Bae, J., et al. Predicting Mechanical Ventilation Requirement and Mortality in COVID-19 using Radiomics and Deep Learning on Chest Radiographs: A Multi-Institutional Study. ArXiv (2020). 
45. Tizhoosh, H.R. \& Fratesi, J. COVID-19, AI enthusiasts, and toy datasets: radiology without radiologists. Eur Radiol 31, 3553-3554 (2021).

46. Summers, R.M. Artificial Intelligence of COVID-19 Imaging: A Hammer in Search of a Nail. Radiology 298, E162-e164 (2021).

47. Lambin, P., et al. Radiomics: the bridge between medical imaging and personalized medicine. Nat Rev Clin Oncol 14, 749-762 (2017).

48. Mongan, J., Moy, L. \& Charles E. Kahn, J. Checklist for Artificial Intelligence in Medical Imaging (CLAIM): A Guide for Authors and Reviewers. Radiology: Artificial Intelligence 2, e200029 (2020).

49. Prokop, M., et al. CO-RADS: A Categorical CT Assessment Scheme for Patients Suspected of Having COVID-19-Definition and Evaluation. Radiology 296, E97-e104 (2020).

50. Ning, W., et al. Open resource of clinical data from patients with pneumonia for the prediction of COVID-19 outcomes via deep learning. Nat Biomed Eng 4, 1197-1207 (2020).

51. Radpour, A., et al. COVID-19 evaluation by low-dose high resolution CT scans protocol. Academic radiology 27, 901 (2020).

52. Shiri, I., et al. COLI-NET: Fully Automated COVID-19 Lung and Infection Pneumonia Lesion Detection and Segmentation from Chest CT Images. medRxiv, 2021.2004.2008.21255163 (2021).

53. Zwanenburg, A., et al. The image biomarker standardization initiative: standardized quantitative radiomics for high-throughput image-based phenotyping. Radiology 295, 328-338 (2020).

54. van Griethuysen, J.J.M., et al. Computational Radiomics System to Decode the Radiographic Phenotype. Cancer research 77, e104-e107 (2017).

55. Johnson, W.E., Li, C. \& Rabinovic, A. Adjusting batch effects in microarray expression data using empirical Bayes methods. Biostatistics 8, 118-127 (2006).

56. Robin, X., et al. pROC: an open-source package for R and $\mathrm{S}+$ to analyze and compare ROC curves. BMC bioinformatics 12, 1-8 (2011).

57. Da-Ano, R., Visvikis, D. \& Hatt, M. Harmonization strategies for multicenter radiomics investigations. Phys Med Biol 65, 24tr02 (2020).

58. Zhang, K., et al. Clinically Applicable AI System for Accurate Diagnosis, Quantitative Measurements, and Prognosis of COVID-19 Pneumonia Using Computed Tomography. Cell 181, 1423-1433.e1411 (2020).

59. Chao, H., et al. Integrative analysis for COVID-19 patient outcome prediction. Medical image analysis 67, 101844 (2020).

60. Qiu, J., et al. A Radiomics Signature to Quantitatively Analyze COVID-19-Infected Pulmonary Lesions. Interdisciplinary sciences, computational life sciences, 1-12 (2021).

61. Tang, Z., et al. Severity assessment of COVID-19 using CT image features and laboratory indices. Physics in medicine and biology (2020).

62. Wu, Q., et al. Radiomics Analysis of Computed Tomography helps predict poor prognostic outcome in COVID-19. Theranostics 10, 7231-7244 (2020).

63. Homayounieh, F., et al. CT Radiomics, Radiologists, and Clinical Information in Predicting Outcome of Patients with COVID-19 Pneumonia. Radiology: Cardiothoracic Imaging 2, e200322 (2020).

64. Shiri, I., et al. Machine Learning-based Prognostic Modeling using Clinical Data and Quantitative Radiomic Features from Chest CT Images in COVID-19 Patients. Computers in Biology and Medicine, 104304 (2021).

65. Lassau, N., et al. Integrating deep learning CT-scan model, biological and clinical variables to predict severity of COVID-19 patients. Nature communications 12, 1-11 (2021).

66. Feng, Z., et al. Early prediction of disease progression in COVID-19 pneumonia patients with chest CT and clinical characteristics. Nature communications 11, 4968 (2020).

67. $\mathrm{Xu}, \mathrm{Q}$., et al. CT-based Rapid Triage of COVID-19 Patients: Risk Prediction and Progression Estimation of ICU Admission, Mechanical Ventilation, and Death of Hospitalized Patients. medRxiv : the preprint server for health sciences (2020). 
1 68. Chassagnon, G., et al. AI-driven quantification, staging and outcome prediction of COVID-19

2 pneumonia. Medical image analysis 67, 101860 (2020).

3 69. Chassagnon, G., et al. AI-driven quantification, staging and outcome prediction of COVID-19 pneumonia. Medical image analysis 67, 101860 (2021). 
Table 1. Demographics and data acquisition parameters across different centers.

\begin{tabular}{|c|c|c|c|c|c|c|c|c|}
\hline \multirow{2}{*}{ Center } & \multirow{2}{*}{ Number (Deceased \%) } & \multirow{2}{*}{$\begin{array}{c}\text { PCR } \\
\% \text { of patients with available PCR result }\end{array}$} & \multicolumn{2}{|c|}{ Gender } & \multirow{2}{*}{$\begin{array}{c}\text { CTDI }_{\text {vol }} \\
\text { Mean } \pm \text { SD }\end{array}$} & \multirow{2}{*}{$\begin{array}{l}\text { Tube Current } \\
\text { Mean } \pm \text { SD }\end{array}$} & \multirow{2}{*}{$\begin{array}{c}\text { Age } \\
\text { Mean } \pm \text { SD }\end{array}$} & \multirow{2}{*}{$\begin{array}{l}\text { Slice Thickness } \\
\qquad \text { Mean } \pm \text { SD }\end{array}$} \\
\hline & & & Male & Female & & & & \\
\hline Center_01 & $152(26.3 \%)$ & $100 \%$ & 87 & 65 & $1.57 \pm 0.32$ & $45 \pm 47.8$ & $62.1 \pm 17.0$ & $1.50 \pm 0.50$ \\
\hline Center_02 & $264(41.6 \%)$ & $99 \%$ & 120 & 144 & $8.47 \pm 0.53$ & $186.2 \pm 34.0$ & $71.8 \pm 12.4$ & $3.44 \pm 0.95$ \\
\hline Center_03 & $319(1.5 \%)$ & $100 \%$ & 168 & 151 & $8.55 \pm 4.61$ & $185 \pm 65.3$ & $51.6 \pm 17.9$ & $2.82 \pm 0.99$ \\
\hline Center_04 & $373(22.5 \%)$ & $77 \%$ & 157 & 216 & $7.52 \pm 1.23$ & $147.1 \pm 43.2$ & $54.1 \pm 19.3$ & $5.22 \pm 0.46$ \\
\hline Center_05 & $382(19.1 \%)$ & $100 \%$ & 215 & 167 & $6.37 \pm 0.67$ & $128.3 \pm 4.5$ & $58.2 \pm 18.25$ & $7.67 \pm 1.7$ \\
\hline Center_06 & $394(38.6 \%)$ & $100 \%$ & 257 & 137 & $5.8 \pm 2.52$ & $149 \pm 48.3$ & $56.8 \pm 16.8$ & $2.34 \pm 0.24$ \\
\hline Center_07 & $492(5.2 \%)$ & $100 \%$ & 276 & 216 & $9.68 \pm 2.91$ & $349 \pm 181$ & $33.0 \pm 5.66$ & $2.57 \pm 1.36$ \\
\hline Center_08 & $539(19.1 \%)$ & $94 \%$ & 287 & 252 & $4.10 \pm 4.57$ & $167 \pm 39.1$ & $54.1 \pm 17.4$ & $7.71 \pm 0.77$ \\
\hline Center_09 & $608(3.0 \%)$ & $100 \%$ & 274 & 334 & $6.61 \pm 4.87$ & $164 \pm 128$ & $55.2+15.99$ & $2.10 \pm 1.21$ \\
\hline Center_10 & $685(14.8 \%)$ & $89 \%$ & 348 & 337 & $5.01 \pm 3.57$ & $171 \pm 33.3$ & $56.4 \pm 18.7$ & $7.16 \pm 0.81$ \\
\hline Center_11 & $866(13.2 \%)$ & $100 \%$ & 520 & 346 & $3.40 \pm 0.42$ & $123.6 \pm 9.6$ & $55.4 \pm 17.9$ & $2.04 \pm 0.23$ \\
\hline Center_12 & $883(11.2 \%)$ & $62 \%$ & 448 & 435 & $4.30 \pm 1.54$ & $166.2 \pm 51.3$ & $55.4 \pm 15.6$ & $4.99 \pm 0.16$ \\
\hline Center_13 & $949(10.9 \%)$ & $76 \%$ & 568 & 381 & $5.57 \pm 1.21$ & $210.7 \pm 39.6$ & $52.6 \pm 19.3$ & $2.17 \pm 1.51$ \\
\hline Center_14 & $1022(9.7 \%)$ & $100 \%$ & 526 & 496 & $5.01 \pm 3.19$ & $91.6 \pm 48.6$ & $56.2 \pm 18.8$ & $4.93 \pm 0.49$ \\
\hline Center_15 & $1024(1.0 \%)$ & $29 \%$ & 542 & 482 & $6.82 \pm 1.02$ & $228.4 \pm 27.2$ & $48.3 \pm 14.5$ & $2.48 \pm 1.96$ \\
\hline Center_16 & $1180(12.7 \%)$ & $84 \%$ & 578 & 602 & $5.63 \pm 2.98$ & $172 \pm 34.1$ & $55.4 \pm 16.7$ & $6.38 \pm 0.82$ \\
\hline Center_17 & $1323(12.7 \%)$ & $100 \%$ & 680 & 643 & $6.14 \pm 1.41$ & $175 \pm 38.2$ & $57.4 \pm 18.7$ & $5.66 \pm 0.82$ \\
\hline Center_18 & $1355(2.5 \%)$ & $70 \%$ & 752 & 603 & $4.21 \pm 3.18$ & $98.4 \pm 41.1$ & $55.1 \pm 19.1$ & $4.75 \pm 0.69$ \\
\hline Center_19 & $1529(5.6 \%)$ & $93 \%$ & 814 & 715 & $8.92 \pm 1.40$ & $220.7 \pm 27.9$ & $54.5 \pm 19.0$ & $6.96 \pm 0.34$ \\
\hline
\end{tabular}


medRxiv preprint doi: https://doi.org/10.1101/2021.12.07.21267364; this version posted December 7, 2021. The copyright holder for this preprint (which was not certified by peer review) is the author/funder, who has granted medRxiv a license to display the preprint in

It is made available under a CC-BY-NC-ND 4.0 International license .
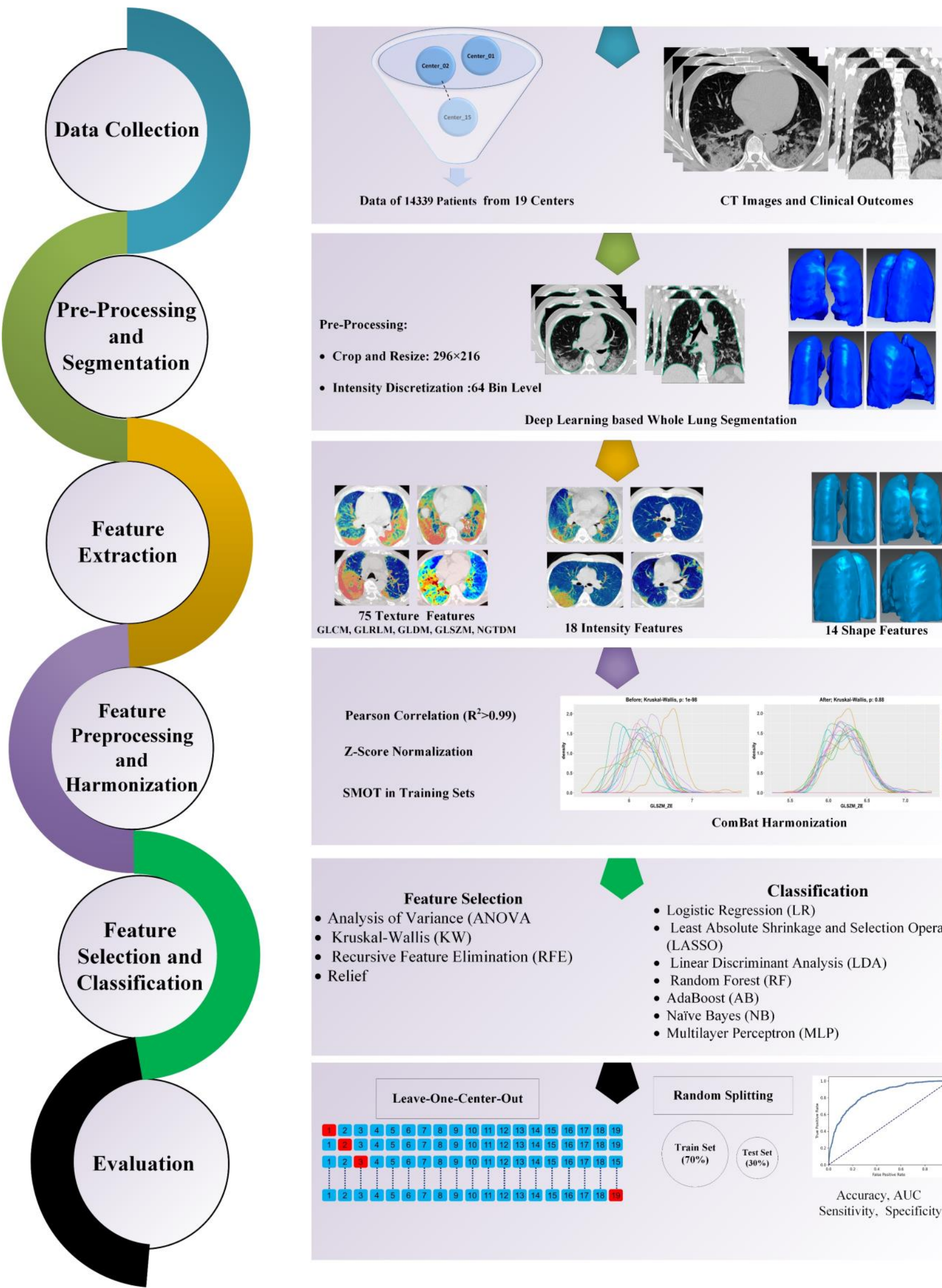

Deep Learning based Whole Lung Segmentation

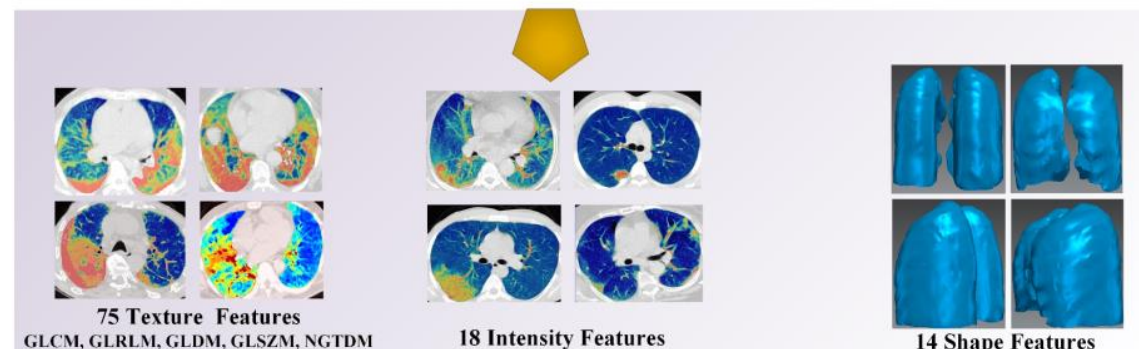

GLCM, GLRLM, GLDM, GLSZMI, NGTDM

18 Intensity Features

14 Shape Features
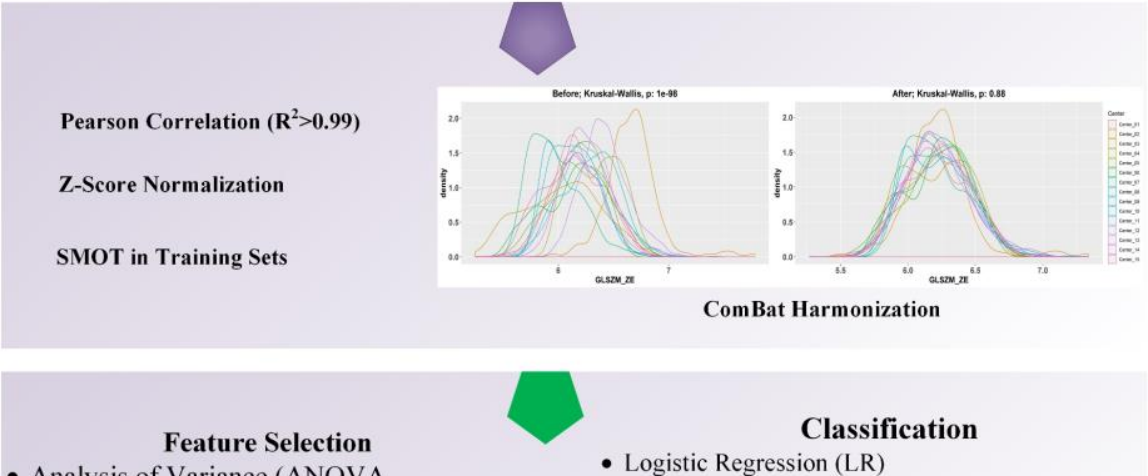

- Analysis of Variance (ANOVA

- Kruskal-Wallis (KW)

- Recursive Feature Elimination (RFE)

- Relief

$$
\text { Leave-One-Center-Out }
$$

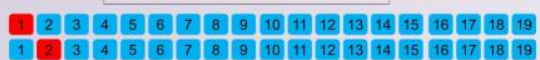
(4)

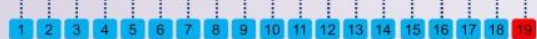

- Least Absolute Shrinkage and Selection Operator (LASSO)

- Linear Discriminant Analysis (LDA)

- Random Forest (RF)

- AdaBoost (AB)

- Naïve Bayes (NB)

- Multilayer Perceptron (MLP)

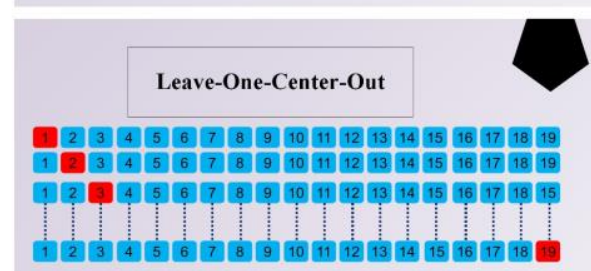

Random Splitting
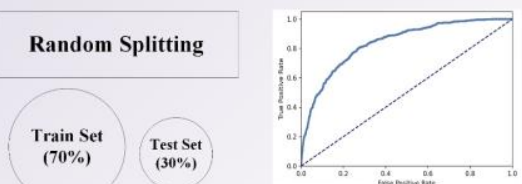

Accuracy, AUC Sensitivity, Specificity

Figure 1: The flow chart of our study represents the different radiomics steps. 


\section{Patients with CT images suspected for SARS-CoV2 infection from 15 Medical Centers}

\section{Exclusion criteria:}

- Patients without the follow-up information or without clear evidence of end point, or transferred to another medical center (3519 patients)

- Patients with negative RT-PCR (1860 patients)

- Patients with laboratory confirmed bacterial pneumonia (1606 patients)

- Patients with severe motion artifacts (515 patients)

- Patients with confirmed lung cancer or metastases in lung (1400 patients)

- Patients with atypical CT findings for other abnormalities (850 patients)

- Patient with extremely inappropriate positioning which result in missing the upper and lower bound of lung (121 patients)

- CT images with a extremely low quality or SNR (210 patients)

- CT Images with contrast media administration (58 patients)

\section{Patients SARS-CoV2 infection confirmed enrolled in this study}

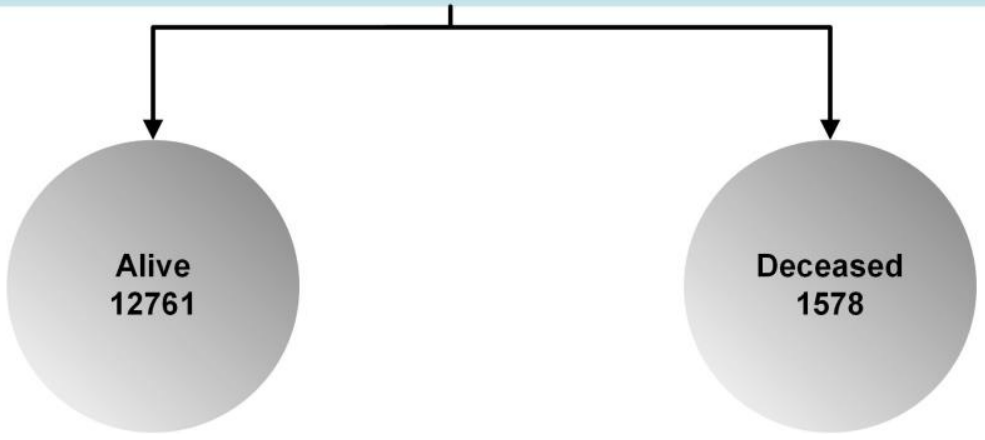

Figure 2: Inclusion and exclusion criteria in this study. Fraction of deceased patients is overrepresented in our data due to our exclusion criteria. 
medRxiv preprint doi: https://doi.org/10.1101/2021.12.07.21267364; this version posted December 7, 2021. The copyright holder for this preprint (which was not certified by peer review) is the author/funder, who has granted medRxiv a license to display the preprint in It is made available under a CC-BY-NC-ND 4.0 International license .
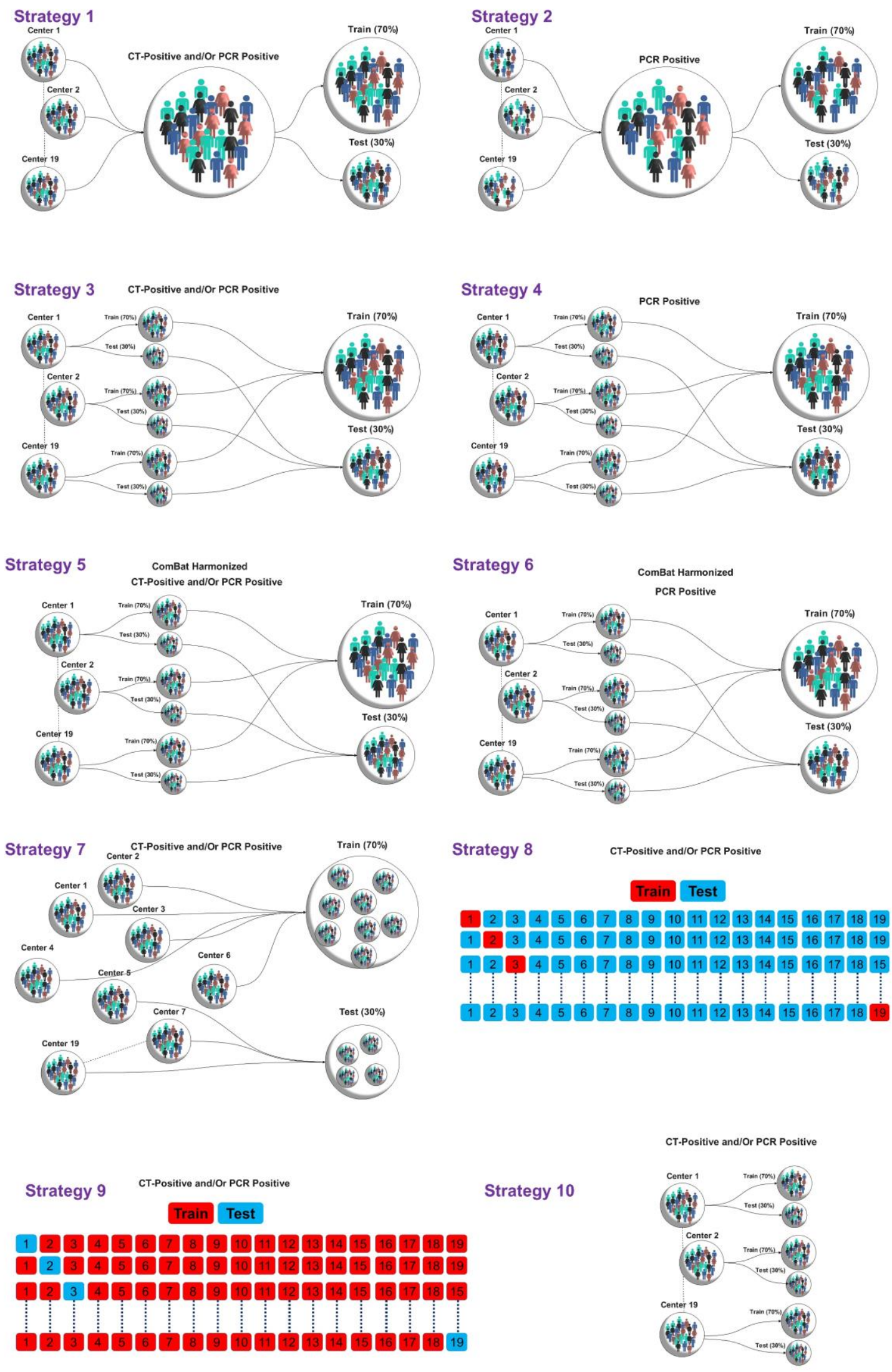

Figure 3: Different strategies implemented in this study for model evaluation 
medRxiv preprint doi: https://doi.org/10.1101/2021.12.07.21267364; this version posted December 7, 2021. The copyright holder for this preprint (which was not certified by peer review) is the author/funder, who has granted medRxiv a license to display the preprint in

It is made available under perpetuity.

perpetuity.

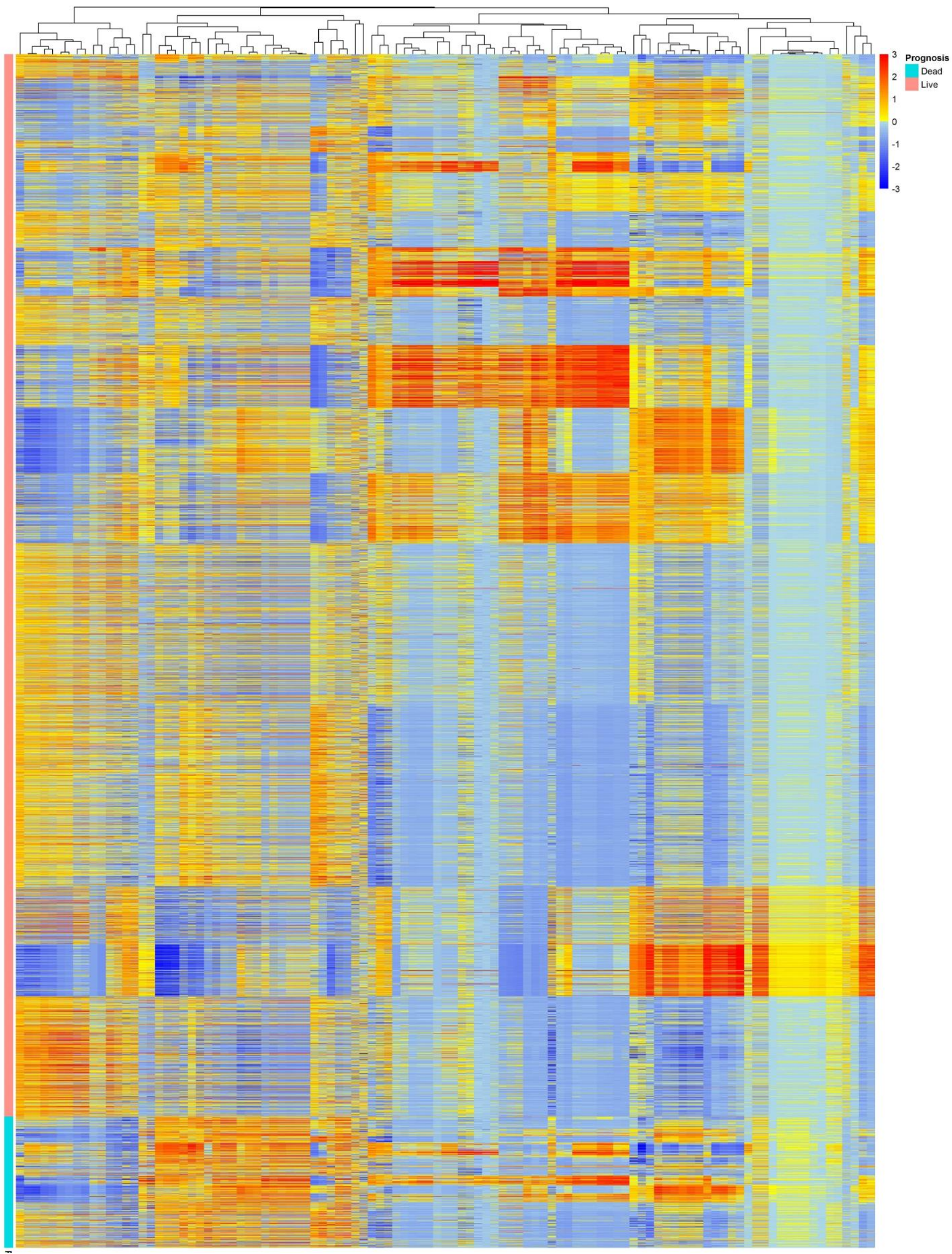

Figure 4: Cluster heat map of radiomics features in non-harmonized data set 
medRxiv preprint doi: https://doi.org/10.1101/2021.12.07.21267364; this version posted December 7, 2021. The copyright holder for this preprint (which was not certified by peer review) is the author/funder, who has granted medRxiv a license to display the preprint in

It is made available under a CC-BY-NC-ND 4.0 International license .

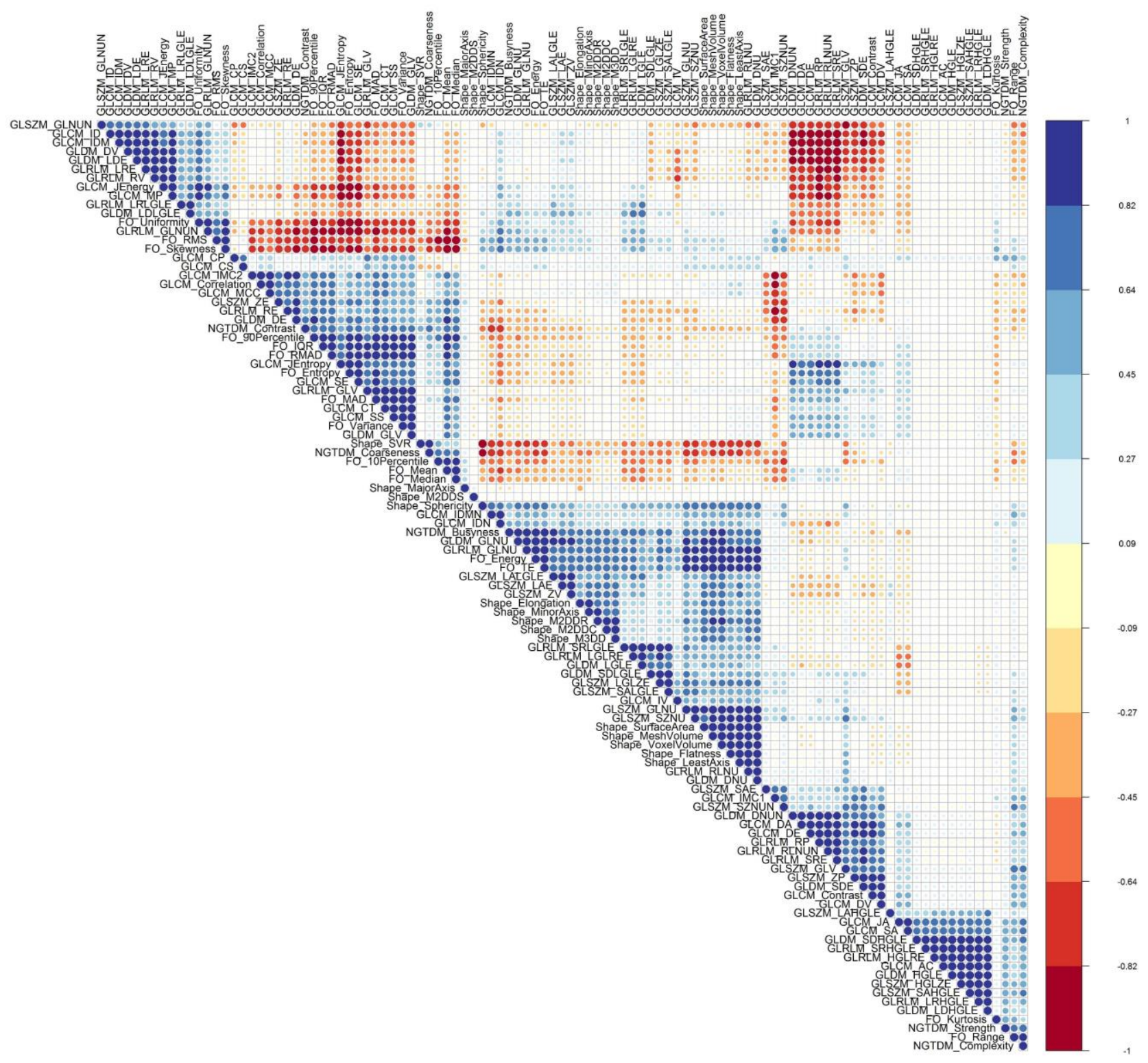

Figure 5. Radiomics feature correlation using Pearson correlation in non-harmonized data set 
AUC

\begin{tabular}{|c|c|c|c|c|c|c|c|c|c|c|}
\hline 0.81 & 0.83 & 0.81 & 0.82 & 0.79 & 0.78 & 0.75 & 0.69 & 0.79 & 0.80 & ANOVA_AB \\
\hline 0.83 & 0.84 & 0.83 & 0.84 & 0.82 & 0.82 & 0.78 & 0.72 & 0.80 & 0.79 & ANOVA_AE \\
\hline 0.82 & 0.83 & 0.83 & 0.83 & 0.77 & 0.78 & 0.76 & 0.74 & 0.80 & 0.81 & ANOVA_LASSO \\
\hline 0.82 & 0.81 & 0.81 & 0.82 & 0.77 & 0.77 & 0.76 & 0.71 & 0.79 & 0.82 & ANOVA_LDA \\
\hline 0.82 & 0.82 & 0.82 & 0.83 & 0.77 & 0.78 & 0.76 & 0.74 & 0.80 & 0.82 & ANOVA_LR \\
\hline 0.80 & 0.81 & 0.80 & 0.80 & 0.75 & 0.76 & 0.74 & 0.74 & 0.78 & 0.79 & ANOVA_NB \\
\hline 0.83 & 0.84 & 0.83 & 0.84 & 0.83 & 0.82 & 0.78 & 0.67 & 0.79 & 0.82 & ANOVA_RF \\
\hline 0.80 & 0.81 & 0.80 & 0.82 & 0.79 & 0.79 & 0.76 & 0.68 & 0.79 & 0.80 & KW_AB \\
\hline 0.83 & 0.82 & 0.80 & 0.84 & 0.81 & 0.82 & 0.77 & 0.72 & 0.81 & 0.79 & KW_AE \\
\hline 0.83 & 0.83 & 0.83 & 0.83 & 0.77 & 0.78 & 0.77 & 0.74 & 0.80 & 0.81 & KW LASSO \\
\hline 0.82 & 0.82 & 0.82 & 0.82 & 0.77 & 0.77 & 0.76 & 0.70 & 0.80 & 0.81 & KW LDA \\
\hline 0.82 & 0.83 & 0.81 & 0.82 & 0.77 & 0.78 & 0.76 & 0.72 & 0.80 & 0.81 & KW_LR \\
\hline 0.80 & 0.81 & 0.79 & 0.80 & 0.74 & 0.76 & 0.74 & 0.74 & 0.78 & 0.79 & KW_NB \\
\hline 0.83 & 0.84 & 0.81 & 0.84 & 0.82 & 0.82 & 0.78 & 0.67 & 0.79 & 0.82 & KW_RF \\
\hline 0.81 & 0.83 & 0.81 & 0.81 & 0.77 & 0.78 & 0.74 & 0.67 & 0.79 & 0.77 & Relief AB \\
\hline 0.83 & 0.84 & 0.83 & 0.83 & 0.79 & 0.81 & 0.76 & 0.71 & 0.80 & 0.79 & Relief $\mathrm{AE}$ \\
\hline 0.83 & 0.83 & 0.82 & 0.83 & 0.77 & 0.78 & 0.77 & 0.70 & 0.80 & 0.80 & Relief_LASSO \\
\hline 0.82 & 0.82 & 0.81 & 0.82 & 0.76 & 0.77 & 0.75 & 0.69 & 0.80 & 0.81 & Relief_LDA \\
\hline 0.82 & 0.82 & 0.81 & 0.82 & 0.77 & 0.78 & 0.76 & 0.71 & 0.79 & 0.81 & Relief_LR \\
\hline 0.79 & 0.80 & 0.79 & 0.79 & 0.75 & 0.75 & 0.73 & 0.64 & 0.75 & 0.79 & Relief_NB \\
\hline 0.84 & 0.84 & 0.83 & 0.83 & 0.83 & 0.83 & 0.78 & 0.66 & 0.79 & 0.83 & Relief RF \\
\hline 0.81 & 0.83 & 0.81 & 0.83 & 0.78 & 0.79 & 0.75 & 0.68 & 0.79 & 0.80 & RFE_AB \\
\hline 0.83 & 0.83 & 0.83 & 0.83 & 0.82 & 0.82 & 0.77 & 0.72 & 0.81 & 0.80 & RFE_AE \\
\hline 0.83 & 0.83 & 0.82 & 0.83 & 0.77 & 0.78 & 0.77 & 0.70 & 0.80 & 0.79 & RFE_LASSO \\
\hline 0.82 & 0.82 & 0.81 & 0.82 & 0.77 & 0.76 & 0.76 & 0.68 & 0.79 & 0.81 & RFE_LDA \\
\hline 0.82 & 0.83 & 0.82 & 0.82 & 0.77 & 0.78 & 0.76 & 0.70 & 0.80 & 0.80 & RFE_LR \\
\hline 0.79 & 0.80 & 0.79 & 0.79 & 0.75 & 0.76 & 0.74 & 0.67 & 0.76 & 0.79 & RFE_NB \\
\hline 0.84 & 0.84 & 0.83 & 0.84 & 0.83 & 0.83 & 0.79 & 0.67 & 0.79 & 0.82 & RFE RF \\
\hline $\begin{array}{l}\overline{0}_{1} \\
\text { के } \\
\stackrel{\Phi}{\pi} \\
\text { के }\end{array}$ & 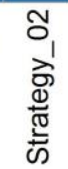 & 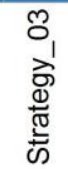 & 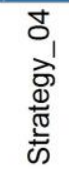 & 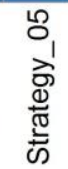 & 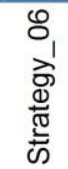 & 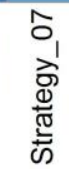 & 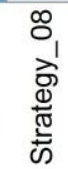 & 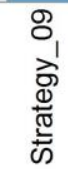 & 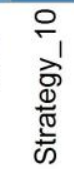 & \\
\hline
\end{tabular}

Figure 6: Heat map of AUC for cross combination of feature selectors and classifiers in different ten strategies 
Sensitivity

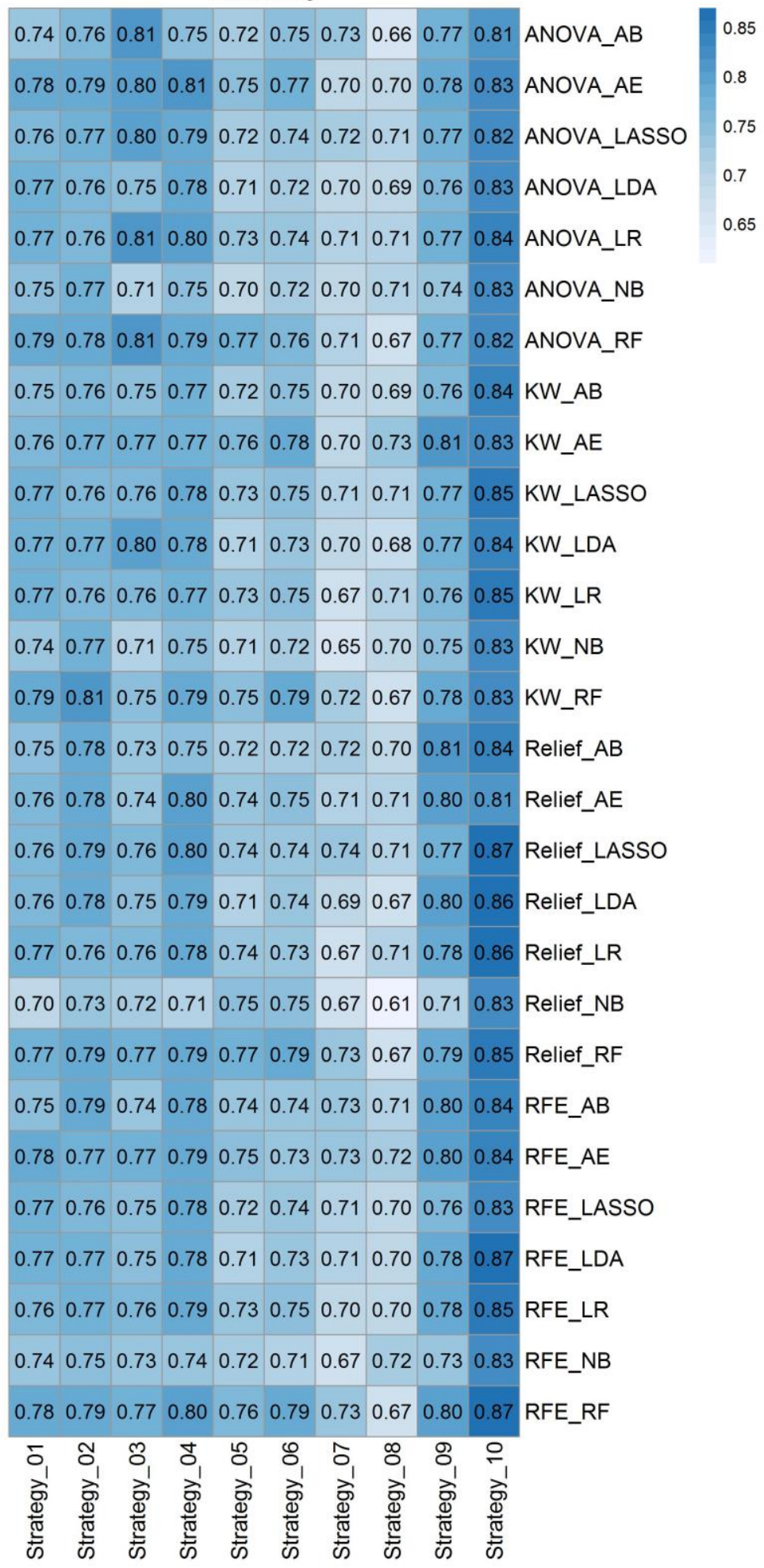

Figure 7: Heat map of Sensitivity for cross combination of feature selectors and classifiers in different ten strategies 


\section{Specificity}

\begin{tabular}{|c|c|c|c|c|c|c|c|c|c|c|}
\hline 0.73 & 0.76 & 0.68 & 0.75 & 0.75 & 0.73 & 0.66 & 0.64 & 0.74 & 0.76 & ANOVA_AB \\
\hline 0.74 & 0.75 & 0.72 & 0.72 & 0.77 & 0.73 & 0.73 & 0.65 & 0.76 & 0.73 & ANOVA_AE \\
\hline 0.76 & 0.75 & 0.72 & 0.75 & 0.73 & 0.74 & 0.71 & 0.68 & 0.75 & 0.78 & ANOVA_LASSO \\
\hline 0.73 & 0.74 & 0.75 & 0.74 & 0.72 & 0.72 & 0.72 & 0.66 & 0.75 & 0.77 & ANOVA_LDA \\
\hline 0.75 & 0.75 & 0.70 & 0.72 & 0.72 & 0.72 & 0.71 & 0.67 & 0.76 & 0.77 & ANOVA_LR \\
\hline 0.73 & 0.73 & 0.78 & 0.74 & 0.72 & 0.71 & 0.70 & 0.69 & 0.76 & 0.75 & ANOVA_NB \\
\hline 0.74 & 0.76 & 0.72 & 0.74 & 0.73 & 0.76 & 0.73 & 0.60 & 0.73 & 0.77 & ANOVA_RF \\
\hline 0.75 & 0.73 & 0.72 & 0.75 & 0.73 & 0.71 & 0.71 & 0.60 & 0.74 & 0.72 & KW_AB \\
\hline 0.76 & 0.74 & 0.70 & 0.77 & 0.75 & 0.73 & 0.74 & 0.62 & 0.73 & 0.72 & KW_AE \\
\hline 0.75 & 0.76 & 0.74 & 0.75 & 0.72 & 0.71 & 0.70 & 0.67 & 0.74 & 0.75 & KW_LASSO \\
\hline 0.74 & 0.75 & 0.72 & 0.74 & 0.72 & 0.71 & 0.71 & 0.64 & 0.74 & 0.74 & KW_LDA \\
\hline 0.74 & 0.76 & 0.74 & 0.74 & 0.71 & 0.71 & 0.76 & 0.65 & 0.76 & 0.75 & KW_LR \\
\hline 0.73 & 0.73 & 0.77 & 0.73 & 0.68 & 0.70 & 0.75 & 0.69 & 0.76 & 0.74 & KW_NB \\
\hline 0.74 & 0.76 & 0.72 & 0.74 & 0.75 & 0.73 & 0.72 & 0.59 & 0.71 & 0.75 & KW_RF \\
\hline 0.73 & 0.74 & 0.75 & 0.75 & 0.71 & 0.72 & 0.67 & 0.57 & 0.69 & 0.69 & Relief_AB \\
\hline 0.76 & 0.75 & 0.79 & 0.74 & 0.71 & 0.73 & 0.71 & 0.61 & 0.72 & 0.72 & Relief_AE \\
\hline 0.76 & 0.75 & 0.75 & 0.74 & 0.71 & 0.71 & 0.68 & 0.61 & 0.74 & 0.71 & Relief_LASSO \\
\hline 0.75 & 0.74 & 0.77 & 0.74 & 0.71 & 0.72 & 0.70 & 0.63 & 0.72 & 0.72 & Relief_LDA \\
\hline 0.73 & 0.74 & 0.73 & 0.73 & 0.71 & 0.72 & 0.74 & 0.62 & 0.74 & 0.71 & Relief_LR \\
\hline 0.77 & 0.75 & 0.74 & 0.76 & 0.65 & 0.66 & 0.72 & 0.63 & 0.74 & 0.72 & Relief_NB \\
\hline 0.76 & 0.76 & 0.76 & 0.74 & 0.74 & 0.72 & 0.72 & 0.58 & 0.71 & 0.74 & Relief_RF \\
\hline 0.73 & 0.74 & 0.75 & 0.74 & 0.70 & 0.73 & 0.67 & 0.58 & 0.70 & 0.74 & RFE_AB \\
\hline 0.74 & 0.76 & 0.76 & 0.76 & 0.75 & 0.80 & 0.70 & 0.62 & 0.73 & 0.72 & RFE_AE \\
\hline 0.75 & 0.75 & 0.77 & 0.74 & 0.73 & 0.71 & 0.71 & 0.61 & 0.76 & 0.72 & RFE_LASSO \\
\hline 0.74 & 0.74 & 0.75 & 0.74 & 0.72 & 0.72 & 0.70 & 0.60 & 0.73 & 0.71 & RFE_LDA \\
\hline 0.74 & 0.75 & 0.74 & 0.72 & 0.71 & 0.71 & 0.73 & 0.62 & 0.75 & 0.71 & RFE_LR \\
\hline 0.71 & 0.74 & 0.72 & 0.73 & 0.69 & 0.71 & 0.73 & 0.57 & 0.74 & 0.74 & RFE_NB \\
\hline 0.76 & 0.75 & 0.74 & 0.73 & 0.74 & 0.74 & 0.71 & 0.60 & 0.70 & 0.70 & RFE_RF \\
\hline 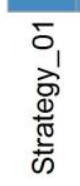 & 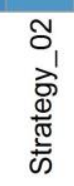 & 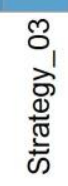 & 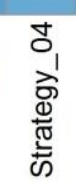 & 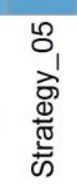 & 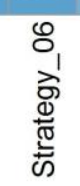 & 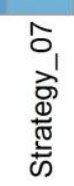 & 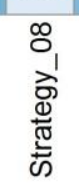 & 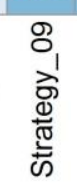 & 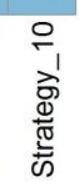 & \\
\hline
\end{tabular}

Figure 8: Heat map of Specificity for cross combination of feature selectors and classifiers in different ten strategies 
a.

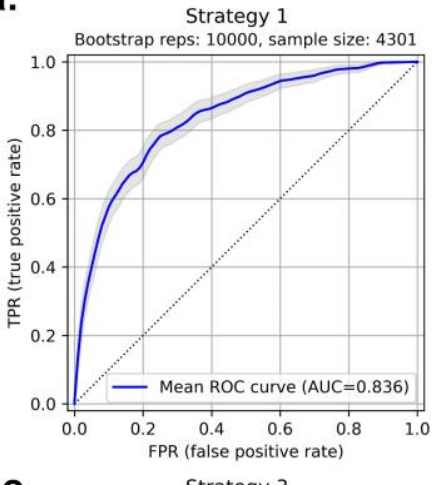

C.

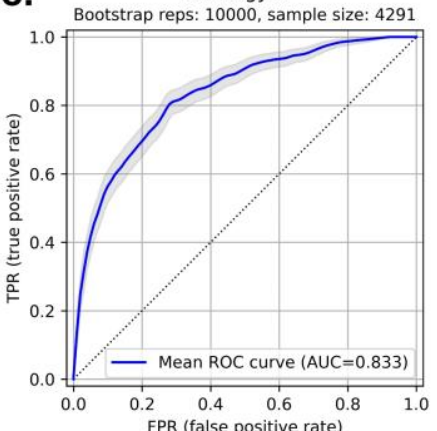

e.

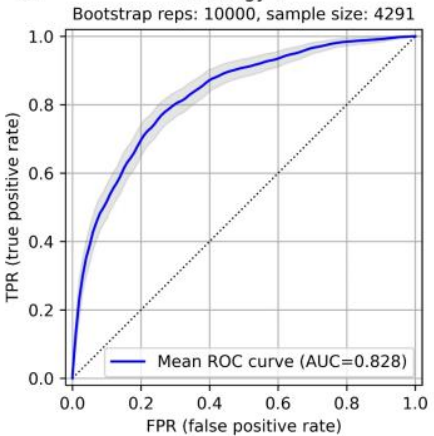

g.

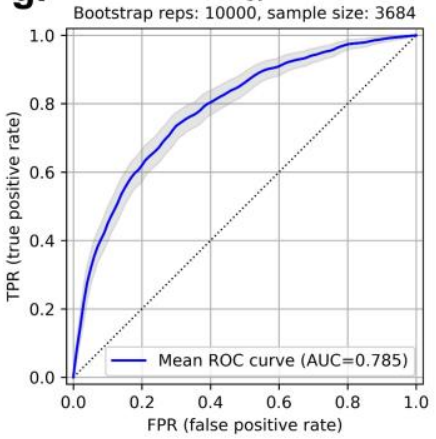

b.

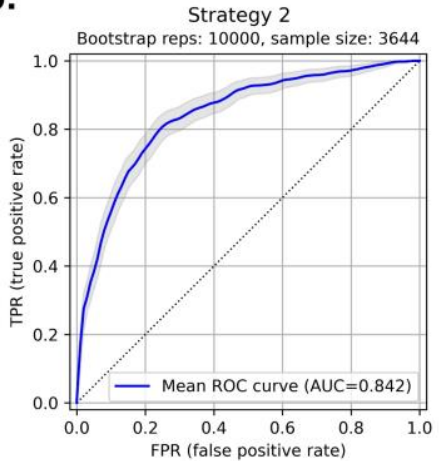

d.

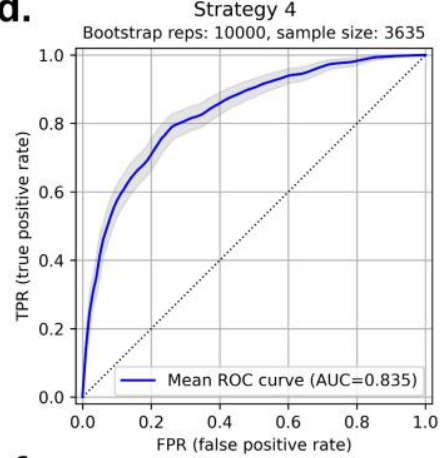

f.

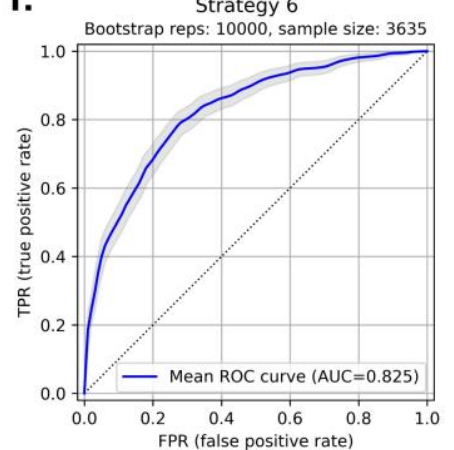

k.

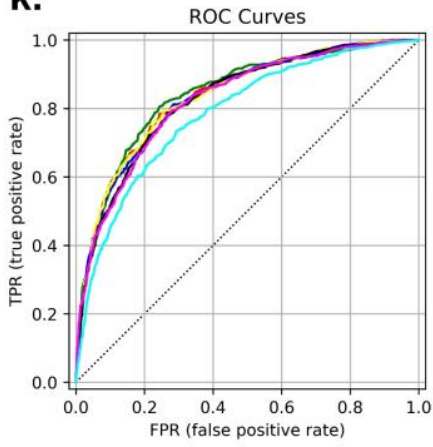

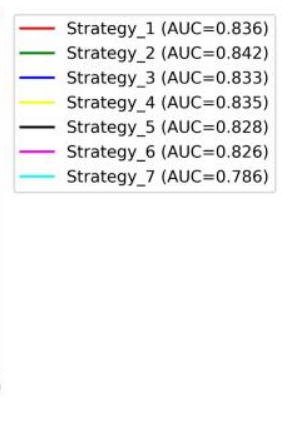

Figure 9: ROC curve for test sets in strategies 1-7. Strategy 1: AUC 0.84 \pm 0.01 (a), Strategy 2: AUC 0.84 \pm 0.01 (b), Strategy 3: AUC 0.83 \pm 0.01 (c), Strategy 4: AUC 0.83 \pm 0.01 (d), Strategy 5: AUC 0.83 \pm 0.01 (e), Strategy 6: AUC 0.83 \pm 0.01 (f), Strategy 7: AUC 0.79 \pm 0.01 (g) and different strategies comparison $(\mathrm{k})$ 
TRIPOD Checklist: Prediction Model Development and Validation.

Title and abstract

\begin{tabular}{|c|l|l|c|c|}
\hline Title & 1 & $\mathrm{D} ; \mathrm{V}$ & $\begin{array}{c}\text { Identify the study as developing and/or validating a multivariable prediction model, the target } \\
\text { population, and the outcome to be predicted. }\end{array}$ & $\begin{array}{r}\text { Provide a summary of objectives, study design, setting, participants, sample size, predictors, outcome, } \\
\text { statistical analysis, results, and conclusions. }\end{array}$ \\
\hline Abstract & 2 & $\mathrm{D} ; \mathrm{V}$ & Introduction & \begin{tabular}{c}
1 \\
\hline
\end{tabular}
\end{tabular}

\begin{tabular}{|c|c|c|c|c|}
\hline \multirow{2}{*}{$\begin{array}{l}\text { Background and } \\
\text { objectives }\end{array}$} & $3 \mathrm{a}$ & $\mathrm{D} ; \mathrm{V}$ & $\begin{array}{l}\text { Explain the medical context (including whether diagnostic or prognostic) and rationale for developing } \\
\text { or validating the multivariable prediction model, including references to existing models. }\end{array}$ & $2-4$ \\
\hline & $3 b$ & $\mathrm{D} ; \mathrm{V}$ & $\begin{array}{l}\text { Specify the objectives, including whether the study describes the development or validation of the } \\
\text { model or both. }\end{array}$ & 4 \\
\hline
\end{tabular}

\begin{tabular}{|c|c|c|c|c|}
\hline \multirow{2}{*}{\multicolumn{5}{|c|}{ model or both. }} \\
\hline & & & & \\
\hline \multirow[t]{2}{*}{ Source of data } & $4 a$ & $\mathrm{D} ; \mathrm{V}$ & $\begin{array}{l}\text { Describe the study design or source of data (e.g., randomized trial, cohort, or registry data), separately } \\
\text { for the development and validation data sets, if applicable. }\end{array}$ & 5 \\
\hline & $4 \mathrm{~b}$ & $\mathrm{D} ; \mathrm{V}$ & Specify the key study dates, including start of accrual; end of accrual; and, if applicable, end of follow- & 6 \\
\hline
\end{tabular}

\begin{tabular}{|c|c|c|c|c|}
\hline & & & up. & \\
\hline \multirow{3}{*}{ Participants } & $5 \mathrm{a}$ & $\mathrm{D} ; \mathrm{V}$ & $\begin{array}{l}\text { Specify key elements of the study setting (e.g., primary care, secondary care, general population) } \\
\text { including number and location of centres. }\end{array}$ & 6 \\
\hline & $5 \mathrm{~b}$ & $\mathrm{D} ; \mathrm{V}$ & Describe eligibility criteria for participants. & 5 \\
\hline & $5 \mathrm{c}$ & $\mathrm{D} ; \mathrm{V}$ & Give details of treatments received, if relevant. & 6 \\
\hline \multirow{2}{*}{ Outcome } & $6 \mathrm{a}$ & $\mathrm{D} ; \mathrm{V}$ & Clearly define the outcome that is predicted by the prediction model, including how and when assessed. & 6 \\
\hline & $6 \mathrm{~b}$ & $\mathrm{D} ; \mathrm{V}$ & Report any actions to blind assessment of the outcome to be predicted. & $8-10$ \\
\hline \multirow[t]{2}{*}{ Predictors } & $7 \mathrm{a}$ & $\mathrm{D} ; \mathrm{V}$ & $\begin{array}{l}\text { Clearly define all predictors used in developing or validating the multivariable prediction model, } \\
\text { including how and when they were measured. }\end{array}$ & 7 \\
\hline & $7 b$ & $\mathrm{D} ; \mathrm{V}$ & Report any actions to blind assessment of predictors for the outcome and other predictors. & $8-10$ \\
\hline Sample size & 8 & $\mathrm{D} ; \mathrm{V}$ & Explain how the study size was arrived at. & $5-6$ \\
\hline Missing data & 9 & $\mathrm{D} ; \mathrm{V}$ & $\begin{array}{l}\text { Describe how missing data were handled (e.g., complete-case analysis, single imputation, multiple } \\
\text { imputation) with details of any imputation method. }\end{array}$ & NA \\
\hline \multirow{5}{*}{$\begin{array}{l}\text { Statistical analysis } \\
\text { methods }\end{array}$} & $10 \mathrm{a}$ & $\mathrm{D}$ & Describe how predictors were handled in the analyses. & 7 \\
\hline & $10 \mathrm{~b}$ & $\mathrm{D}$ & $\begin{array}{l}\text { Specify type of model, all model-building procedures (including any predictor selection), and method } \\
\text { for internal validation. }\end{array}$ & $8-10$ \\
\hline & $10 \mathrm{c}$ & $\mathrm{V}$ & For validation, describe how the predictions were calculated. & $8-10$ \\
\hline & $10 \mathrm{~d}$ & $\mathrm{D} ; \mathrm{V}$ & Specify all measures used to assess model performance and, if relevant, to compare multiple models. & $8-10$ \\
\hline & $10 \mathrm{e}$ & $\mathrm{V}$ & Describe any model updating (e.g., recalibration) arising from the validation, if done. & $8-9$ \\
\hline Risk groups & 11 & $\mathrm{D} ; \mathrm{V}$ & Provide details on how risk groups were created, if done. & $6-7$ \\
\hline $\begin{array}{l}\text { Development vs. } \\
\text { validation }\end{array}$ & 12 & V & $\begin{array}{l}\text { For validation, identify any differences from the development data in setting, eligibility criteria, } \\
\text { outcome, and predictors. }\end{array}$ & $\begin{array}{c}\text { 8-9 } \\
\text { Fig. } 3\end{array}$ \\
\hline
\end{tabular}

\begin{tabular}{|c|c|c|c|c|}
\hline \multicolumn{5}{|c|}{ Results } \\
\hline \multirow{3}{*}{ Participants } & $13 \mathrm{a}$ & $\mathrm{D} ; \mathrm{V}$ & $\begin{array}{l}\text { Describe the flow of participants through the study, including the number of participants with and } \\
\text { without the outcome and, if applicable, a summary of the follow-up time. A diagram may be helpful. }\end{array}$ & 5-6 \\
\hline & $13 b$ & $\mathrm{D} ; \mathrm{V}$ & $\begin{array}{l}\text { Describe the characteristics of the participants (basic demographics, clinical features, available } \\
\text { predictors), including the number of participants with missing data for predictors and outcome. Not } \\
\text { mentioned in result }\end{array}$ & $\begin{array}{c}5-6 \\
\text { Table } 1\end{array}$ \\
\hline & $13 \mathrm{c}$ & $\mathrm{V}$ & $\begin{array}{l}\text { For validation, show a comparison with the development data of the distribution of important variables } \\
\text { (demographics, predictors and outcome). }\end{array}$ & $10-12$ \\
\hline \multirow[t]{2}{*}{ Model development } & $14 \mathrm{a}$ & $\mathrm{D}$ & Specify the number of participants and outcome events in each analysis. & $\begin{array}{c}5-6 \\
\text { Fig. } 2\end{array}$ \\
\hline & $14 \mathrm{~b}$ & $\mathrm{D}$ & If done, report the unadjusted association between each candidate predictor and outcome. & 10 \\
\hline
\end{tabular}




\begin{tabular}{|c|c|c|c|c|}
\hline \multirow[t]{2}{*}{ Model specification } & $15 \mathrm{a}$ & $\mathrm{D}$ & $\begin{array}{l}\text { Present the full prediction model to allow predictions for individuals (i.e., all regression coefficients, } \\
\text { and model intercept or baseline survival at a given time point). }\end{array}$ & NA \\
\hline & $15 b$ & $\mathrm{D}$ & Explain how to the use the prediction model. & $10-12$ \\
\hline Model performance & 16 & $\mathrm{D} ; \mathrm{V}$ & Report performance measures (with CIs) for the prediction model. & $10-12$ \\
\hline Model-updating & 17 & $\mathrm{~V}$ & If done, report the results from any model updating (i.e., model specification, model performance). & $10-11$ \\
\hline \multicolumn{5}{|c|}{ Discussion } \\
\hline Limitations & 18 & $\mathrm{D} ; \mathrm{V}$ & $\begin{array}{l}\text { Discuss any limitations of the study (such as nonrepresentative sample, few events per predictor, } \\
\text { missing data). }\end{array}$ & 15 \\
\hline \multirow[t]{2}{*}{ Interpretation } & $19 \mathrm{a}$ & $\mathrm{V}$ & $\begin{array}{c}\text { For validation, discuss the results with reference to performance in the development data, and any other } \\
\text { validation data. }\end{array}$ & $12-14$ \\
\hline & $19 \mathrm{~b}$ & $\mathrm{D} ; \mathrm{V}$ & $\begin{array}{l}\text { Give an overall interpretation of the results, considering objectives, limitations, results from similar } \\
\text { studies, and other relevant evidence. }\end{array}$ & $14-15$ \\
\hline Implications & 20 & $\mathrm{D} ; \mathrm{V}$ & Discuss the potential clinical use of the model and implications for future research. & $15-16$ \\
\hline \multicolumn{5}{|c|}{ Other information } \\
\hline $\begin{array}{l}\text { Supplementary } \\
\text { information }\end{array}$ & 21 & $\mathrm{D} ; \mathrm{V}$ & $\begin{array}{l}\text { Provide information about the availability of supplementary resources, such as study protocol, Web } \\
\text { calculator, and data sets. }\end{array}$ & 16 \\
\hline Funding & 22 & $\mathrm{D} ; \mathrm{V}$ & Give the source of funding and the role of the funders for the present study. & 16 \\
\hline
\end{tabular}

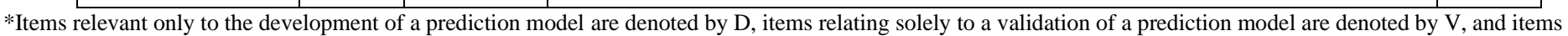
relating to both are denoted $\mathrm{D} ; \mathrm{V}$. We recommend using the TRIPOD Checklist in conjunction with the TRIPOD Explanation and Elaboration document. 


\section{Radiomics Quality Score - RQS}

$8 / 23 / 2021$

RQS - Radiomics.world

Image protocol quality - well-documented image protocols (for example, contrast, slice thickness, energy, etc.) and/or usage of public image protocols allow reproducibility/replicability

$\checkmark$ protocols well documented

$\checkmark$ public protocol used

$\square$ none

Multiple segmentations - possible actions are: segmentation by different physicians/algorithms/software, perturbing segmentations by (random) noise, segmentation at different breathing cycles. Analyse feature robustness to segmentation variabilities

$\bigcirc$ yes

() no

Phantom study on all scanners - detect inter-scanner differences and vendor-dependent features. Analyse feature robustness to these sources of variability

$\bigcirc$ yes

(1) no

Imaging at multiple time points - collect images of individuals at additional time points. Analyse feature robustness to temporal variabilities (for example, organ movement, organ expansion/shrinkage)

yes

(1) no

Feature reduction or adjustment for multiple testing - decreases the risk of overfitting. Overfitting is inevitable if the number of features exceeds the number of samples. Consider feature robustness when selecting features

(]) Either measure is implemented

Neither measure is implemented

Multivariable analysis with non radiomics features (for example, EGFR mutation) - is expected to provide a more holistic model. Permits correlating/inferencing between radiomics and non radiomics features

yes

$\bigcirc$ no

Detect and discuss biological correlates - demonstration of phenotypic differences (possibly associated with underlying gene-protein expression patterns) deepens understanding of radiomics and biology

(ㄱes

no

Cut-off analyses - determine risk groups by either the median, a previously published cut-off or report a continuous risk variable. Reduces the risk of reporting overly optimistic results

$\bigcirc$ yes

(1) no

Discrimination statistics - report discrimination statistics (for example, C-statistic, ROC curve, AUC) and their statistical significance (for example, p-values, confidence intervals). One can also apply resampling method (for example, bootstrapping, cross-validation)

$\checkmark$ a discrimination statistic and its statistical significance are reported

$\checkmark$ a resampling method technique is also applied

none

https://www.radiomics.world/rqs 
Calibration statistics - report calibration statistics (for example, Calibration-in-the-large/slope, calibration plots) and their statistical significance (for example, P-values, confidence intervals). One can also apply resampling method (for example, bootstrapping, cross-validation)

$\nabla$ a calibration statistic and its statistical significance are reported

$\checkmark$ a resampling method technique is applied

$\square$ none

Prospective study registered in a trial database - provides the highest level of evidence supporting the clinical validity and usefulness of the radiomics biomarker

yes

$\bigcirc$ no

Validation - the validation is performed without retraining and without adaptation of the cut-off value, provides crucial information with regard to credible clinical performance

$\square$ No validation

validation is based on a dataset from the same institute

validation is based on a dataset from another institute

validation is based on two datasets from two distinct institutes

the study validates a previously published signature

$\checkmark$ validation is based on three or more datasets from distinct institutes

Comparison to 'gold standard' - assess the extent to which the model agrees with/is superior to the current 'gold standard' method (for example, TNM-staging for survival prediction). This comparison shows the added value of radiomics

(O) yes

no

Potential clinical utility - report on the current and potential application of the model in a clinical setting (for example, decision curve analysis).

O yes

$\bigcirc$ no

Cost-effectiveness analysis - report on the cost-effectiveness of the clinical application (for example, QALYs generated)

(O) yes

$\bigcirc$ no

Open science and data - make code and data publicly available. Open science facilitates knowledge transfer and reproducibility of the study

$\square$ scans are open source

region of interest segmentations are open source

$\checkmark$ the code is open sourced

$\checkmark$ radiomics features are calculated on a set of representative ROIs and the calculated features and representative ROIs are open source

https://www.radiomics.world/rqs

RQS - Radiomics.world

Total score

30

$(83.33 \%)$ 


\section{CLAIM: Checklist for Artificial Intelligence in Medical Imaging}

\begin{tabular}{|c|c|c|c|}
\hline Section / Topic & No. & Item & \\
\hline \multicolumn{4}{|l|}{ TITLE / ABSTRACT } \\
\hline & 1 & $\begin{array}{l}\text { Identification as a study of AI methodology, specifying the category of technology used (e.g., deep } \\
\text { learning) }\end{array}$ & $\checkmark$ \\
\hline & 2 & Structured summary of study design, methods, results, and conclusions & $\checkmark$ \\
\hline \multicolumn{4}{|l|}{ INTRODUCTION } \\
\hline & 3 & Scientific and clinical background, including the intended use and clinical role of the AI approach & $\checkmark$ \\
\hline & 4 & Study objectives and hypotheses & $\checkmark$ \\
\hline \multicolumn{4}{|l|}{ METHODS } \\
\hline \multirow[t]{2}{*}{ Study Design } & 5 & Prospective or retrospective study & $\checkmark$ \\
\hline & 6 & Study goal, such as model creation, exploratory study, feasibility study, non-inferiority trial & $\checkmark$ \\
\hline \multirow[t]{7}{*}{ Data } & 7 & Data sources & $\checkmark$ \\
\hline & 8 & $\begin{array}{l}\text { Eligibility criteria: how, where, and when potentially eligible participants or studies were identified } \\
\text { (e.g., symptoms, results from previous tests, inclusion in registry, patient-care setting, location, } \\
\text { dates) }\end{array}$ & $\checkmark$ \\
\hline & 9 & Data pre-processing steps & $\checkmark$ \\
\hline & 10 & Selection of data subsets, if applicable & $\checkmark$ \\
\hline & 11 & Definitions of data elements, with references to Common Data Elements & $\checkmark$ \\
\hline & 12 & De-identification methods & $\checkmark$ \\
\hline & 13 & How missing data were handled & $\checkmark$ \\
\hline \multirow[t]{5}{*}{ Ground Truth } & 14 & Definition of ground truth reference standard, in sufficient detail to allow replication & $\checkmark$ \\
\hline & 15 & Rationale for choosing the reference standard (if alternatives exist) & $\checkmark$ \\
\hline & 16 & Source of ground-truth annotations; qualifications and preparation of annotators & $\checkmark$ \\
\hline & 17 & Annotation tools & $\checkmark$ \\
\hline & 18 & $\begin{array}{l}\text { Measurement of inter- and intrarater variability; methods to mitigate variability and/or resolve } \\
\text { discrepancies }\end{array}$ & NA \\
\hline \multirow[t]{3}{*}{ Data Partitions } & 19 & Intended sample size and how it was determined & $\checkmark$ \\
\hline & 20 & How data were assigned to partitions; specify proportions & $\checkmark$ \\
\hline & 21 & Level at which partitions are disjoint (e.g., image, study, patient, institution) & $\checkmark$ \\
\hline \multirow[t]{3}{*}{ Model } & 22 & Detailed description of model, including inputs, outputs, all intermediate layers and connections & $\checkmark$ \\
\hline & 23 & Software libraries, frameworks, and packages & $\checkmark$ \\
\hline & 24 & Initialization of model parameters (e.g., randomization, transfer learning) & $\checkmark$ \\
\hline \multirow[t]{3}{*}{ Training } & 25 & $\begin{array}{l}\text { Details of training approach, including data augmentation, hyperparameters, number of models } \\
\text { trained }\end{array}$ & $\checkmark$ \\
\hline & 26 & Method of selecting the final model & $\checkmark$ \\
\hline & 27 & Ensembling techniques, if applicable & $\checkmark$ \\
\hline \multirow[t]{5}{*}{ Evaluation } & 28 & Metrics of model performance & $\checkmark$ \\
\hline & 29 & Statistical measures of significance and uncertainty (e.g., confidence intervals) & $\checkmark$ \\
\hline & 30 & Robustness or sensitivity analysis & $\checkmark$ \\
\hline & 31 & Methods for explainability or interpretability (e.g., saliency maps), and how they were validated & NA \\
\hline & 32 & Validation or testing on external data & $\checkmark$ \\
\hline \multicolumn{4}{|l|}{ RESULTS } \\
\hline \multirow[t]{2}{*}{ Data } & 33 & Flow of participants or cases, using a diagram to indicate inclusion and exclusion & $\checkmark$ \\
\hline & 34 & Demographic and clinical characteristics of cases in each partition & $\checkmark$ \\
\hline \multirow[t]{3}{*}{ Model performance } & 35 & Performance metrics for optimal model(s) on all data partitions & $\checkmark$ \\
\hline & 36 & Estimates of diagnostic accuracy and their precision (such as $95 \%$ confidence intervals) & $\checkmark$ \\
\hline & 37 & Failure analysis of incorrectly classified cases & NA \\
\hline \multicolumn{4}{|l|}{ DISCUSSION } \\
\hline & 38 & Study limitations, including potential bias, statistical uncertainty, and generalizability & $\checkmark$ \\
\hline & 39 & Implications for practice, including the intended use and/or clinical role & $\checkmark$ \\
\hline \multicolumn{4}{|l|}{ OTHER INFORMATION } \\
\hline & 40 & Registration number and name of registry & $\checkmark$ \\
\hline & 41 & Where the full study protocol can be accessed & $\checkmark$ \\
\hline & 42 & Sources of funding and other support; role of funders & $\checkmark$ \\
\hline
\end{tabular}




\section{$\underline{\text { Supplemental Figures and Tables }}$}




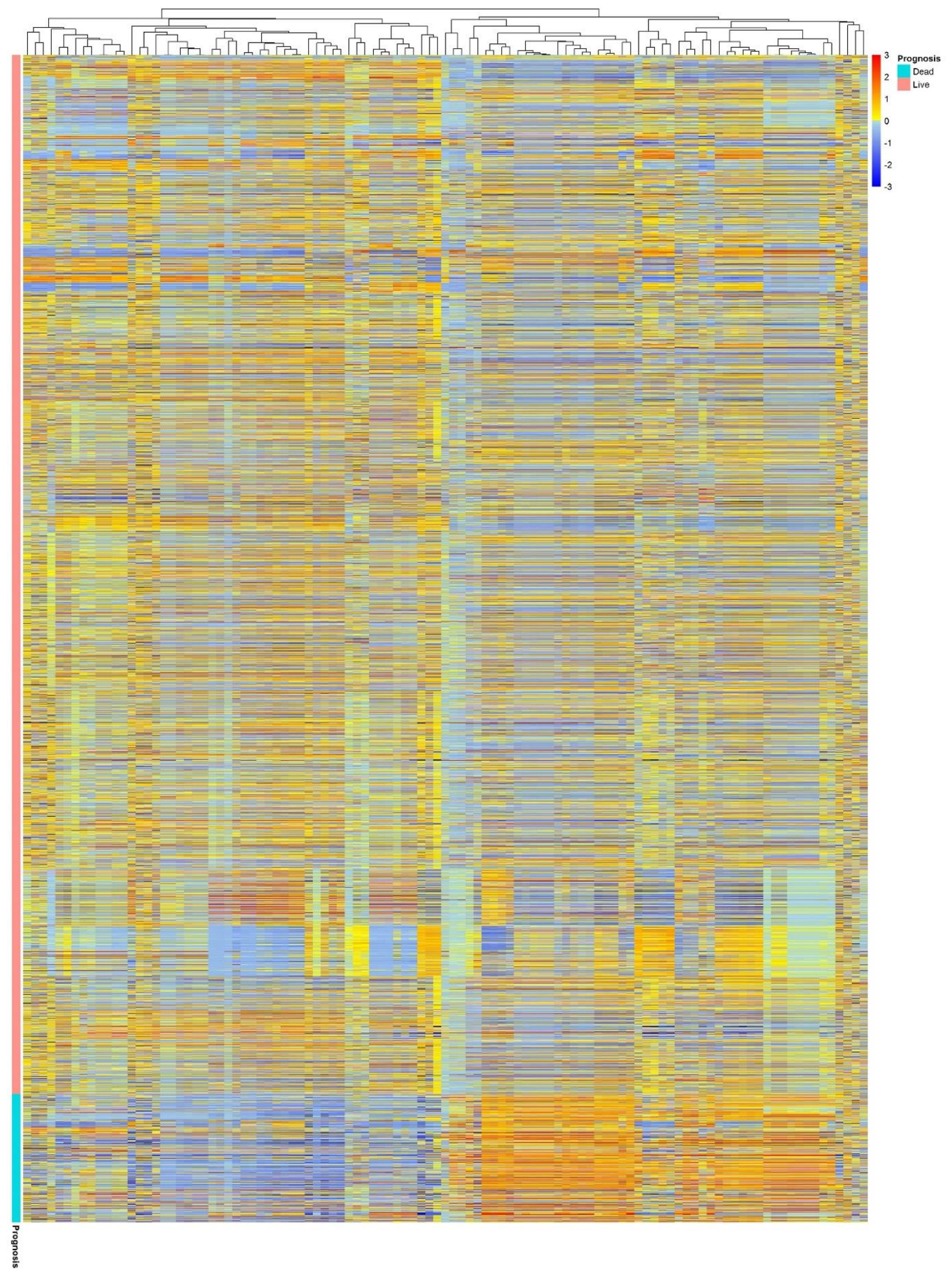

Supplemental Figure 1. Cluster heat map of radiomics features in the non-harmonized data set. 


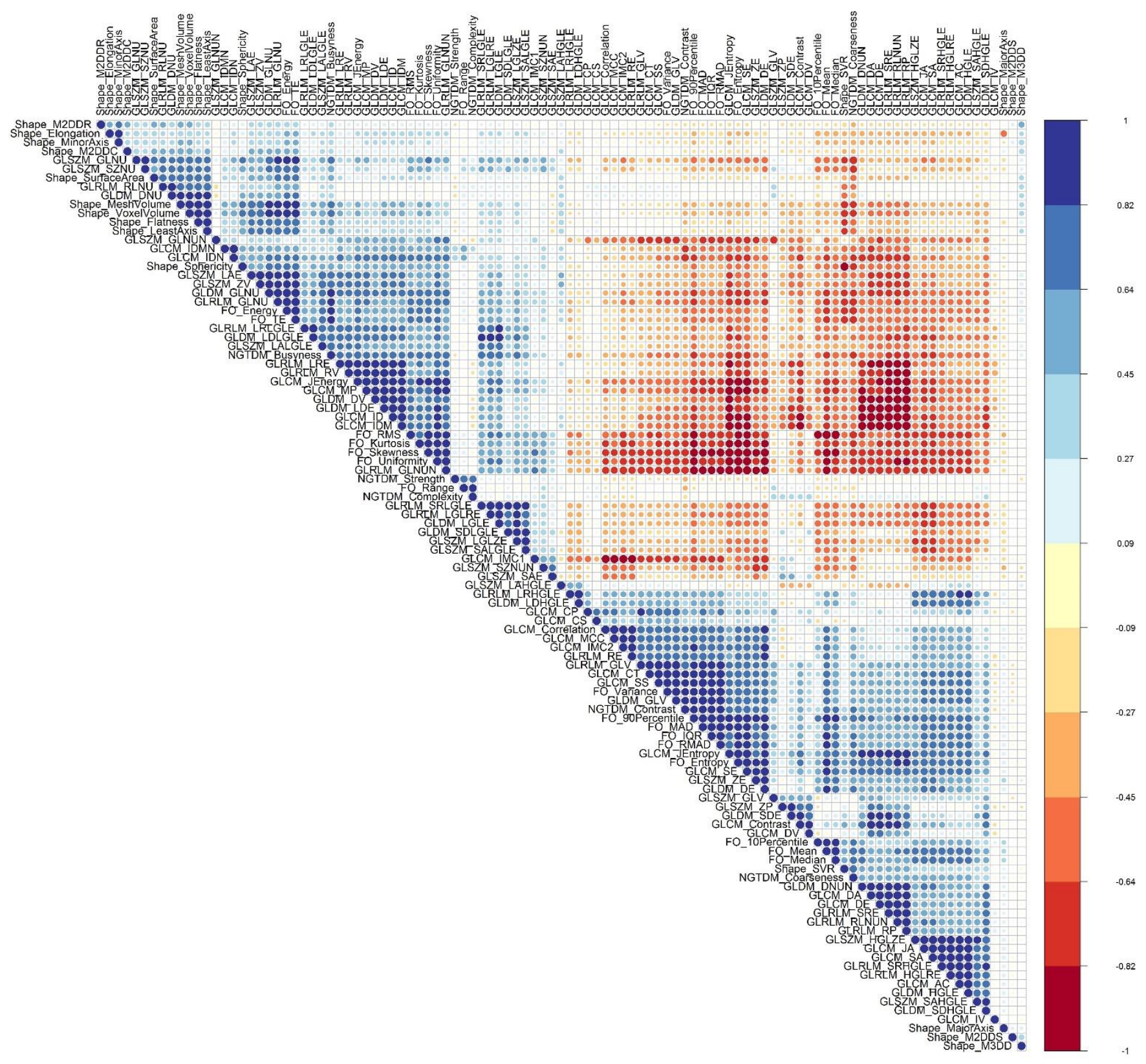

Supplemental Figure 2. Pearson correlation of ComBat-harmonized radiomics features. 
Supplemental Table 1. P-values in Kruskal Wallis analysis of radiomics features before and after ComBat Harmonization.

\begin{tabular}{|c|c|c|c|c|c|c|c|c|c|c|c|c|c|c|c|c|c|}
\hline Shape & Before & After & FO & Before & After & GLCM & Before & After & GLRLM & Before & After & GLSZM & Before & After & GLDM & Before & After \\
\hline Elongation & $1 \mathrm{E}-340$ & 0.01 & 10Percentile & $1 \mathrm{E}-340$ & 0.90 & AC & $1 \mathrm{E}-340$ & $3.14 \mathrm{E}-35$ & GLNU & 1E-340 & 0.76 & GLNU & $1 \mathrm{E}-340$ & 0.533587 & DE & $1 \mathrm{E}-340$ & 0.38 \\
\hline Flatness & $1 \mathrm{E}-340$ & $2.09 \mathrm{E}-28$ & 90Percentile & $1 \mathrm{E}-340$ & 0.78 & $\mathbf{C P}$ & $1 \mathrm{E}-340$ & $4.16 \mathrm{E}-62$ & GLNUN & $1 \mathrm{E}-340$ & 0.93 & GLNUN & $1 \mathrm{E}-340$ & 0.038013 & DNU & $1 \mathrm{E}-340$ & $2.18 \mathrm{E}-18$ \\
\hline LeastAxis & $1 \mathrm{E}-340$ & 2.13E-34 & Energy & $1 \mathrm{E}-340$ & 0.96 & CS & $1 \mathrm{E}-340$ & $4.98 \mathrm{E}-09$ & GLV & $1 \mathrm{E}-340$ & 0.97 & GLV & $1 \mathrm{E}-340$ & $6.14 \mathrm{E}-13$ & DNUN & $1 \mathrm{E}-340$ & 0.99 \\
\hline MajorAxis & $1 \mathrm{E}-340$ & 0.92 & Entropy & $1 \mathrm{E}-340$ & 0.99 & CT & $1 \mathrm{E}-340$ & 0.93 & HGLRE & $1 \mathrm{E}-340$ & $1.04 \mathrm{E}-21$ & HGLZE & $1 \mathrm{E}-340$ & $5.46 \mathrm{E}-07$ & DV & $1 \mathrm{E}-340$ & 0.06 \\
\hline M2DDC & $1 \mathrm{E}-340$ & $1.36 \mathrm{E}-110$ & IQR & $1 \mathrm{E}-340$ & 0.99 & Contrast & $1 \mathrm{E}-340$ & 0.95 & LRE & 1E-340 & 0.99 & LAE & $1 \mathrm{E}-340$ & $1.14 \mathrm{E}-19$ & GLNU & $1 \mathrm{E}-340$ & 0.01 \\
\hline M2DDR & $1 \mathrm{E}-340$ & $2.74 \mathrm{E}-93$ & Kurtosis & $1 \mathrm{E}-340$ & 7.95E-13 & Correlation & $1 \mathrm{E}-340$ & 0.89 & LRHGLE & $1 \mathrm{E}-340$ & $3.66 \mathrm{E}-112$ & LAHGLE & $1 \mathrm{E}-340$ & $2.10 \mathrm{E}-64$ & GLV & $1 \mathrm{E}-340$ & 0.98 \\
\hline M2DDS & $1 \mathrm{E}-340$ & 0.54 & MAD & $1 \mathrm{E}-340$ & 0.99 & DA & $1 \mathrm{E}-340$ & 0.99 & LRLGLE & $1 \mathrm{E}-340$ & $2.84 \mathrm{E}-42$ & LALGLE & $1 \mathrm{E}-340$ & $1.29 \mathrm{E}-156$ & HGLE & $1 \mathrm{E}-340$ & $1.07 \mathrm{E}-29$ \\
\hline M3DD & $1 \mathrm{E}-340$ & $1.54 \mathrm{E}-06$ & Mean & $1 \mathrm{E}-340$ & 0.99 & DE & $1 \mathrm{E}-340$ & 0.99 & LGLRE & $1 \mathrm{E}-340$ & 0.39 & LGLZE & $1 \mathrm{E}-340$ & 0.25 & LDE & $1 \mathrm{E}-340$ & 0.96 \\
\hline MeshVolume & $1 \mathrm{E}-340$ & $2.22 \mathrm{E}-05$ & Median & $1 \mathrm{E}-340$ & 0.99 & DV & $1 \mathrm{E}-340$ & 0.06 & RE & $1 \mathrm{E}-340$ & 0.90 & SZNU & $1 \mathrm{E}-340$ & 0.01 & LDHGLE & $1 \mathrm{E}-340$ & $1.76 \mathrm{E}-144$ \\
\hline MinorAxis & $1 \mathrm{E}-340$ & $8.82 \mathrm{E}-10$ & Range & $1 \mathrm{E}-340$ & $1.56 \mathrm{E}-06$ & ID & $1 \mathrm{E}-340$ & 0.99 & RLNU & $1 \mathrm{E}-340$ & $1.80 \mathrm{E}-12$ & SZNUN & 1E-340 & 0.63 & LDLGLE & $1 \mathrm{E}-340$ & $1.68 \mathrm{E}-05$ \\
\hline Sphericity & $1 \mathrm{E}-340$ & $3.92 \mathrm{E}-07$ & RMAD & $1 \mathrm{E}-340$ & 0.99 & IDM & $1 \mathrm{E}-340$ & 0.99 & RLNUN & $1 \mathrm{E}-340$ & 0.99 & SAE & $1 \mathrm{E}-340$ & $2.56 \mathrm{E}-18$ & LGLE & $1 \mathrm{E}-340$ & 0.23 \\
\hline SurfaceArea & $1 \mathrm{E}-340$ & $3.02 \mathrm{E}-22$ & RMS & $1 \mathrm{E}-340$ & 0.99 & IDMN & $1 \mathrm{E}-340$ & 0.62 & $\mathbf{R P}$ & $1 \mathrm{E}-340$ & 0.97 & SAHGLE & $1 \mathrm{E}-340$ & $2.00 \mathrm{E}-48$ & SDE & $1 \mathrm{E}-340$ & $4.03 \mathrm{E}-15$ \\
\hline SVR & $1 \mathrm{E}-340$ & $9.54 \mathrm{E}-07$ & Skewness & $1 \mathrm{E}-340$ & 0.99 & IDN & $1 \mathrm{E}-340$ & 0.93 & RV & $1 \mathrm{E}-340$ & 0.87 & SALGLE & $1 \mathrm{E}-340$ & 0.01 & SDHGLE & $1 \mathrm{E}-340$ & $1.73 \mathrm{E}-13$ \\
\hline \multirow[t]{11}{*}{ VoxelVolume } & $1 \mathrm{E}-340$ & $2.21 \mathrm{E}-05$ & TE & $1 \mathrm{E}-340$ & 0.96 & IMC1 & $1 \mathrm{E}-340$ & 0.58 & SRE & $1 \mathrm{E}-340$ & 0.97 & $\mathbf{Z E}$ & $1 \mathrm{E}-340$ & 0.88 & SDLGLE & $1 \mathrm{E}-340$ & 0.01 \\
\hline & & & Uniformity & $1 \mathrm{E}-340$ & 0.99 & IMC2 & $1 \mathrm{E}-340$ & 0.88 & SRHGLE & $1 \mathrm{E}-340$ & $1.36 \mathrm{E}-16$ & ZP & $1 \mathrm{E}-340$ & $1.16 \mathrm{E}-08$ & NGTDM_Busyness & $1 \mathrm{E}-340$ & 0.40 \\
\hline & & & Variance & $1 \mathrm{E}-340$ & 0.98 & IV & $1 \mathrm{E}-340$ & 0.20 & SRLGLE & $1 \mathrm{E}-340$ & 0.17 & $\mathbf{Z V}$ & $1 \mathrm{E}-340$ & $1.13 \mathrm{E}-19$ & NGTDM_Coarseness & $1 \mathrm{E}-340$ & 0.69 \\
\hline & & & & & & JA & $1 E-340$ & 0.13 & & & & & & & NGTDM_Complexity & $1 E-340$ & 3.17E-09 \\
\hline & & & & & & JEnergy & $1 \mathrm{E}-340$ & 1 & & & & & & & NGTDM_Contrast & $1 \mathrm{E}-340$ & 0.81 \\
\hline & & & & & & JEntropy & $1 \mathrm{E}-340$ & 0.99 & & & & & & & NGTDM_Strength & $1 \mathrm{E}-340$ & $4.31 \mathrm{E}-17$ \\
\hline & & & & & & MCC & $1 \mathrm{E}-340$ & 0.69 & & & & & & & & & \\
\hline & & & & & & MP & $1 \mathrm{E}-340$ & 0.99 & & & & & & & & & \\
\hline & & & & & & SA & $1 \mathrm{E}-340$ & 0.13 & & & & & & & & & \\
\hline & & & & & & SE & $1 \mathrm{E}-340$ & 0.99 & & & & & & & & & \\
\hline & & & & & & SS & $1 \mathrm{E}-340$ & 0.95 & & & & & & & & & \\
\hline
\end{tabular}


Supplemental Table 2. Classification performance indices for different feature selectors (FS) and classifiers in Strategy 1.

\begin{tabular}{|c|c|c|c|c|c|c|c|}
\hline FS & Classifier & AUC & 95\% CIs & Std & Acc & Sen & Spe \\
\hline \multirow{7}{*}{ ANOVA } & $\mathrm{RF}$ & 0.83 & [0.81-0.85] & 0.010 & 0.74 & 0.79 & 0.74 \\
\hline & $\mathrm{AB}$ & 0.81 & [0.79-0.83] & 0.011 & 0.73 & 0.74 & 0.73 \\
\hline & $\mathrm{AE}$ & 0.83 & [0.81-0.85] & 0.010 & 0.74 & 0.78 & 0.74 \\
\hline & LDA & 0.82 & [0.80-0.84] & 0.011 & 0.73 & 0.77 & 0.73 \\
\hline & LR & 0.82 & [0.80-0.84] & 0.010 & 0.75 & 0.77 & 0.75 \\
\hline & LASSO & 0.82 & {$[0.80-0.84]$} & 0.010 & 0.76 & 0.76 & 0.76 \\
\hline & NB & 0.80 & {$[0.78-0.82]$} & 0.011 & 0.73 & 0.75 & 0.73 \\
\hline \multirow{7}{*}{ KW } & $\mathrm{RF}$ & 0.83 & [0.81-0.85] & 0.010 & 0.74 & 0.79 & 0.74 \\
\hline & $\mathrm{AB}$ & 0.80 & {$[0.78-0.82]$} & 0.011 & 0.75 & 0.75 & 0.75 \\
\hline & $\mathrm{AE}$ & 0.83 & [0.81-0.85] & 0.010 & 0.76 & 0.76 & 0.76 \\
\hline & LDA & 0.82 & {$[0.80-0.84]$} & 0.011 & 0.74 & 0.77 & 0.74 \\
\hline & LR & 0.82 & [0.80-0.84] & 0.010 & 0.75 & 0.77 & 0.74 \\
\hline & LASSO & 0.83 & [0.81-0.84] & 0.010 & 0.75 & 0.77 & 0.75 \\
\hline & NB & 0.80 & {$[0.78-0.82]$} & 0.011 & 0.73 & 0.74 & 0.73 \\
\hline \multirow{7}{*}{ Relief } & $\mathrm{RF}$ & 0.84 & [0.82-0.85] & 0.010 & 0.76 & 0.77 & 0.76 \\
\hline & $\mathrm{AB}$ & 0.81 & {$[0.79-0.83]$} & 0.010 & 0.73 & 0.75 & 0.73 \\
\hline & $\mathrm{AE}$ & 0.83 & [0.81-0.84] & 0.010 & 0.76 & 0.76 & 0.76 \\
\hline & LDA & 0.82 & [0.79-0.84] & 0.011 & 0.75 & 0.76 & 0.75 \\
\hline & LR & 0.82 & [0.80-0.84] & 0.010 & 0.73 & 0.77 & 0.73 \\
\hline & LASSO & 0.83 & [0.81-0.85] & 0.010 & 0.76 & 0.76 & 0.76 \\
\hline & NB & 0.79 & {$[0.77-0.82]$} & 0.011 & 0.76 & 0.70 & 0.77 \\
\hline \multirow{7}{*}{ RFE } & $\mathrm{RF}$ & 0.84 & {$[0.82-0.85]$} & 0.010 & 0.76 & 0.78 & 0.76 \\
\hline & $\mathrm{AB}$ & 0.81 & {$[0.79-0.83]$} & 0.010 & 0.73 & 0.75 & 0.73 \\
\hline & $\mathrm{AE}$ & 0.83 & [0.81-0.85] & 0.010 & 0.74 & 0.78 & 0.74 \\
\hline & LDA & 0.82 & {$[0.79-0.84]$} & 0.011 & 0.74 & 0.77 & 0.74 \\
\hline & LR & 0.82 & {$[0.80-0.84]$} & 0.010 & 0.74 & 0.76 & 0.74 \\
\hline & LASSO & 0.83 & [0.81-0.85] & 0.010 & 0.75 & 0.77 & 0.75 \\
\hline & NB & 0.79 & {$[0.77-0.81]$} & 0.011 & 0.71 & 0.74 & 0.71 \\
\hline
\end{tabular}


Supplemental Table 3. Classification performance indices for different feature selectors (FS) and classifiers in Strategy 2.

\begin{tabular}{|c|c|c|c|c|c|c|c|}
\hline FS & Classifier & AUC & 95\% CIs & Std & Acc & Sen & Spe \\
\hline \multirow{7}{*}{ ANOVA } & $\mathrm{RF}$ & 0.84 & {$[0.82-0.86]$} & 0.011 & 0.76 & 0.78 & 0.76 \\
\hline & $\mathrm{AB}$ & 0.83 & [0.80-0.85] & 0.010 & 0.76 & 0.76 & 0.76 \\
\hline & $\mathrm{AE}$ & 0.84 & {$[0.82-0.86]$} & 0.010 & 0.75 & 0.79 & 0.75 \\
\hline & LDA & 0.81 & {$[0.79-0.84]$} & 0.011 & 0.74 & 0.76 & 0.74 \\
\hline & LR & 0.82 & [0.80-0.84] & 0.010 & 0.75 & 0.76 & 0.75 \\
\hline & LASSO & 0.83 & [0.81-0.85] & 0.010 & 0.75 & 0.77 & 0.75 \\
\hline & NB & 0.81 & {$[0.78-0.83]$} & 0.011 & 0.73 & 0.77 & 0.73 \\
\hline \multirow{7}{*}{ KW } & RF & 0.84 & {$[0.82-0.86]$} & 0.010 & 0.76 & 0.81 & 0.76 \\
\hline & $\mathrm{AB}$ & 0.81 & {$[0.79-0.83]$} & 0.011 & 0.73 & 0.76 & 0.73 \\
\hline & $\mathrm{AE}$ & 0.82 & [0.80-0.84] & 0.010 & 0.74 & 0.77 & 0.74 \\
\hline & LDA & 0.82 & [0.80-0.84] & 0.011 & 0.75 & 0.77 & 0.75 \\
\hline & LR & 0.83 & {$[0.80-0.85]$} & 0.010 & 0.76 & 0.76 & 0.76 \\
\hline & LASSO & 0.83 & {$[0.80-0.85]$} & 0.011 & 0.76 & 0.76 & 0.76 \\
\hline & NB & 0.81 & [0.78-0.83] & 0.011 & 0.74 & 0.77 & 0.73 \\
\hline \multirow{7}{*}{ Relief } & RF & 0.84 & {$[0.82-0.86]$} & 0.011 & 0.77 & 0.79 & 0.76 \\
\hline & $\mathrm{AB}$ & 0.83 & [0.81-0.85] & 0.010 & 0.75 & 0.78 & 0.74 \\
\hline & $\mathrm{AE}$ & 0.84 & [0.82-0.86] & 0.010 & 0.75 & 0.78 & 0.75 \\
\hline & LDA & 0.82 & {$[0.80-0.84]$} & 0.011 & 0.75 & 0.78 & 0.74 \\
\hline & LR & 0.82 & {$[0.79-0.84]$} & 0.011 & 0.74 & 0.76 & 0.74 \\
\hline & LASSO & 0.83 & [0.81-0.85] & 0.010 & 0.76 & 0.79 & 0.75 \\
\hline & NB & 0.80 & {$[0.78-0.82]$} & 0.012 & 0.75 & 0.73 & 0.75 \\
\hline \multirow{7}{*}{ RFE } & $\mathrm{RF}$ & 0.84 & [0.82-0.86] & 0.011 & 0.75 & 0.79 & 0.75 \\
\hline & $\mathrm{AB}$ & 0.83 & [0.81-0.85] & 0.010 & 0.74 & 0.79 & 0.74 \\
\hline & $\mathrm{AE}$ & 0.83 & [0.81-0.85] & 0.010 & 0.76 & 0.77 & 0.76 \\
\hline & LDA & 0.82 & {$[0.80-0.84]$} & 0.011 & 0.74 & 0.77 & 0.74 \\
\hline & LR & 0.83 & {$[0.81-0.85]$} & 0.010 & 0.75 & 0.77 & 0.75 \\
\hline & LASSO & 0.83 & [0.81-0.85] & 0.010 & 0.75 & 0.76 & 0.75 \\
\hline & NB & 0.80 & {$[0.78-0.83]$} & 0.012 & 0.75 & 0.75 & 0.74 \\
\hline
\end{tabular}


Supplemental Table 4. Classification performance indices for different feature selectors (FS) and classifiers in Strategy 3.

\begin{tabular}{|c|c|c|c|c|c|c|c|}
\hline FS & Classifie & AUC & 95\% CIs & Std & Acc & Sen & Spe \\
\hline \multirow{7}{*}{ ANOVA } & RF & 0.83 & {$[0.81-0.85]$} & 0.010 & 0.73 & 0.81 & 0.72 \\
\hline & $\mathrm{AB}$ & 0.81 & [0.79-0.83] & 0.010 & 0.69 & 0.81 & 0.68 \\
\hline & $\mathrm{AE}$ & 0.83 & [0.81-0.85] & 0.010 & 0.73 & 0.80 & 0.72 \\
\hline & LDA & 0.81 & [0.79-0.83] & 0.012 & 0.75 & 0.75 & 0.75 \\
\hline & LR & 0.82 & {$[0.8-0.84]$} & 0.010 & 0.71 & 0.81 & 0.70 \\
\hline & LASSO & 0.83 & [0.81-0.85] & 0.010 & 0.73 & 0.80 & 0.72 \\
\hline & NB & 0.80 & {$[0.77-0.82]$} & 0.011 & 0.77 & 0.71 & 0.78 \\
\hline \multirow{7}{*}{ KW } & $\mathrm{RF}$ & 0.81 & [0.79-0.83] & 0.011 & 0.73 & 0.75 & 0.72 \\
\hline & $\mathrm{AB}$ & 0.80 & {$[0.77-0.82]$} & 0.011 & 0.73 & 0.75 & 0.72 \\
\hline & $\mathrm{AE}$ & 0.80 & {$[0.78-0.82]$} & 0.011 & 0.71 & 0.77 & 0.70 \\
\hline & LDA & 0.82 & {$[0.80-0.84]$} & 0.010 & 0.73 & 0.80 & 0.72 \\
\hline & LR & 0.81 & [0.79-0.83] & 0.011 & 0.75 & 0.76 & 0.74 \\
\hline & LASSO & 0.83 & {$[0.81-0.85]$} & 0.010 & 0.74 & 0.76 & 0.74 \\
\hline & NB & 0.79 & {$[0.77-0.81]$} & 0.011 & 0.76 & 0.71 & 0.77 \\
\hline \multirow{7}{*}{ Relief } & $\mathrm{RF}$ & 0.83 & {$[0.81-0.85]$} & 0.010 & 0.76 & 0.77 & 0.76 \\
\hline & $\mathrm{AB}$ & 0.81 & [0.79-0.83] & 0.010 & 0.75 & 0.73 & 0.75 \\
\hline & $\mathrm{AE}$ & 0.83 & [0.82-0.85] & 0.009 & 0.78 & 0.74 & 0.79 \\
\hline & LDA & 0.81 & [0.79-0.83] & 0.011 & 0.77 & 0.75 & 0.77 \\
\hline & LR & 0.81 & [0.79-0.83] & 0.011 & 0.73 & 0.76 & 0.73 \\
\hline & LASSO & 0.82 & {$[0.80-0.84]$} & 0.011 & 0.76 & 0.76 & 0.75 \\
\hline & NB & 0.79 & [0.76-0.81] & 0.012 & 0.74 & 0.72 & 0.74 \\
\hline \multirow{7}{*}{ RFE } & $\mathrm{RF}$ & 0.83 & {$[0.82-0.85]$} & 0.010 & 0.75 & 0.77 & 0.74 \\
\hline & $\mathrm{AB}$ & 0.81 & [0.79-0.83] & 0.011 & 0.75 & 0.74 & 0.75 \\
\hline & $\mathrm{AE}$ & 0.83 & {$[0.81-0.85]$} & 0.010 & 0.76 & 0.77 & 0.76 \\
\hline & LDA & 0.81 & [0.79-0.83] & 0.011 & 0.75 & 0.75 & 0.75 \\
\hline & LR & 0.82 & {$[0.79-0.84]$} & 0.011 & 0.74 & 0.76 & 0.74 \\
\hline & LASSO & 0.82 & [0.80-0.84] & 0.010 & 0.76 & 0.75 & 0.77 \\
\hline & NB & 0.79 & {$[0.77-0.81]$} & 0.011 & 0.72 & 0.73 & 0.72 \\
\hline
\end{tabular}


Supplemental Table 5. Classification performance indices for different feature selectors (FS) and classifiers in Strategy 4.

\begin{tabular}{|c|c|c|c|c|c|c|c|}
\hline FS & Classifier & AUC & 95\% CIs & Std & Acc & Sen & Spe \\
\hline \multirow{7}{*}{ ANOVA } & $\mathrm{RF}$ & 0.84 & {$[0.82-0.85]$} & 0.010 & 0.7477 & 0.79 & 0.74 \\
\hline & $\mathrm{AB}$ & 0.82 & {$[0.80-0.84]$} & 0.010 & 0.7483 & 0.75 & 0.75 \\
\hline & $\mathrm{AE}$ & 0.84 & [0.82-0.86] & 0.009 & 0.7354 & 0.81 & 0.72 \\
\hline & LDA & 0.82 & [0.80-0.84] & 0.011 & 0.7458 & 0.78 & 0.74 \\
\hline & LR & 0.83 & [0.81-0.85] & 0.010 & 0.7285 & 0.80 & 0.72 \\
\hline & LASSO & 0.83 & [0.81-0.85] & 0.010 & 0.751 & 0.79 & 0.75 \\
\hline & NB & 0.80 & {$[0.78-0.82]$} & 0.011 & 0.7389 & 0.75 & 0.74 \\
\hline \multirow{7}{*}{ KW } & $\mathrm{RF}$ & 0.84 & [0.82-0.85] & 0.010 & 0.7491 & 0.79 & 0.74 \\
\hline & $\mathrm{AB}$ & 0.82 & {$[0.80-0.84]$} & 0.010 & 0.7497 & 0.77 & 0.75 \\
\hline & $\mathrm{AE}$ & 0.84 & {$[0.82-0.86]$} & 0.009 & 0.7684 & 0.77 & 0.77 \\
\hline & LDA & 0.82 & [0.80-0.84] & 0.011 & 0.7461 & 0.78 & 0.74 \\
\hline & LR & 0.82 & {$[0.80-0.84]$} & 0.010 & 0.7461 & 0.77 & 0.74 \\
\hline & LASSO & 0.83 & [0.81-0.85] & 0.010 & 0.7574 & 0.78 & 0.75 \\
\hline & NB & 0.80 & [0.78-0.82] & 0.011 & 0.734 & 0.75 & 0.73 \\
\hline \multirow{7}{*}{ Relief } & $\mathrm{RF}$ & 0.83 & [0.81-0.85] & 0.010 & 0.7475 & 0.79 & 0.74 \\
\hline & $\mathrm{AB}$ & 0.81 & {$[0.79-0.83]$} & 0.010 & 0.7494 & 0.75 & 0.75 \\
\hline & $\mathrm{AE}$ & 0.83 & [0.81-0.85] & 0.010 & 0.7494 & 0.80 & 0.74 \\
\hline & LDA & 0.82 & [0.80-0.84] & 0.011 & 0.7428 & 0.79 & 0.74 \\
\hline & LR & 0.82 & [0.80-0.84] & 0.010 & 0.7409 & 0.78 & 0.73 \\
\hline & LASSO & 0.83 & [0.81-0.85] & 0.010 & 0.7466 & 0.81 & 0.74 \\
\hline & NB & 0.79 & [0.76-0.81] & 0.011 & 0.7494 & 0.71 & 0.76 \\
\hline \multirow{7}{*}{ RFE } & $\mathrm{RF}$ & 0.84 & {$[0.82-0.85]$} & 0.010 & 0.7384 & 0.80 & 0.73 \\
\hline & $\mathrm{AB}$ & 0.83 & [0.81-0.84] & 0.010 & 0.7422 & 0.78 & 0.74 \\
\hline & $\mathrm{AE}$ & 0.83 & [0.81-0.85] & 0.010 & 0.7618 & 0.79 & 0.76 \\
\hline & LDA & 0.82 & {$[0.80-0.84]$} & 0.011 & 0.7409 & 0.78 & 0.74 \\
\hline & LR & 0.82 & [0.80-0.84] & 0.010 & 0.7329 & 0.79 & 0.72 \\
\hline & LASSO & 0.83 & [0.81-0.85] & 0.010 & 0.7483 & 0.78 & 0.74 \\
\hline & NB & 0.79 & {$[0.77-0.81]$} & 0.011 & 0.7359 & 0.74 & 0.73 \\
\hline
\end{tabular}


Supplemental Table 6. Classification performance indices for different feature selectors (FS) and classifiers in Strategy 5.

\begin{tabular}{|c|c|c|c|c|c|c|c|}
\hline FS & Classifier & AUC & $95 \%$ CIs & Std & Acc & Sen & Spe \\
\hline \multirow{7}{*}{ ANOVA } & RF & 0.83 & [0.81-0.85] & 0.010 & 0.73 & 0.77 & 0.73 \\
\hline & $\mathrm{AB}$ & 0.79 & {$[0.76-0.81]$} & 0.012 & 0.74 & 0.72 & 0.75 \\
\hline & $\mathrm{AE}$ & 0.82 & {$[0.79-0.84]$} & 0.011 & 0.76 & 0.75 & 0.77 \\
\hline & LDA & 0.77 & [0.74-0.79] & 0.013 & 0.72 & 0.71 & 0.72 \\
\hline & LR & 0.77 & {$[0.75-0.80]$} & 0.012 & 0.72 & 0.73 & 0.72 \\
\hline & LASSO & 0.77 & {$[0.75-0.80]$} & 0.012 & 0.73 & 0.72 & 0.73 \\
\hline & NB & 0.75 & [0.72-0.77] & 0.013 & 0.72 & 0.70 & 0.72 \\
\hline \multirow{7}{*}{$\mathbf{K W}$} & $\mathrm{RF}$ & 0.82 & [0.80-0.84] & 0.010 & 0.75 & 0.75 & 0.75 \\
\hline & $\mathrm{AB}$ & 0.79 & {$[0.76-0.81]$} & 0.012 & 0.73 & 0.72 & 0.73 \\
\hline & $\mathrm{AE}$ & 0.81 & [0.79-0.83] & 0.011 & 0.75 & 0.76 & 0.75 \\
\hline & LDA & 0.77 & [0.74-0.79] & 0.013 & 0.72 & 0.71 & 0.72 \\
\hline & LR & 0.77 & [0.75-0.80] & 0.012 & 0.71 & 0.73 & 0.71 \\
\hline & LASSO & 0.77 & {$[0.75-0.80]$} & 0.012 & 0.72 & 0.73 & 0.72 \\
\hline & NB & 0.74 & [0.71-0.76] & 0.013 & 0.68 & 0.71 & 0.68 \\
\hline \multirow{7}{*}{ Relief } & $\mathrm{RF}$ & 0.83 & [0.81-0.85] & 0.010 & 0.74 & 0.77 & 0.74 \\
\hline & $\mathrm{AB}$ & 0.77 & [0.74-0.79] & 0.012 & 0.71 & 0.72 & 0.71 \\
\hline & $\mathrm{AE}$ & 0.79 & {$[0.77-0.81]$} & 0.012 & 0.72 & 0.74 & 0.71 \\
\hline & LDA & 0.76 & [0.74-0.78] & 0.012 & 0.71 & 0.71 & 0.71 \\
\hline & LR & 0.77 & {$[0.75-0.79]$} & 0.012 & 0.71 & 0.74 & 0.71 \\
\hline & LASSO & 0.77 & {$[0.75-0.80]$} & 0.012 & 0.71 & 0.74 & 0.71 \\
\hline & NB & 0.75 & {$[0.72-0.77]$} & 0.013 & 0.66 & 0.75 & 0.65 \\
\hline \multirow{7}{*}{ RFE } & $\mathrm{RF}$ & 0.83 & [0.81-0.85] & 0.010 & 0.74 & 0.76 & 0.74 \\
\hline & $\mathrm{AB}$ & 0.78 & {$[0.76-0.80]$} & 0.012 & 0.71 & 0.74 & 0.70 \\
\hline & $\mathrm{AE}$ & 0.82 & [0.80-0.84] & 0.011 & 0.75 & 0.75 & 0.75 \\
\hline & LDA & 0.77 & [0.74-0.79] & 0.013 & 0.72 & 0.71 & 0.72 \\
\hline & LR & 0.77 & {$[0.75-0.80]$} & 0.012 & 0.71 & 0.73 & 0.71 \\
\hline & LASSO & 0.77 & {$[0.75-0.80]$} & 0.012 & 0.73 & 0.72 & 0.73 \\
\hline & NB & 0.75 & {$[0.72-0.77]$} & 0.013 & 0.70 & 0.72 & 0.69 \\
\hline
\end{tabular}


Supplemental Table 7. Classification performance indices of different feature selector and classifiers in Strategy 6.

\begin{tabular}{|c|c|c|c|c|c|c|c|}
\hline FS & Classifier & $\mathbf{A U C}$ & 95\% CIs & Std & Acc & Sen & Spe \\
\hline \multirow{7}{*}{ ANOVA } & RF & 0.82 & [0.81-0.84] & 0.010 & 0.76 & 0.76 & 0.77 \\
\hline & $\mathrm{AB}$ & 0.78 & {$[0.76-0.81]$} & 0.012 & 0.73 & 0.75 & 0.73 \\
\hline & $\mathrm{AE}$ & 0.82 & [0.80-0.84] & 0.010 & 0.73 & 0.77 & 0.73 \\
\hline & LDA & 0.77 & [0.74-0.79] & 0.012 & 0.72 & 0.72 & 0.72 \\
\hline & LR & 0.78 & {$[0.75-0.80]$} & 0.012 & 0.72 & 0.74 & 0.72 \\
\hline & LASSO & 0.78 & {$[0.75-0.80]$} & 0.013 & 0.74 & 0.74 & 0.74 \\
\hline & NB & 0.76 & {$[0.73-0.78]$} & 0.012 & 0.71 & 0.72 & 0.71 \\
\hline \multirow{7}{*}{ KW } & RF & 0.82 & {$[0.80-0.84]$} & 0.010 & 0.74 & 0.79 & 0.73 \\
\hline & $\mathrm{AB}$ & 0.79 & {$[0.76-0.81]$} & 0.012 & 0.72 & 0.75 & 0.71 \\
\hline & $\mathrm{AE}$ & 0.82 & [0.79-0.84] & 0.011 & 0.73 & 0.78 & 0.73 \\
\hline & LDA & 0.77 & [0.74-0.79] & 0.013 & 0.71 & 0.73 & 0.71 \\
\hline & LR & 0.78 & {$[0.75-0.80]$} & 0.012 & 0.72 & 0.75 & 0.71 \\
\hline & LASSO & 0.78 & {$[0.75-0.80]$} & 0.013 & 0.72 & 0.75 & 0.71 \\
\hline & NB & 0.76 & {$[0.74-0.78]$} & 0.012 & 0.71 & 0.72 & 0.71 \\
\hline \multirow{7}{*}{ Relief } & $\mathrm{RF}$ & 0.83 & [0.81-0.84] & 0.010 & 0.73 & 0.79 & 0.72 \\
\hline & $\mathrm{AB}$ & 0.78 & {$[0.76-0.81]$} & 0.012 & 0.72 & 0.72 & 0.72 \\
\hline & $\mathrm{AE}$ & 0.81 & {$[0.79-0.83]$} & 0.011 & 0.74 & 0.75 & 0.73 \\
\hline & LDA & 0.77 & {$[0.75-0.80]$} & 0.013 & 0.72 & 0.74 & 0.72 \\
\hline & LR & 0.78 & {$[0.75-0.80]$} & 0.012 & 0.72 & 0.73 & 0.72 \\
\hline & LASSO & 0.78 & {$[0.75-0.80]$} & 0.013 & 0.72 & 0.74 & 0.71 \\
\hline & NB & 0.75 & {$[0.73-0.78]$} & 0.012 & 0.67 & 0.75 & 0.66 \\
\hline \multirow{7}{*}{ RFE } & $\mathrm{RF}$ & 0.83 & [0.80-0.84] & 0.010 & 0.75 & 0.79 & 0.74 \\
\hline & $\mathrm{AB}$ & 0.79 & {$[0.76-0.81]$} & 0.012 & 0.73 & 0.74 & 0.73 \\
\hline & $\mathrm{AE}$ & 0.82 & {$[0.80-0.84]$} & 0.011 & 0.79 & 0.73 & 0.80 \\
\hline & LDA & 0.76 & {$[0.74-0.79]$} & 0.013 & 0.72 & 0.73 & 0.72 \\
\hline & LR & 0.78 & {$[0.75-0.80]$} & 0.012 & 0.71 & 0.75 & 0.71 \\
\hline & LASSO & 0.78 & {$[0.75-0.80]$} & 0.013 & 0.71 & 0.74 & 0.71 \\
\hline & NB & 0.76 & {$[0.74-0.78]$} & 0.012 & 0.71 & 0.71 & 0.71 \\
\hline
\end{tabular}


Supplemental Table 8. Classification performance indices of different feature selector and classifiers in Strategy 7.

\begin{tabular}{|c|c|c|c|c|c|c|c|}
\hline FS & Classifier & AUC & 95\% CIs & Std & Acc & Sen & Spe \\
\hline \multirow{7}{*}{ ANOVA } & $\mathrm{RF}$ & 0.78 & {$[0.76-0.80]$} & 0.011 & 0.73 & 0.71 & 0.73 \\
\hline & $\mathrm{AB}$ & 0.75 & {$[0.72-0.77]$} & 0.012 & 0.67 & 0.73 & 0.66 \\
\hline & $\mathrm{AE}$ & 0.78 & [0.76-0.80] & 0.011 & 0.73 & 0.70 & 0.73 \\
\hline & LDA & 0.76 & {$[0.73-0.78]$} & 0.012 & 0.72 & 0.70 & 0.72 \\
\hline & LR & 0.76 & {$[0.74-0.78]$} & 0.012 & 0.71 & 0.71 & 0.71 \\
\hline & LASSO & 0.76 & {$[0.74-0.79]$} & 0.012 & 0.71 & 0.72 & 0.71 \\
\hline & NB & 0.74 & {$[0.71-0.76]$} & 0.013 & 0.70 & 0.70 & 0.70 \\
\hline \multirow{7}{*}{ KW } & $\mathrm{RF}$ & 0.78 & {$[0.76-0.80]$} & 0.011 & 0.72 & 0.72 & 0.72 \\
\hline & $\mathrm{AB}$ & 0.76 & {$[0.73-0.78]$} & 0.012 & 0.71 & 0.70 & 0.71 \\
\hline & $\mathrm{AE}$ & 0.77 & {$[0.75-0.80]$} & 0.012 & 0.74 & 0.70 & 0.74 \\
\hline & LDA & 0.76 & {$[0.74-0.79]$} & 0.012 & 0.71 & 0.70 & 0.71 \\
\hline & LR & 0.76 & {$[0.74-0.78]$} & 0.012 & 0.75 & 0.67 & 0.76 \\
\hline & LASSO & 0.77 & {$[0.74-0.79]$} & 0.012 & 0.71 & 0.71 & 0.71 \\
\hline & NB & 0.74 & {$[0.72-0.77]$} & 0.013 & 0.74 & 0.65 & 0.75 \\
\hline \multirow{7}{*}{ Relief } & $\mathrm{RF}$ & 0.78 & {$[0.76-0.80]$} & 0.011 & 0.72 & 0.73 & 0.72 \\
\hline & $\mathrm{AB}$ & 0.74 & {$[0.72-0.76]$} & 0.012 & 0.68 & 0.72 & 0.67 \\
\hline & $\mathrm{AE}$ & 0.76 & {$[0.74-0.79]$} & 0.012 & 0.71 & 0.71 & 0.71 \\
\hline & LDA & 0.75 & {$[0.73-0.78]$} & 0.012 & 0.70 & 0.69 & 0.71 \\
\hline & LR & 0.76 & {$[0.74-0.78]$} & 0.012 & 0.73 & 0.67 & 0.74 \\
\hline & LASSO & 0.77 & {$[0.74-0.79]$} & 0.012 & 0.68 & 0.74 & 0.68 \\
\hline & NB & 0.73 & {$[0.70-0.75]$} & 0.013 & 0.72 & 0.67 & 0.72 \\
\hline \multirow{7}{*}{ RFE } & $\mathrm{RF}$ & 0.79 & {$[0.76-0.81]$} & 0.011 & 0.71 & 0.73 & 0.71 \\
\hline & $\mathrm{AB}$ & 0.75 & {$[0.72-0.77]$} & 0.012 & 0.68 & 0.73 & 0.67 \\
\hline & $\mathrm{AE}$ & 0.77 & {$[0.75-0.79]$} & 0.012 & 0.71 & 0.73 & 0.70 \\
\hline & LDA & 0.76 & [0.73-0.78] & 0.012 & 0.71 & 0.71 & 0.70 \\
\hline & LR & 0.76 & {$[0.74-0.79]$} & 0.012 & 0.72 & 0.70 & 0.73 \\
\hline & LASSO & 0.77 & {$[0.74-0.79]$} & 0.012 & 0.71 & 0.71 & 0.71 \\
\hline & NB & 0.74 & {$[0.72-0.77]$} & 0.013 & 0.72 & 0.67 & 0.73 \\
\hline
\end{tabular}


Supplemental Table 9. Classification performance indices of different feature selector and classifiers in Strategy 8.

\begin{tabular}{|c|c|c|c|c|c|}
\hline FS & Classifier & AUC & Acc & Sen & Spe \\
\hline \multirow{7}{*}{ ANOVA } & $\mathrm{RF}$ & $0.67 \pm 0.072$ & $0.61 \pm 0.085$ & $0.67 \pm 0.058$ & $0.6 \pm 0.096$ \\
\hline & $\mathrm{AB}$ & $0.69 \pm 0.064$ & $0.65 \pm 0.059$ & $0.66 \pm 0.059$ & $0.64 \pm 0.065$ \\
\hline & $\mathrm{AE}$ & $0.72 \pm 0.073$ & $0.66 \pm 0.068$ & $0.7 \pm 0.069$ & $0.65 \pm 0.077$ \\
\hline & LDA & $0.71 \pm 0.062$ & $0.66 \pm 0.058$ & $0.69 \pm 0.037$ & $0.66 \pm 0.063$ \\
\hline & LR & $0.74 \pm 0.051$ & $0.68 \pm 0.047$ & $0.71 \pm 0.039$ & $0.67 \pm 0.051$ \\
\hline & LASSO & $0.74 \pm 0.051$ & $0.68 \pm 0.042$ & $0.71 \pm 0.041$ & $0.68 \pm 0.045$ \\
\hline & NB & $0.74 \pm 0.034$ & $0.69 \pm 0.03$ & $0.71 \pm 0.026$ & $0.69 \pm 0.033$ \\
\hline \multirow{7}{*}{ KW } & $\mathrm{RF}$ & $0.67 \pm 0.08$ & $0.6 \pm 0.09$ & $0.67 \pm 0.074$ & $0.59 \pm 0.1$ \\
\hline & $\mathrm{AB}$ & $0.68 \pm 0.079$ & $0.61 \pm 0.064$ & $0.69 \pm 0.063$ & $0.6 \pm 0.068$ \\
\hline & $\mathrm{AE}$ & $0.72 \pm 0.07$ & $0.63 \pm 0.097$ & $0.73 \pm 0.067$ & $0.62 \pm 0.11$ \\
\hline & LDA & $0.7 \pm 0.074$ & $0.65 \pm 0.071$ & $0.68 \pm 0.05$ & $0.64 \pm 0.079$ \\
\hline & LR & $0.72 \pm 0.066$ & $0.65 \pm 0.051$ & $0.71 \pm 0.037$ & $0.65 \pm 0.056$ \\
\hline & LASSO & $0.74 \pm 0.053$ & $0.67 \pm 0.043$ & $0.71 \pm 0.039$ & $0.67 \pm 0.046$ \\
\hline & NB & $0.74 \pm 0.059$ & $0.69 \pm 0.049$ & $0.7 \pm 0.043$ & $0.69 \pm 0.052$ \\
\hline \multirow{7}{*}{ Relief } & $\mathrm{RF}$ & $0.66 \pm 0.066$ & $0.59 \pm 0.069$ & $0.67 \pm 0.057$ & $0.58 \pm 0.077$ \\
\hline & $\mathrm{AB}$ & $0.67 \pm 0.062$ & $0.58 \pm 0.073$ & $0.7 \pm 0.079$ & $0.57 \pm 0.084$ \\
\hline & $\mathrm{AE}$ & $0.71 \pm 0.057$ & $0.62 \pm 0.076$ & $0.71 \pm 0.039$ & $0.61 \pm 0.088$ \\
\hline & LDA & $0.69 \pm 0.061$ & $0.63 \pm 0.05$ & $0.67 \pm 0.051$ & $0.63 \pm 0.052$ \\
\hline & LR & $0.71 \pm 0.063$ & $0.63 \pm 0.068$ & $0.71 \pm 0.043$ & $0.62 \pm 0.077$ \\
\hline & LASSO & $0.7 \pm 0.069$ & $0.62 \pm 0.064$ & $0.71 \pm 0.053$ & $0.61 \pm 0.07$ \\
\hline & NB & $0.64 \pm 0.087$ & $0.63 \pm 0.1$ & $0.61 \pm 0.12$ & $0.63 \pm 0.12$ \\
\hline \multirow{7}{*}{ RFE } & $\mathrm{RF}$ & $0.67 \pm 0.071$ & $0.6 \pm 0.064$ & $0.67 \pm 0.053$ & $0.6 \pm 0.069$ \\
\hline & $\mathrm{AB}$ & $0.68 \pm 0.049$ & $0.6 \pm 0.059$ & $0.71 \pm 0.058$ & $0.58 \pm 0.07$ \\
\hline & $\mathrm{AE}$ & $0.72 \pm 0.066$ & $0.63 \pm 0.079$ & $0.72 \pm 0.029$ & $0.62 \pm 0.089$ \\
\hline & LDA & $0.68 \pm 0.058$ & $0.61 \pm 0.06$ & $0.7 \pm 0.047$ & $0.6 \pm 0.069$ \\
\hline & LR & $0.7 \pm 0.062$ & $0.63 \pm 0.059$ & $0.7 \pm 0.056$ & $0.62 \pm 0.067$ \\
\hline & LASSO & $0.7 \pm 0.062$ & $0.62 \pm 0.056$ & $0.7 \pm 0.052$ & $0.61 \pm 0.063$ \\
\hline & NB & $0.67 \pm 0.064$ & $0.59 \pm 0.099$ & $0.72 \pm 0.081$ & $0.57 \pm 0.12$ \\
\hline
\end{tabular}


Supplemental Table 10. Classification performance indices of different feature selector and classifiers in Strategy 9.

\begin{tabular}{|c|c|c|c|c|c|}
\hline FS & Classifier & AUC & Acc & Sen & Spe \\
\hline \multirow{7}{*}{ ANOVA } & $\mathrm{RF}$ & $0.79 \pm 0.08$ & $0.74 \pm 0.079$ & $0.77 \pm 0.086$ & $0.73 \pm 0.078$ \\
\hline & $\mathrm{AB}$ & $0.79 \pm 0.08$ & $0.75 \pm 0.073$ & $0.77 \pm 0.099$ & $0.74 \pm 0.074$ \\
\hline & $\mathrm{AE}$ & $0.80 \pm 0.08$ & $0.77 \pm 0.075$ & $0.78 \pm 0.095$ & $0.76 \pm 0.078$ \\
\hline & LDA & $0.79 \pm 0.08$ & $0.76 \pm 0.072$ & $0.76 \pm 0.1$ & $0.75 \pm 0.075$ \\
\hline & LR & $0.80 \pm 0.08$ & $0.76 \pm 0.075$ & $0.77 \pm 0.11$ & $0.76 \pm 0.075$ \\
\hline & LASSO & $0.80 \pm 0.08$ & $0.75 \pm 0.076$ & $0.77 \pm 0.099$ & $0.75 \pm 0.076$ \\
\hline & NB & $0.78 \pm 0.08$ & $0.75 \pm 0.068$ & $0.74 \pm 0.11$ & $0.76 \pm 0.07$ \\
\hline \multirow{7}{*}{ KW } & $\mathrm{RF}$ & $0.79 \pm 0.08$ & $0.72 \pm 0.093$ & $0.78 \pm 0.11$ & $0.71 \pm 0.098$ \\
\hline & $\mathrm{AB}$ & $0.79 \pm 0.086$ & $0.74 \pm 0.088$ & $0.76 \pm 0.16$ & $0.74 \pm 0.11$ \\
\hline & $\mathrm{AE}$ & $0.81 \pm 0.083$ & $0.73 \pm 0.095$ & $0.81 \pm 0.13$ & $0.73 \pm 0.1$ \\
\hline & LDA & $0.80 \pm 0.084$ & $0.74 \pm 0.092$ & $0.77 \pm 0.13$ & $0.74 \pm 0.11$ \\
\hline & LR & $0.80 \pm 0.084$ & $0.76 \pm 0.085$ & $0.76 \pm 0.14$ & $0.76 \pm 0.097$ \\
\hline & LASSO & $0.80 \pm 0.084$ & $0.75 \pm 0.095$ & $0.77 \pm 0.14$ & $0.74 \pm 0.11$ \\
\hline & NB & $0.78 \pm 0.083$ & $0.76 \pm 0.07$ & $0.75 \pm 0.12$ & $0.76 \pm 0.074$ \\
\hline \multirow{7}{*}{ Relief } & $\mathrm{RF}$ & $0.79 \pm 0.086$ & $0.72 \pm 0.098$ & $0.79 \pm 0.12$ & $0.71 \pm 0.1$ \\
\hline & $\mathrm{AB}$ & $0.79 \pm 0.08$ & $0.71 \pm 0.092$ & $0.81 \pm 0.08$ & $0.69 \pm 0.1$ \\
\hline & $\mathrm{AE}$ & $0.8 \pm 0.087$ & $0.73 \pm 0.093$ & $0.8 \pm 0.11$ & $0.72 \pm 0.1$ \\
\hline & LDA & $0.8 \pm 0.084$ & $0.73 \pm 0.092$ & $0.8 \pm 0.11$ & $0.72 \pm 0.1$ \\
\hline & LR & $0.79 \pm 0.077$ & $0.74 \pm 0.083$ & $0.78 \pm 0.11$ & $0.74 \pm 0.09$ \\
\hline & LASSO & $0.8 \pm 0.086$ & $0.75 \pm 0.095$ & $0.77 \pm 0.13$ & $0.74 \pm 0.11$ \\
\hline & NB & $0.75 \pm 0.075$ & $0.73 \pm 0.065$ & $0.71 \pm 0.11$ & $0.74 \pm 0.074$ \\
\hline \multirow{7}{*}{ RFE } & $\mathrm{RF}$ & $0.79 \pm 0.082$ & $0.71 \pm 0.089$ & $0.8 \pm 0.099$ & $0.7 \pm 0.096$ \\
\hline & $\mathrm{AB}$ & $0.79 \pm 0.077$ & $0.72 \pm 0.073$ & $0.8 \pm 0.081$ & $0.7 \pm 0.08$ \\
\hline & $\mathrm{AE}$ & $0.81 \pm 0.082$ & $0.74 \pm 0.087$ & $0.8 \pm 0.13$ & $0.73 \pm 0.099$ \\
\hline & LDA & $0.79 \pm 0.089$ & $0.74 \pm 0.093$ & $0.78 \pm 0.11$ & $0.73 \pm 0.11$ \\
\hline & LR & $0.8 \pm 0.084$ & $0.75 \pm 0.09$ & $0.78 \pm 0.1$ & $0.75 \pm 0.092$ \\
\hline & LASSO & $0.8 \pm 0.082$ & $0.75 \pm 0.073$ & $0.76 \pm 0.17$ & $0.76 \pm 0.09$ \\
\hline & NB & $0.76 \pm 0.069$ & $0.74 \pm 0.048$ & $0.73 \pm 0.092$ & $0.74 \pm 0.052$ \\
\hline
\end{tabular}


Supplemental Table 11. Classification performance indices of different feature selector and classifiers in Strategy 10.

\begin{tabular}{|c|c|c|c|c|c|}
\hline FS & Classifier & AUC & Acc & Sen & Spe \\
\hline \multirow{7}{*}{ ANOVA } & $\mathrm{RF}$ & $0.82 \pm 0.095$ & $0.78 \pm 0.11$ & $0.82 \pm 0.097$ & $0.77 \pm 0.11$ \\
\hline & $\mathrm{AB}$ & $0.8 \pm 0.13$ & $0.77 \pm 0.11$ & $0.81 \pm 0.11$ & $0.76 \pm 0.12$ \\
\hline & $\mathrm{AE}$ & $0.79 \pm 0.11$ & $0.74 \pm 0.11$ & $0.83 \pm 0.11$ & $0.73 \pm 0.11$ \\
\hline & LDA & $0.82 \pm 0.1$ & $0.77 \pm 0.094$ & $0.83 \pm 0.11$ & $0.77 \pm 0.093$ \\
\hline & LR & $0.82 \pm 0.1$ & $0.78 \pm 0.093$ & $0.84 \pm 0.12$ & $0.77 \pm 0.094$ \\
\hline & LASSO & $0.81 \pm 0.099$ & $0.78 \pm 0.085$ & $0.82 \pm 0.12$ & $0.78 \pm 0.085$ \\
\hline & NB & $0.79 \pm 0.12$ & $0.76 \pm 0.12$ & $0.83 \pm 0.12$ & $0.75 \pm 0.13$ \\
\hline \multirow{7}{*}{$\mathbf{K W}$} & $\mathrm{RF}$ & $0.82 \pm 0.09$ & $0.76 \pm 0.11$ & $0.83 \pm 0.11$ & $0.75 \pm 0.12$ \\
\hline & $\mathrm{AB}$ & $0.8 \pm 0.14$ & $0.74 \pm 0.15$ & $0.84 \pm 0.14$ & $0.72 \pm 0.16$ \\
\hline & $\mathrm{AE}$ & $0.79 \pm 0.11$ & $0.73 \pm 0.14$ & $0.83 \pm 0.13$ & $0.72 \pm 0.16$ \\
\hline & LDA & $0.81 \pm 0.1$ & $0.75 \pm 0.1$ & $0.84 \pm 0.14$ & $0.74 \pm 0.11$ \\
\hline & LR & $0.81 \pm 0.097$ & $0.75 \pm 0.11$ & $0.85 \pm 0.11$ & $0.75 \pm 0.12$ \\
\hline & LASSO & $0.81 \pm 0.095$ & $0.76 \pm 0.1$ & $0.85 \pm 0.11$ & $0.75 \pm 0.11$ \\
\hline & NB & $0.79 \pm 0.11$ & $0.75 \pm 0.12$ & $0.83 \pm 0.15$ & $0.74 \pm 0.13$ \\
\hline \multirow{7}{*}{ Relief } & $\mathrm{RF}$ & $0.83 \pm 0.1$ & $0.75 \pm 0.12$ & $0.85 \pm 0.11$ & $0.74 \pm 0.13$ \\
\hline & $\mathrm{AB}$ & $0.77 \pm 0.14$ & $0.71 \pm 0.15$ & $0.84 \pm 0.13$ & $0.69 \pm 0.15$ \\
\hline & $\mathrm{AE}$ & $0.79 \pm 0.11$ & $0.74 \pm 0.11$ & $0.81 \pm 0.13$ & $0.72 \pm 0.12$ \\
\hline & LDA & $0.81 \pm 0.11$ & $0.74 \pm 0.1$ & $0.86 \pm 0.11$ & $0.72 \pm 0.11$ \\
\hline & LR & $0.81 \pm 0.11$ & $0.73 \pm 0.13$ & $0.86 \pm 0.13$ & $0.71 \pm 0.14$ \\
\hline & LASSO & $0.8 \pm 0.11$ & $0.73 \pm 0.12$ & $0.87 \pm 0.12$ & $0.71 \pm 0.13$ \\
\hline & NB & $0.79 \pm 0.12$ & $0.74 \pm 0.13$ & $0.83 \pm 0.12$ & $0.72 \pm 0.14$ \\
\hline \multirow{7}{*}{ RFE } & $\mathrm{RF}$ & $0.82 \pm 0.099$ & $0.72 \pm 0.13$ & $0.87 \pm 0.092$ & $0.7 \pm 0.13$ \\
\hline & $\mathrm{AB}$ & $0.8 \pm 0.14$ & $0.76 \pm 0.13$ & $0.84 \pm 0.1$ & $0.74 \pm 0.13$ \\
\hline & $\mathrm{AE}$ & $0.8 \pm 0.1$ & $0.74 \pm 0.1$ & $0.84 \pm 0.13$ & $0.72 \pm 0.11$ \\
\hline & LDA & $0.81 \pm 0.11$ & $0.73 \pm 0.11$ & $0.87 \pm 0.1$ & $0.71 \pm 0.12$ \\
\hline & LR & $0.8 \pm 0.12$ & $0.73 \pm 0.14$ & $0.85 \pm 0.15$ & $0.71 \pm 0.15$ \\
\hline & LASSO & $0.79 \pm 0.11$ & $0.73 \pm 0.13$ & $0.83 \pm 0.16$ & $0.72 \pm 0.14$ \\
\hline & NB & $0.79 \pm 0.12$ & $0.75 \pm 0.13$ & $0.83 \pm 0.13$ & $0.74 \pm 0.14$ \\
\hline
\end{tabular}


Supplemental Table 12. Classification performance indices of different feature selector and classifiers in Strategy 8.

\begin{tabular}{|c|c|c|c|c|c|c|c|c|}
\hline Center & FS & Classifier & AUC & $95 \%$ CIs & Std & Acc & Sen & Spe \\
\hline \multirow{28}{*}{ Center_01 } & \multirow{7}{*}{ ANOVA } & $\mathrm{AB}$ & 0.6 & {$[0.59-0.62]$} & 0.0075 & 0.58 & 0.59 & 0.58 \\
\hline & & $\mathrm{AE}$ & 0.77 & {$[0.75-0.78]$} & 0.0061 & 0.68 & 0.73 & 0.67 \\
\hline & & LASSO & 0.59 & {$[0.57-0.6]$} & 0.0073 & 0.58 & 0.59 & 0.58 \\
\hline & & LDA & 0.63 & {$[0.62-0.65]$} & 0.0067 & 0.61 & 0.66 & 0.6 \\
\hline & & LR & 0.58 & {$[0.57-0.6]$} & 0.0072 & 0.57 & 0.61 & 0.56 \\
\hline & & NB & 0.67 & [0.66-0.68] & 0.007 & 0.66 & 0.66 & 0.66 \\
\hline & & $\mathrm{RF}$ & 0.59 & {$[0.58-0.61]$} & 0.0075 & 0.52 & 0.66 & 0.5 \\
\hline & \multirow{7}{*}{ KW } & $\mathrm{AB}$ & 0.59 & {$[0.57-0.6]$} & 0.0076 & 0.55 & 0.64 & 0.54 \\
\hline & & $\mathrm{AE}$ & 0.65 & {$[0.64-0.66]$} & 0.007 & 0.63 & 0.65 & 0.63 \\
\hline & & LASSO & 0.61 & [0.6-0.63] & 0.0076 & 0.58 & 0.64 & 0.57 \\
\hline & & LDA & 0.62 & {$[0.61-0.64]$} & 0.0069 & 0.62 & 0.64 & 0.62 \\
\hline & & LR & 0.6 & {$[0.59-0.62]$} & 0.0076 & 0.57 & 0.65 & 0.56 \\
\hline & & NB & 0.66 & {$[0.65-0.67]$} & 0.0068 & 0.63 & 0.66 & 0.63 \\
\hline & & RF & 0.59 & {$[0.58-0.61]$} & 0.0077 & 0.55 & 0.62 & 0.54 \\
\hline & \multirow{7}{*}{ Relief } & $\mathrm{AB}$ & 0.59 & {$[0.57-0.6]$} & 0.0071 & 0.36 & 0.85 & 0.3 \\
\hline & & $\mathrm{AE}$ & 0.68 & {$[0.66-0.69]$} & 0.0067 & 0.63 & 0.65 & 0.63 \\
\hline & & LASSO & 0.64 & {$[0.62-0.65]$} & 0.0072 & 0.53 & 0.73 & 0.51 \\
\hline & & LDA & 0.6 & {$[0.58-0.61]$} & 0.0073 & 0.54 & 0.67 & 0.53 \\
\hline & & LR & 0.64 & {$[0.63-0.66]$} & 0.007 & 0.55 & 0.71 & 0.54 \\
\hline & & NB & 0.52 & {$[0.51-0.54]$} & 0.0076 & 0.53 & 0.54 & 0.53 \\
\hline & & $\mathrm{RF}$ & 0.59 & {$[0.57-0.6]$} & 0.0077 & 0.53 & 0.64 & 0.51 \\
\hline & \multirow{7}{*}{ RFE } & $\mathrm{AB}$ & 0.65 & {$[0.64-0.67]$} & 0.007 & 0.58 & 0.65 & 0.57 \\
\hline & & $\mathrm{AE}$ & 0.74 & {$[0.73-0.75]$} & 0.0068 & 0.65 & 0.73 & 0.64 \\
\hline & & LASSO & 0.55 & {$[0.53-0.56]$} & 0.0074 & 0.54 & 0.57 & 0.53 \\
\hline & & LDA & 0.58 & [0.56-0.59] & 0.0072 & 0.54 & 0.65 & 0.52 \\
\hline & & LR & 0.57 & {$[0.55-0.58]$} & 0.0073 & 0.57 & 0.57 & 0.57 \\
\hline & & NB & 0.59 & {$[0.58-0.61]$} & 0.0069 & 0.49 & 0.71 & 0.46 \\
\hline & & $\mathrm{RF}$ & 0.57 & {$[0.55-0.58]$} & 0.0078 & 0.52 & 0.59 & 0.51 \\
\hline \multirow{10}{*}{ Center_02 } & \multirow{7}{*}{ ANOVA } & $\mathrm{AB}$ & 0.74 & {$[0.73-0.75]$} & 0.0062 & 0.67 & 0.71 & 0.67 \\
\hline & & $\mathrm{AE}$ & 0.8 & {$[0.78-0.81]$} & 0.0061 & 0.74 & 0.74 & 0.74 \\
\hline & & LASSO & 0.77 & {$[0.75-0.78]$} & 0.0065 & 0.7 & 0.72 & 0.7 \\
\hline & & LDA & 0.79 & {$[0.77-0.8]$} & 0.0062 & 0.73 & 0.71 & 0.73 \\
\hline & & LR & 0.76 & {$[0.75-0.77]$} & 0.0062 & 0.68 & 0.72 & 0.67 \\
\hline & & NB & 0.79 & [0.78-0.8] & 0.0061 & 0.72 & 0.75 & 0.71 \\
\hline & & $\mathrm{RF}$ & 0.77 & {$[0.75-0.78]$} & 0.0065 & 0.71 & 0.72 & 0.71 \\
\hline & \multirow{3}{*}{$\mathrm{KW}$} & $\mathrm{AB}$ & 0.75 & {$[0.73-0.76]$} & 0.0063 & 0.68 & 0.71 & 0.67 \\
\hline & & $\mathrm{AE}$ & 0.79 & {$[0.78-0.8]$} & 0.0061 & 0.72 & 0.75 & 0.72 \\
\hline & & LASSO & 0.78 & {$[0.77-0.79]$} & 0.0065 & 0.73 & 0.74 & 0.73 \\
\hline
\end{tabular}




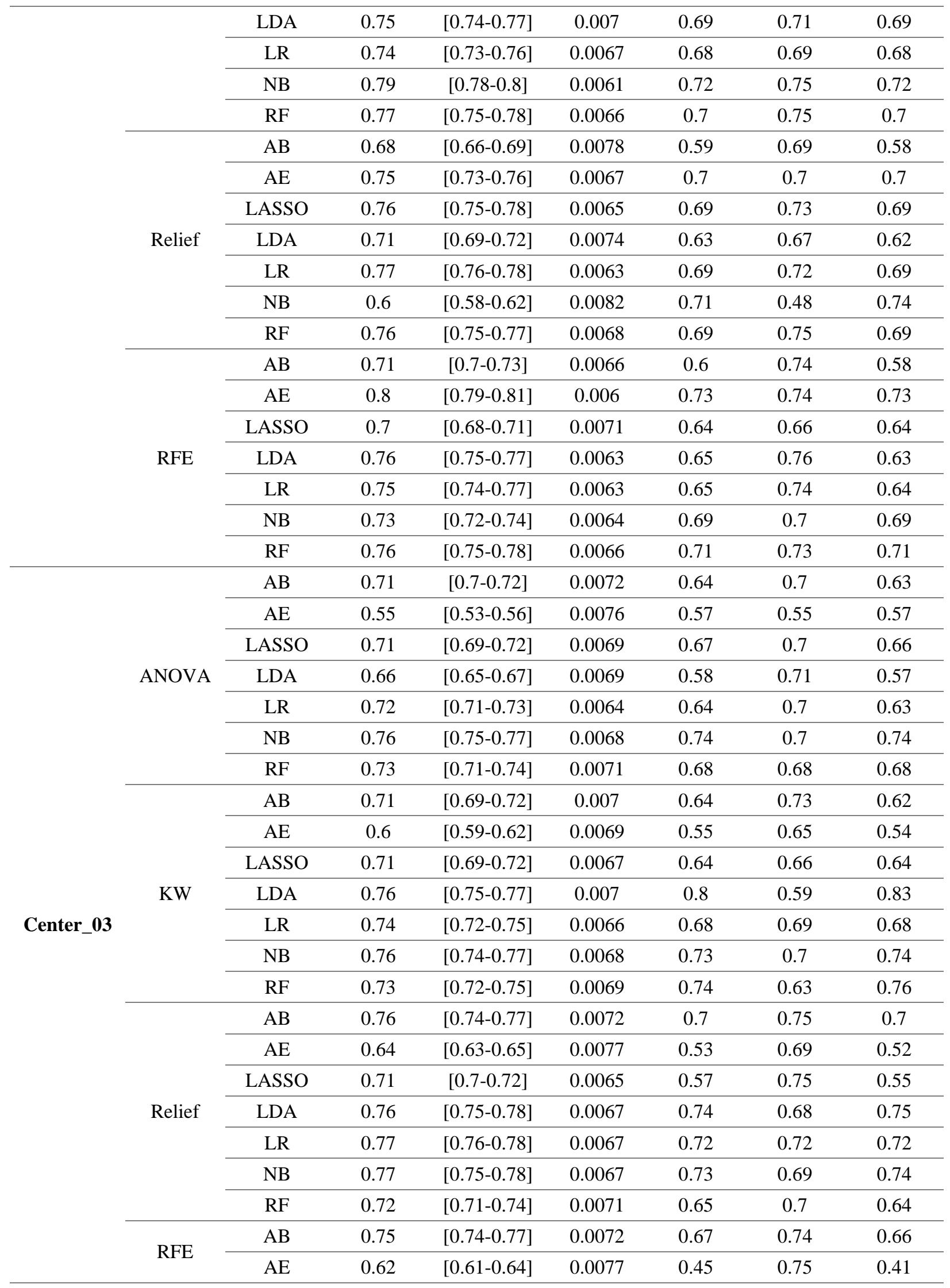




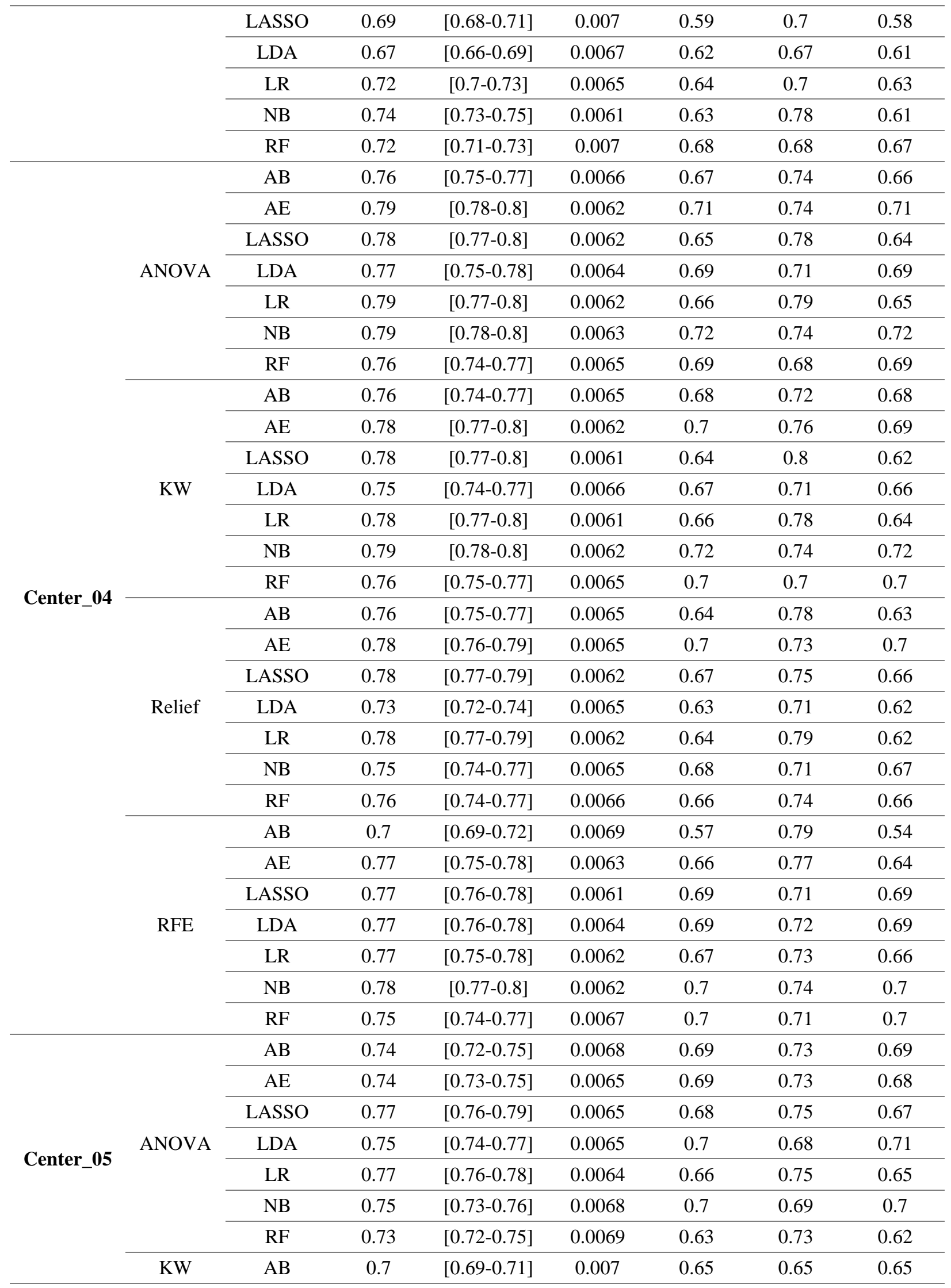




\begin{tabular}{|c|c|c|c|c|c|c|c|c|}
\hline & & $\mathrm{AE}$ & 0.74 & {$[0.72-0.75]$} & 0.0069 & 0.69 & 0.7 & 0.68 \\
\hline & & LASSO & 0.76 & {$[0.75-0.78]$} & 0.0065 & 0.68 & 0.71 & 0.68 \\
\hline & & LDA & 0.71 & {$[0.7-0.73]$} & 0.007 & 0.66 & 0.68 & 0.65 \\
\hline & & LR & 0.76 & {$[0.75-0.78]$} & 0.0065 & 0.7 & 0.7 & 0.7 \\
\hline & & NB & 0.76 & {$[0.75-0.78]$} & 0.0066 & 0.74 & 0.66 & 0.75 \\
\hline & & $\mathrm{RF}$ & 0.73 & {$[0.72-0.75]$} & 0.0069 & 0.63 & 0.73 & 0.62 \\
\hline & & $\mathrm{AB}$ & 0.72 & {$[0.7-0.73]$} & 0.0066 & 0.59 & 0.83 & 0.56 \\
\hline & & $\mathrm{AE}$ & 0.72 & {$[0.71-0.74]$} & 0.0068 & 0.66 & 0.7 & 0.65 \\
\hline & & LASSO & 0.72 & {$[0.71-0.73]$} & 0.0067 & 0.65 & 0.68 & 0.65 \\
\hline & Relief & LDA & 0.57 & {$[0.56-0.59]$} & 0.0082 & 0.57 & 0.57 & 0.57 \\
\hline & & LR & 0.72 & {$[0.7-0.73]$} & 0.0072 & 0.62 & 0.72 & 0.61 \\
\hline & & NB & 0.57 & {$[0.55-0.59]$} & 0.0086 & 0.8 & 0.3 & 0.86 \\
\hline & & $\mathrm{RF}$ & 0.75 & {$[0.74-0.77]$} & 0.0067 & 0.7 & 0.71 & 0.69 \\
\hline & & $\mathrm{AB}$ & 0.7 & [0.69-0.72] & 0.0067 & 0.57 & 0.81 & 0.54 \\
\hline & & $\mathrm{AE}$ & 0.75 & {$[0.74-0.76]$} & 0.0066 & 0.64 & 0.75 & 0.63 \\
\hline & & LASSO & 0.72 & {$[0.71-0.73]$} & 0.0068 & 0.64 & 0.69 & 0.63 \\
\hline & RFE & LDA & 0.69 & {$[0.67-0.7]$} & 0.0072 & 0.6 & 0.69 & 0.59 \\
\hline & & LR & 0.69 & {$[0.67-0.7]$} & 0.0074 & 0.64 & 0.65 & 0.63 \\
\hline & & NB & 0.61 & [0.59-0.62] & 0.0086 & 0.72 & 0.46 & 0.76 \\
\hline & & $\mathrm{RF}$ & 0.73 & {$[0.72-0.75]$} & 0.0066 & 0.62 & 0.75 & 0.6 \\
\hline & & $\mathrm{AB}$ & 0.67 & [0.66-0.69] & 0.0076 & 0.64 & 0.64 & 0.64 \\
\hline & & $\mathrm{AE}$ & 0.76 & {$[0.75-0.77]$} & 0.0064 & 0.68 & 0.71 & 0.68 \\
\hline & & LASSO & 0.7 & {$[0.69-0.72]$} & 0.0074 & 0.65 & 0.65 & 0.65 \\
\hline & ANOVA & LDA & 0.6 & {$[0.58-0.61]$} & 0.0078 & 0.58 & 0.56 & 0.58 \\
\hline & & LR & 0.74 & {$[0.73-0.76]$} & 0.0067 & 0.64 & 0.71 & 0.64 \\
\hline & & NB & 0.76 & {$[0.75-0.77]$} & 0.0064 & 0.67 & 0.73 & 0.66 \\
\hline & & $\mathrm{RF}$ & 0.58 & [0.56-0.59] & 0.0071 & 0.47 & 0.73 & 0.44 \\
\hline & & $\mathrm{AB}$ & 0.7 & {$[0.68-0.71]$} & 0.0072 & 0.6 & 0.77 & 0.58 \\
\hline & & $\mathrm{AE}$ & 0.75 & {$[0.74-0.77]$} & 0.0066 & 0.69 & 0.7 & 0.69 \\
\hline & & LASSO & 0.69 & {$[0.67-0.7]$} & 0.0078 & 0.64 & 0.65 & 0.64 \\
\hline Center_06 & KW & LDA & 0.56 & {$[0.54-0.57]$} & 0.0083 & 0.54 & 0.56 & 0.54 \\
\hline & & LR & 0.7 & {$[0.69-0.71]$} & 0.0074 & 0.63 & 0.67 & 0.62 \\
\hline & & NB & 0.75 & [0.74-0.77] & 0.0066 & 0.68 & 0.71 & 0.67 \\
\hline & & $\mathrm{RF}$ & 0.57 & {$[0.55-0.59]$} & 0.0079 & 0.53 & 0.58 & 0.52 \\
\hline & & $\mathrm{AB}$ & 0.68 & {$[0.67-0.69]$} & 0.0068 & 0.58 & 0.8 & 0.56 \\
\hline & & $\mathrm{AE}$ & 0.75 & {$[0.74-0.76]$} & 0.0068 & 0.67 & 0.71 & 0.66 \\
\hline & & LASSO & 0.72 & {$[0.71-0.74]$} & 0.0071 & 0.65 & 0.7 & 0.64 \\
\hline & Relief & LDA & 0.74 & {$[0.73-0.76]$} & 0.007 & 0.69 & 0.69 & 0.69 \\
\hline & & LR & 0.75 & {$[0.74-0.77]$} & 0.0066 & 0.67 & 0.71 & 0.66 \\
\hline & & NB & 0.72 & {$[0.7-0.73]$} & 0.0075 & 0.65 & 0.67 & 0.65 \\
\hline & & $\mathrm{RF}$ & 0.58 & [0.56-0.59] & 0.0076 & 0.54 & 0.61 & 0.53 \\
\hline
\end{tabular}




\begin{tabular}{|c|c|c|c|c|c|c|c|c|}
\hline & \multirow{7}{*}{ RFE } & $\mathrm{AB}$ & 0.65 & [0.64-0.66] & 0.0071 & 0.62 & 0.66 & 0.61 \\
\hline & & $\mathrm{AE}$ & 0.78 & {$[0.77-0.79]$} & 0.0062 & 0.72 & 0.72 & 0.72 \\
\hline & & LASSO & 0.72 & {$[0.7-0.73]$} & 0.0068 & 0.57 & 0.75 & 0.55 \\
\hline & & LDA & 0.69 & {$[0.67-0.7]$} & 0.0076 & 0.62 & 0.67 & 0.61 \\
\hline & & LR & 0.72 & {$[0.7-0.73]$} & 0.0068 & 0.53 & 0.79 & 0.5 \\
\hline & & NB & 0.73 & {$[0.72-0.75]$} & 0.0069 & 0.62 & 0.73 & 0.61 \\
\hline & & $\mathrm{RF}$ & 0.58 & [0.56-0.59] & 0.0071 & 0.47 & 0.73 & 0.44 \\
\hline \multirow{28}{*}{ Center_07 } & \multirow{7}{*}{ ANOVA } & $\mathrm{AB}$ & 0.75 & [0.73-0.76] & 0.0063 & 0.74 & 0.59 & 0.76 \\
\hline & & $\mathrm{AE}$ & 0.74 & {$[0.73-0.75]$} & 0.0068 & 0.66 & 0.73 & 0.66 \\
\hline & & LASSO & 0.78 & {$[0.77-0.8]$} & 0.0064 & 0.75 & 0.69 & 0.75 \\
\hline & & LDA & 0.76 & {$[0.75-0.77]$} & 0.0062 & 0.7 & 0.71 & 0.7 \\
\hline & & LR & 0.78 & {$[0.77-0.79]$} & 0.0063 & 0.7 & 0.74 & 0.7 \\
\hline & & NB & 0.78 & [0.77-0.79] & 0.0064 & 0.72 & 0.73 & 0.72 \\
\hline & & RF & 0.72 & {$[0.7-0.73]$} & 0.0079 & 0.71 & 0.64 & 0.71 \\
\hline & \multirow{7}{*}{ KW } & $\mathrm{AB}$ & 0.77 & {$[0.76-0.78]$} & 0.0063 & 0.69 & 0.73 & 0.68 \\
\hline & & $\mathrm{AE}$ & 0.71 & {$[0.7-0.73]$} & 0.0068 & 0.61 & 0.75 & 0.6 \\
\hline & & LASSO & 0.78 & {$[0.77-0.8]$} & 0.0062 & 0.71 & 0.72 & 0.7 \\
\hline & & LDA & 0.71 & [0.69-0.72] & 0.0071 & 0.65 & 0.66 & 0.65 \\
\hline & & LR & 0.76 & {$[0.75-0.77]$} & 0.0067 & 0.7 & 0.72 & 0.69 \\
\hline & & NB & 0.77 & {$[0.76-0.78]$} & 0.0065 & 0.72 & 0.72 & 0.72 \\
\hline & & $\mathrm{RF}$ & 0.76 & {$[0.75-0.77]$} & 0.0068 & 0.69 & 0.7 & 0.69 \\
\hline & \multirow{7}{*}{ Relief } & $\mathrm{AB}$ & 0.73 & {$[0.72-0.74]$} & 0.0065 & 0.66 & 0.66 & 0.66 \\
\hline & & $\mathrm{AE}$ & 0.71 & {$[0.7-0.73]$} & 0.0067 & 0.6 & 0.72 & 0.59 \\
\hline & & LASSO & 0.74 & {$[0.73-0.76]$} & 0.0065 & 0.66 & 0.71 & 0.66 \\
\hline & & LDA & 0.72 & {$[0.71-0.73]$} & 0.0067 & 0.66 & 0.68 & 0.66 \\
\hline & & LR & 0.75 & {$[0.73-0.76]$} & 0.0067 & 0.66 & 0.72 & 0.66 \\
\hline & & NB & 0.75 & {$[0.74-0.76]$} & 0.0062 & 0.7 & 0.71 & 0.7 \\
\hline & & $\mathrm{RF}$ & 0.68 & {$[0.67-0.7]$} & 0.0075 & 0.65 & 0.66 & 0.65 \\
\hline & \multirow{7}{*}{ RFE } & $\mathrm{AB}$ & 0.75 & {$[0.74-0.77]$} & 0.0063 & 0.67 & 0.73 & 0.66 \\
\hline & & $\mathrm{AE}$ & 0.62 & {$[0.61-0.63]$} & 0.0067 & 0.57 & 0.67 & 0.56 \\
\hline & & LASSO & 0.76 & [0.74-0.77] & 0.0064 & 0.68 & 0.72 & 0.68 \\
\hline & & LDA & 0.75 & {$[0.73-0.76]$} & 0.0065 & 0.65 & 0.76 & 0.63 \\
\hline & & LR & 0.77 & [0.76-0.78] & 0.0063 & 0.69 & 0.75 & 0.68 \\
\hline & & NB & 0.71 & {$[0.69-0.72]$} & 0.0064 & 0.64 & 0.72 & 0.63 \\
\hline & & $\mathrm{RF}$ & 0.69 & [0.68-0.71] & 0.0075 & 0.59 & 0.71 & 0.58 \\
\hline \multirow{6}{*}{ Center_08 } & \multirow{6}{*}{ ANOVA } & $\mathrm{AB}$ & 0.67 & {$[0.65-0.68]$} & 0.0077 & 0.61 & 0.66 & 0.61 \\
\hline & & $\mathrm{AB}$ & 0.67 & {$[0.65-0.68]$} & 0.0077 & 0.61 & 0.66 & 0.61 \\
\hline & & $\mathrm{AE}$ & 0.75 & {$[0.74-0.76]$} & 0.0061 & 0.7 & 0.7 & 0.7 \\
\hline & & $\mathrm{AE}$ & 0.75 & {$[0.74-0.76]$} & 0.0061 & 0.7 & 0.7 & 0.7 \\
\hline & & LASSO & 0.76 & {$[0.75-0.77]$} & 0.0061 & 0.68 & 0.73 & 0.67 \\
\hline & & LASSO & 0.76 & {$[0.75-0.77]$} & 0.0061 & 0.68 & 0.73 & 0.67 \\
\hline
\end{tabular}




\begin{tabular}{|c|c|c|c|c|c|c|c|c|}
\hline & & LDA & 0.73 & {$[0.71-0.74]$} & 0.0064 & 0.65 & 0.7 & 0.64 \\
\hline & \multirow{7}{*}{ KW } & LDA & 0.73 & {$[0.71-0.74]$} & 0.0064 & 0.65 & 0.7 & 0.64 \\
\hline & & LR & 0.75 & {$[0.74-0.77]$} & 0.0062 & 0.69 & 0.7 & 0.69 \\
\hline & & LR & 0.75 & {$[0.74-0.77]$} & 0.0062 & 0.69 & 0.7 & 0.69 \\
\hline & & NB & 0.78 & {$[0.77-0.79]$} & 0.006 & 0.72 & 0.74 & 0.72 \\
\hline & & NB & 0.78 & {$[0.77-0.79]$} & 0.006 & 0.72 & 0.74 & 0.72 \\
\hline & & $\mathrm{RF}$ & 0.66 & {$[0.65-0.68]$} & 0.0075 & 0.61 & 0.64 & 0.6 \\
\hline & & $\mathrm{RF}$ & 0.66 & {$[0.65-0.68]$} & 0.0075 & 0.61 & 0.64 & 0.6 \\
\hline & \multirow{7}{*}{ Relief } & $\mathrm{AB}$ & 0.63 & {$[0.62-0.65]$} & 0.0075 & 0.52 & 0.71 & 0.5 \\
\hline & & $\mathrm{AE}$ & 0.7 & {$[0.69-0.72]$} & 0.007 & 0.6 & 0.7 & 0.59 \\
\hline & & LASSO & 0.6 & {$[0.59-0.62]$} & 0.0074 & 0.54 & 0.66 & 0.53 \\
\hline & & LDA & 0.61 & {$[0.6-0.63]$} & 0.0078 & 0.58 & 0.59 & 0.57 \\
\hline & & LR & 0.62 & {$[0.61-0.64]$} & 0.0074 & 0.54 & 0.69 & 0.53 \\
\hline & & NB & 0.65 & {$[0.63-0.66]$} & 0.0075 & 0.63 & 0.63 & 0.63 \\
\hline & & $\mathrm{RF}$ & 0.64 & {$[0.63-0.66]$} & 0.0075 & 0.58 & 0.64 & 0.57 \\
\hline & \multirow{7}{*}{ RFE } & $\mathrm{AB}$ & 0.69 & {$[0.68-0.71]$} & 0.0072 & 0.63 & 0.69 & 0.62 \\
\hline & & $\mathrm{AE}$ & 0.76 & {$[0.75-0.77]$} & 0.0064 & 0.66 & 0.74 & 0.65 \\
\hline & & LASSO & 0.63 & {$[0.61-0.64]$} & 0.0083 & 0.58 & 0.64 & 0.57 \\
\hline & & LDA & 0.61 & {$[0.59-0.62]$} & 0.0075 & 0.57 & 0.63 & 0.57 \\
\hline & & LR & 0.64 & {$[0.62-0.65]$} & 0.0079 & 0.59 & 0.63 & 0.58 \\
\hline & & NB & 0.71 & {$[0.7-0.73]$} & 0.0062 & 0.64 & 0.73 & 0.63 \\
\hline & & $\mathrm{RF}$ & 0.65 & {$[0.64-0.67]$} & 0.0076 & 0.61 & 0.62 & 0.61 \\
\hline \multirow{19}{*}{ Center_09 } & \multirow{7}{*}{ ANOVA } & $\mathrm{AB}$ & 0.57 & {$[0.56-0.58]$} & 0.0067 & 0.58 & 0.59 & 0.58 \\
\hline & & $\mathrm{AE}$ & 0.55 & {$[0.54-0.57]$} & 0.008 & 0.57 & 0.58 & 0.57 \\
\hline & & LASSO & 0.76 & {$[0.74-0.77]$} & 0.0067 & 0.7 & 0.73 & 0.69 \\
\hline & & LDA & 0.69 & {$[0.67-0.7]$} & 0.0073 & 0.65 & 0.66 & 0.65 \\
\hline & & LR & 0.76 & {$[0.75-0.77]$} & 0.0067 & 0.7 & 0.73 & 0.69 \\
\hline & & NB & 0.77 & {$[0.76-0.78]$} & 0.0062 & 0.73 & 0.7 & 0.73 \\
\hline & & $\mathrm{RF}$ & 0.64 & {$[0.62-0.65]$} & 0.0071 & 0.54 & 0.66 & 0.53 \\
\hline & \multirow{7}{*}{ KW } & $\mathrm{AB}$ & 0.53 & {$[0.52-0.55]$} & 0.0077 & 0.51 & 0.56 & 0.5 \\
\hline & & $\mathrm{AE}$ & 0.61 & {$[0.59-0.62]$} & 0.0075 & 0.52 & 0.66 & 0.51 \\
\hline & & LASSO & 0.71 & {$[0.7-0.73]$} & 0.0069 & 0.65 & 0.71 & 0.64 \\
\hline & & LDA & 0.56 & {$[0.54-0.57]$} & 0.0078 & 0.52 & 0.59 & 0.51 \\
\hline & & LR & 0.71 & {$[0.69-0.72]$} & 0.0071 & 0.65 & 0.68 & 0.64 \\
\hline & & NB & 0.75 & {$[0.73-0.76]$} & 0.0067 & 0.7 & 0.72 & 0.69 \\
\hline & & $\mathrm{RF}$ & 0.67 & {$[0.66-0.68]$} & 0.0071 & 0.54 & 0.73 & 0.51 \\
\hline & \multirow{5}{*}{ Relief } & $\mathrm{AB}$ & 0.68 & {$[0.67-0.69]$} & 0.0066 & 0.58 & 0.71 & 0.56 \\
\hline & & $\mathrm{AE}$ & 0.56 & [0.54-0.57] & 0.0064 & 0.44 & 0.78 & 0.4 \\
\hline & & LASSO & 0.72 & {$[0.71-0.73]$} & 0.0066 & 0.65 & 0.71 & 0.64 \\
\hline & & LDA & 0.72 & {$[0.71-0.73]$} & 0.0068 & 0.64 & 0.68 & 0.63 \\
\hline & & LR & 0.73 & {$[0.72-0.74]$} & 0.0066 & 0.66 & 0.71 & 0.65 \\
\hline
\end{tabular}




\begin{tabular}{|c|c|c|c|c|c|c|c|c|}
\hline & & NB & 0.73 & {$[0.72-0.74]$} & 0.0065 & 0.67 & 0.68 & 0.67 \\
\hline & & $\mathrm{RF}$ & 0.67 & {$[0.66-0.68]$} & 0.0068 & 0.62 & 0.64 & 0.61 \\
\hline & \multirow{7}{*}{ RFE } & $\mathrm{AB}$ & 0.61 & [0.59-0.62] & 0.0071 & 0.42 & 0.83 & 0.37 \\
\hline & & $\mathrm{AE}$ & 0.64 & {$[0.62-0.65]$} & 0.0073 & 0.53 & 0.7 & 0.51 \\
\hline & & LASSO & 0.72 & [0.7-0.73] & 0.0066 & 0.66 & 0.66 & 0.66 \\
\hline & & LDA & 0.67 & [0.66-0.69] & 0.0068 & 0.61 & 0.66 & 0.61 \\
\hline & & LR & 0.71 & {$[0.7-0.72]$} & 0.0067 & 0.66 & 0.66 & 0.66 \\
\hline & & NB & 0.7 & [0.68-0.71] & 0.0063 & 0.66 & 0.68 & 0.66 \\
\hline & & $\mathrm{RF}$ & 0.68 & {$[0.66-0.69]$} & 0.0075 & 0.64 & 0.65 & 0.64 \\
\hline \multirow{28}{*}{ Center_10 } & \multirow{7}{*}{ ANOVA } & $\mathrm{AB}$ & 0.7 & {$[0.68-0.71]$} & 0.0073 & 0.61 & 0.67 & 0.6 \\
\hline & & $\mathrm{AE}$ & 0.75 & {$[0.73-0.76]$} & 0.0069 & 0.69 & 0.69 & 0.69 \\
\hline & & LASSO & 0.77 & [0.76-0.79] & 0.0062 & 0.72 & 0.7 & 0.73 \\
\hline & & LDA & 0.78 & {$[0.77-0.8]$} & 0.0062 & 0.74 & 0.7 & 0.75 \\
\hline & & LR & 0.75 & {$[0.74-0.77]$} & 0.0063 & 0.7 & 0.71 & 0.7 \\
\hline & & NB & 0.75 & {$[0.74-0.76]$} & 0.0065 & 0.68 & 0.74 & 0.67 \\
\hline & & $\mathrm{RF}$ & 0.67 & {$[0.65-0.68]$} & 0.0074 & 0.61 & 0.66 & 0.61 \\
\hline & & $\mathrm{AB}$ & 0.76 & {$[0.75-0.78]$} & 0.0062 & 0.64 & 0.77 & 0.62 \\
\hline & & $\mathrm{AE}$ & 0.75 & [0.74-0.76] & 0.0066 & 0.64 & 0.75 & 0.63 \\
\hline & & LASSO & 0.78 & {$[0.77-0.8]$} & 0.0063 & 0.7 & 0.74 & 0.7 \\
\hline & KW & LDA & 0.75 & {$[0.74-0.77]$} & 0.0066 & 0.69 & 0.69 & 0.69 \\
\hline & & LR & 0.76 & {$[0.75-0.78]$} & 0.0067 & 0.69 & 0.71 & 0.69 \\
\hline & & NB & 0.75 & [0.74-0.77] & 0.0064 & 0.7 & 0.72 & 0.7 \\
\hline & & RF & 0.7 & [0.68-0.71] & 0.0077 & 0.61 & 0.68 & 0.6 \\
\hline & & $\mathrm{AB}$ & 0.61 & [0.59-0.62] & 0.0078 & 0.58 & 0.58 & 0.58 \\
\hline & & $\mathrm{AE}$ & 0.72 & {$[0.7-0.73]$} & 0.0071 & 0.66 & 0.69 & 0.65 \\
\hline & & LASSO & 0.72 & {$[0.71-0.74]$} & 0.007 & 0.67 & 0.71 & 0.67 \\
\hline & Relief & LDA & 0.7 & [0.69-0.71] & 0.0067 & 0.64 & 0.72 & 0.62 \\
\hline & & LR & 0.73 & {$[0.71-0.74]$} & 0.0071 & 0.69 & 0.69 & 0.69 \\
\hline & & NB & 0.69 & {$[0.68-0.7]$} & 0.0066 & 0.62 & 0.74 & 0.61 \\
\hline & & $\mathrm{RF}$ & 0.66 & {$[0.64-0.67]$} & 0.0073 & 0.6 & 0.65 & 0.59 \\
\hline & & $\mathrm{AB}$ & 0.68 & [0.67-0.7] & 0.0082 & 0.6 & 0.68 & 0.59 \\
\hline & & $\mathrm{AE}$ & 0.73 & {$[0.71-0.74]$} & 0.0071 & 0.64 & 0.71 & 0.63 \\
\hline & & LASSO & 0.7 & [0.68-0.71] & 0.0074 & 0.65 & 0.67 & 0.65 \\
\hline & RFE & LDA & 0.68 & {$[0.67-0.69]$} & 0.0068 & 0.61 & 0.72 & 0.6 \\
\hline & & LR & 0.72 & [0.7-0.73] & 0.0071 & 0.67 & 0.69 & 0.67 \\
\hline & & NB & 0.69 & [0.68-0.71] & 0.0069 & 0.65 & 0.7 & 0.64 \\
\hline & & $\mathrm{RF}$ & 0.67 & [0.66-0.69] & 0.0074 & 0.6 & 0.67 & 0.59 \\
\hline & & $\mathrm{AB}$ & 0.64 & [0.63-0.66] & 0.0068 & 0.54 & 0.76 & 0.52 \\
\hline Center 11 & ANOVA & $\mathrm{AE}$ & 0.76 & {$[0.75-0.78]$} & 0.0067 & 0.7 & 0.71 & 0.7 \\
\hline Center_i & RIVU & LASSO & 0.73 & {$[0.71-0.74]$} & 0.0067 & 0.67 & 0.69 & 0.67 \\
\hline & & LDA & 0.67 & {$[0.65-0.68]$} & 0.0074 & 0.61 & 0.67 & 0.6 \\
\hline
\end{tabular}




\begin{tabular}{|c|c|c|c|c|c|c|c|c|}
\hline & & LR & 0.71 & {$[0.7-0.72]$} & 0.0067 & 0.66 & 0.69 & 0.66 \\
\hline & & NB & 0.74 & {$[0.73-0.75]$} & 0.0066 & 0.68 & 0.73 & 0.67 \\
\hline & & $\mathrm{RF}$ & 0.7 & {$[0.68-0.71]$} & 0.0069 & 0.62 & 0.71 & 0.61 \\
\hline & & $\mathrm{AB}$ & 0.61 & [0.6-0.63] & 0.0075 & 0.53 & 0.72 & 0.51 \\
\hline & & $\mathrm{AE}$ & 0.77 & {$[0.76-0.78]$} & 0.0065 & 0.69 & 0.74 & 0.69 \\
\hline & & LASSO & 0.75 & [0.74-0.76] & 0.0068 & 0.7 & 0.7 & 0.69 \\
\hline & KW & LDA & 0.72 & {$[0.7-0.73]$} & 0.007 & 0.64 & 0.7 & 0.64 \\
\hline & & LR & 0.74 & {$[0.72-0.75]$} & 0.0069 & 0.68 & 0.7 & 0.67 \\
\hline & & NB & 0.74 & {$[0.72-0.75]$} & 0.0067 & 0.7 & 0.7 & 0.7 \\
\hline & & $\mathrm{RF}$ & 0.72 & {$[0.71-0.74]$} & 0.0067 & 0.62 & 0.74 & 0.61 \\
\hline & & $\mathrm{AB}$ & 0.66 & {$[0.65-0.68]$} & 0.0077 & 0.55 & 0.7 & 0.53 \\
\hline & & $\mathrm{AE}$ & 0.69 & {$[0.68-0.71]$} & 0.0069 & 0.59 & 0.73 & 0.57 \\
\hline & & LASSO & 0.77 & {$[0.76-0.78]$} & 0.0062 & 0.64 & 0.79 & 0.62 \\
\hline & Relief & LDA & 0.76 & {$[0.74-0.77]$} & 0.0065 & 0.66 & 0.75 & 0.65 \\
\hline & & LR & 0.77 & {$[0.76-0.78]$} & 0.0066 & 0.69 & 0.73 & 0.69 \\
\hline & & NB & 0.7 & {$[0.69-0.71]$} & 0.0064 & 0.62 & 0.73 & 0.61 \\
\hline & & $\mathrm{RF}$ & 0.7 & {$[0.68-0.71]$} & 0.0072 & 0.64 & 0.66 & 0.64 \\
\hline & & $\mathrm{AB}$ & 0.69 & {$[0.68-0.71]$} & 0.0073 & 0.65 & 0.66 & 0.65 \\
\hline & & $\mathrm{AE}$ & 0.76 & {$[0.75-0.77]$} & 0.0067 & 0.7 & 0.71 & 0.7 \\
\hline & & LASSO & 0.69 & {$[0.68-0.71]$} & 0.0066 & 0.56 & 0.78 & 0.53 \\
\hline & RFE & LDA & 0.66 & [0.64-0.67] & 0.0076 & 0.59 & 0.65 & 0.58 \\
\hline & & LR & 0.68 & [0.66-0.69] & 0.0067 & 0.55 & 0.75 & 0.53 \\
\hline & & NB & 0.64 & {$[0.63-0.65]$} & 0.0058 & 0.53 & 0.75 & 0.5 \\
\hline & & $\mathrm{RF}$ & 0.72 & {$[0.7-0.73]$} & 0.0071 & 0.66 & 0.69 & 0.65 \\
\hline & & $\mathrm{AB}$ & 0.73 & {$[0.71-0.74]$} & 0.0074 & 0.68 & 0.68 & 0.68 \\
\hline & & $\mathrm{AE}$ & 0.75 & {$[0.74-0.76]$} & 0.0066 & 0.69 & 0.68 & 0.69 \\
\hline & & LASSO & 0.74 & {$[0.73-0.75]$} & 0.0066 & 0.68 & 0.69 & 0.68 \\
\hline & ANOVA & LDA & 0.67 & {$[0.66-0.68]$} & 0.007 & 0.65 & 0.67 & 0.65 \\
\hline & & LR & 0.75 & [0.74-0.77] & 0.0071 & 0.72 & 0.67 & 0.72 \\
\hline & & NB & 0.73 & {$[0.71-0.74]$} & 0.0068 & 0.68 & 0.7 & 0.67 \\
\hline & & $\mathrm{RF}$ & 0.65 & {$[0.63-0.66]$} & 0.0082 & 0.59 & 0.62 & 0.58 \\
\hline & & $\mathrm{AB}$ & 0.71 & {$[0.7-0.73]$} & 0.0071 & 0.59 & 0.73 & 0.57 \\
\hline Center_12 & & $\mathrm{AE}$ & 0.74 & {$[0.73-0.76]$} & 0.0066 & 0.67 & 0.73 & 0.66 \\
\hline & & LASSO & 0.69 & {$[0.68-0.7]$} & 0.0071 & 0.66 & 0.67 & 0.65 \\
\hline & KW & LDA & 0.61 & {$[0.59-0.62]$} & 0.0073 & 0.55 & 0.67 & 0.53 \\
\hline & & LR & 0.69 & {$[0.68-0.71]$} & 0.0071 & 0.66 & 0.67 & 0.66 \\
\hline & & NB & 0.79 & {$[0.77-0.8]$} & 0.0063 & 0.74 & 0.7 & 0.75 \\
\hline & & $\mathrm{RF}$ & 0.64 & {$[0.62-0.66]$} & 0.0081 & 0.56 & 0.63 & 0.55 \\
\hline & & $\mathrm{AB}$ & 0.69 & {$[0.68-0.71]$} & 0.0077 & 0.59 & 0.71 & 0.58 \\
\hline & Relief & $\mathrm{AE}$ & 0.7 & {$[0.69-0.72]$} & 0.0075 & 0.65 & 0.68 & 0.64 \\
\hline & & LASSO & 0.71 & {$[0.69-0.72]$} & 0.0068 & 0.63 & 0.72 & 0.62 \\
\hline
\end{tabular}




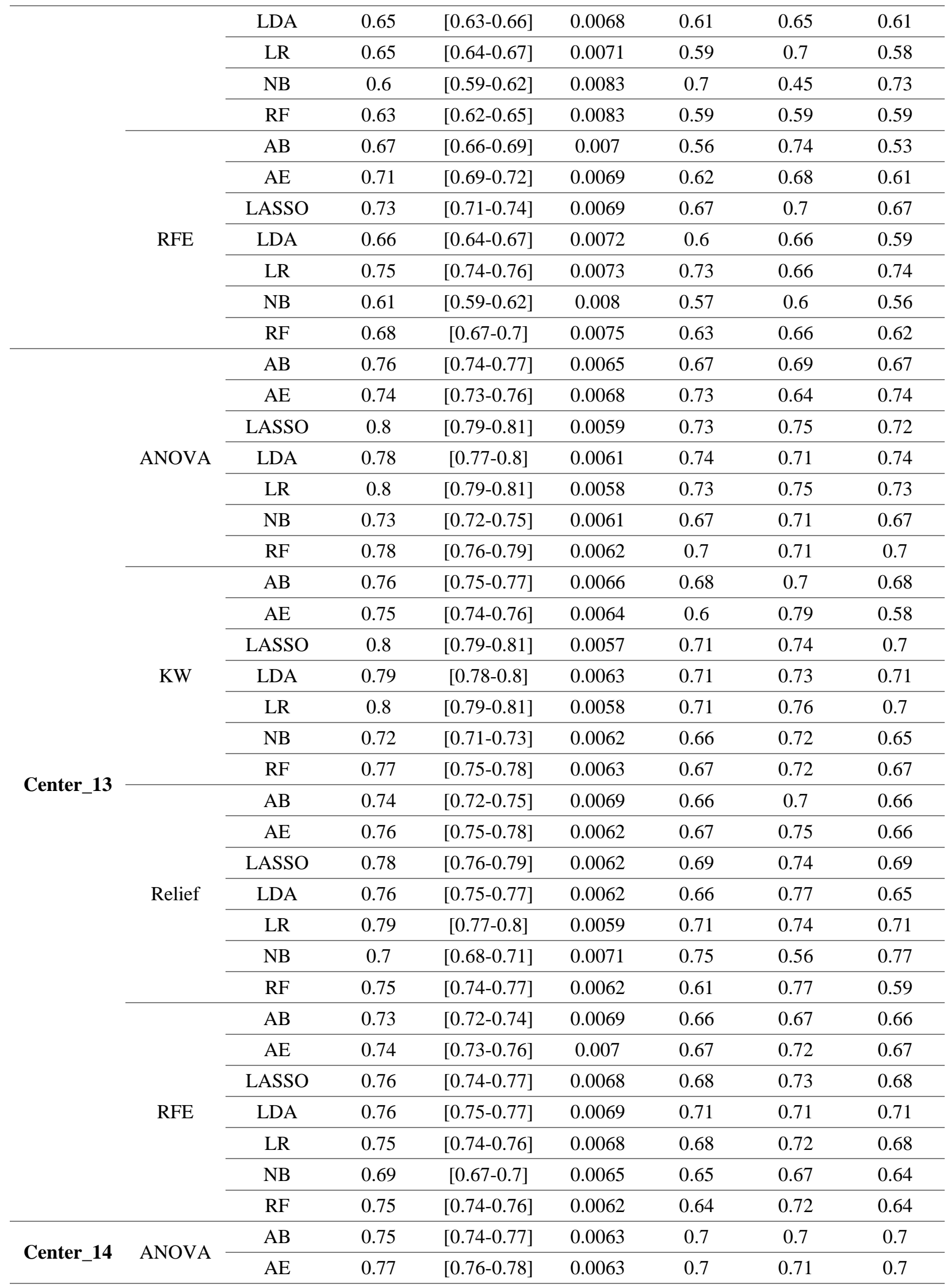




\begin{tabular}{|c|c|c|c|c|c|c|c|c|}
\hline & & LASSO & 0.78 & {$[0.77-0.79]$} & 0.006 & 0.71 & 0.74 & 0.71 \\
\hline & & LDA & 0.74 & {$[0.73-0.75]$} & 0.0063 & 0.66 & 0.7 & 0.65 \\
\hline & & LR & 0.78 & {$[0.77-0.79]$} & 0.006 & 0.71 & 0.74 & 0.71 \\
\hline & & NB & 0.75 & {$[0.74-0.77]$} & 0.0063 & 0.71 & 0.71 & 0.71 \\
\hline & & $\mathrm{RF}$ & 0.69 & {$[0.68-0.71]$} & 0.0073 & 0.63 & 0.64 & 0.63 \\
\hline & & $\mathrm{AB}$ & 0.75 & [0.74-0.76] & 0.0065 & 0.68 & 0.71 & 0.68 \\
\hline & & $\mathrm{AE}$ & 0.77 & {$[0.76-0.78]$} & 0.0062 & 0.7 & 0.71 & 0.7 \\
\hline & & LASSO & 0.8 & {$[0.79-0.81]$} & 0.0057 & 0.72 & 0.75 & 0.72 \\
\hline & KW & LDA & 0.78 & {$[0.77-0.79]$} & 0.0061 & 0.71 & 0.72 & 0.7 \\
\hline & & LR & 0.8 & {$[0.79-0.81]$} & 0.0056 & 0.72 & 0.74 & 0.72 \\
\hline & & NB & 0.79 & {$[0.78-0.81]$} & 0.0059 & 0.72 & 0.75 & 0.71 \\
\hline & & $\mathrm{RF}$ & 0.73 & {$[0.72-0.75]$} & 0.0064 & 0.68 & 0.69 & 0.68 \\
\hline & & $\mathrm{AB}$ & 0.73 & {$[0.72-0.74]$} & 0.0064 & 0.62 & 0.75 & 0.61 \\
\hline & & $\mathrm{AE}$ & 0.77 & {$[0.76-0.78]$} & 0.006 & 0.7 & 0.73 & 0.69 \\
\hline & & LASSO & 0.78 & {$[0.77-0.79]$} & 0.0059 & 0.71 & 0.73 & 0.71 \\
\hline & Relief & LDA & 0.74 & {$[0.73-0.76]$} & 0.0062 & 0.67 & 0.73 & 0.67 \\
\hline & & LR & 0.79 & {$[0.78-0.8]$} & 0.0059 & 0.71 & 0.74 & 0.7 \\
\hline & & NB & 0.64 & {$[0.62-0.65]$} & 0.0075 & 0.67 & 0.58 & 0.68 \\
\hline & & $\mathrm{RF}$ & 0.69 & {$[0.67-0.7]$} & 0.0072 & 0.59 & 0.68 & 0.58 \\
\hline & & $\mathrm{AB}$ & 0.72 & {$[0.71-0.73]$} & 0.0066 & 0.63 & 0.71 & 0.62 \\
\hline & & $\mathrm{AE}$ & 0.78 & {$[0.77-0.79]$} & 0.0058 & 0.7 & 0.73 & 0.7 \\
\hline & & LASSO & 0.77 & {$[0.76-0.78]$} & 0.006 & 0.67 & 0.75 & 0.66 \\
\hline & RFE & LDA & 0.78 & {$[0.77-0.79]$} & 0.006 & 0.68 & 0.74 & 0.68 \\
\hline & & LR & 0.78 & {$[0.76-0.79]$} & 0.006 & 0.68 & 0.74 & 0.67 \\
\hline & & NB & 0.71 & {$[0.69-0.72]$} & 0.0064 & 0.64 & 0.71 & 0.64 \\
\hline & & $\mathrm{RF}$ & 0.68 & {$[0.67-0.69]$} & 0.0071 & 0.59 & 0.68 & 0.57 \\
\hline & & $\mathrm{AB}$ & 0.55 & [0.54-0.57] & 0.0074 & 0.55 & 0.57 & 0.55 \\
\hline & & $\mathrm{AE}$ & 0.61 & {$[0.6-0.63]$} & 0.007 & 0.57 & 0.6 & 0.56 \\
\hline & & LASSO & 0.67 & [0.66-0.68] & 0.0067 & 0.6 & 0.67 & 0.59 \\
\hline & ANOVA & LDA & 0.6 & {$[0.58-0.61]$} & 0.0072 & 0.53 & 0.67 & 0.51 \\
\hline & & LR & 0.66 & {$[0.64-0.67]$} & 0.0069 & 0.57 & 0.69 & 0.56 \\
\hline & & NB & 0.71 & {$[0.7-0.72]$} & 0.0059 & 0.63 & 0.74 & 0.61 \\
\hline & & $\mathrm{RF}$ & 0.56 & [0.54-0.57] & 0.0078 & 0.57 & 0.52 & 0.57 \\
\hline Center_15 & & $\mathrm{AB}$ & 0.55 & [0.54-0.57] & 0.0074 & 0.54 & 0.59 & 0.54 \\
\hline & & $\mathrm{AE}$ & 0.56 & {$[0.55-0.57]$} & 0.0069 & 0.3 & 0.92 & 0.22 \\
\hline & & LASSO & 0.66 & {$[0.64-0.67]$} & 0.0078 & 0.59 & 0.67 & 0.58 \\
\hline & KW & LDA & 0.7 & {$[0.69-0.72]$} & 0.0071 & 0.64 & 0.68 & 0.63 \\
\hline & & LR & 0.68 & [0.66-0.69] & 0.0075 & 0.61 & 0.69 & 0.6 \\
\hline & & NB & 0.54 & {$[0.52-0.56]$} & 0.0081 & 0.53 & 0.56 & 0.52 \\
\hline & & $\mathrm{RF}$ & 0.54 & {$[0.52-0.55]$} & 0.0076 & 0.54 & 0.54 & 0.54 \\
\hline & Relief & $\mathrm{AB}$ & 0.62 & {$[0.6-0.63]$} & 0.0068 & 0.53 & 0.71 & 0.5 \\
\hline
\end{tabular}




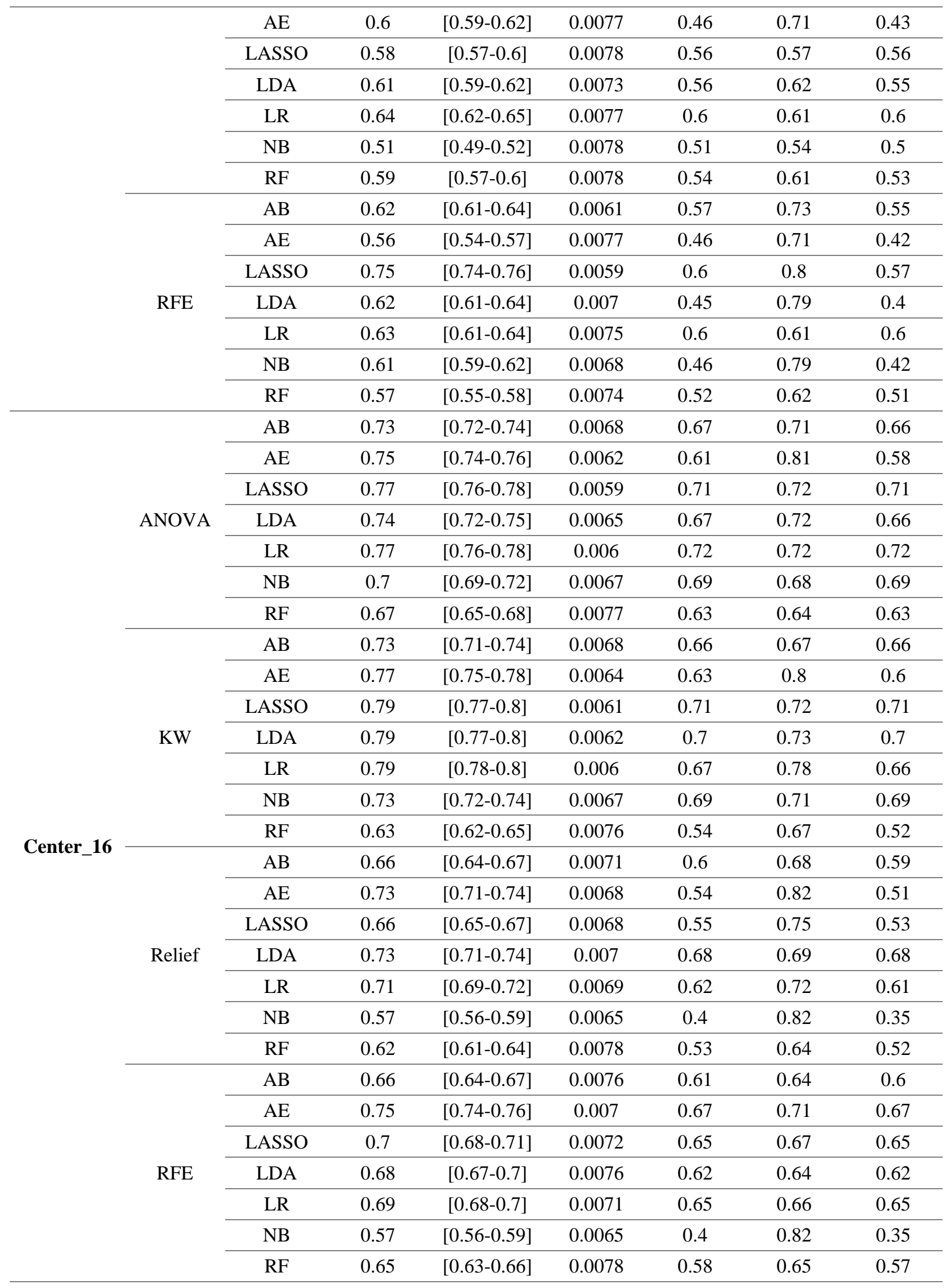




\begin{tabular}{|c|c|c|c|c|c|c|c|c|}
\hline \multirow{28}{*}{ Center_17 } & \multirow{7}{*}{ ANOVA } & $\mathrm{AB}$ & 0.65 & {$[0.64-0.67]$} & 0.0073 & 0.62 & 0.6 & 0.62 \\
\hline & & $\mathrm{AE}$ & 0.76 & {$[0.74-0.77]$} & 0.0064 & 0.66 & 0.72 & 0.65 \\
\hline & & LASSO & 0.73 & [0.71-0.74] & 0.0062 & 0.67 & 0.73 & 0.66 \\
\hline & & LDA & 0.71 & {$[0.69-0.72]$} & 0.0064 & 0.65 & 0.7 & 0.65 \\
\hline & & LR & 0.72 & {$[0.7-0.73]$} & 0.0064 & 0.67 & 0.71 & 0.66 \\
\hline & & NB & 0.69 & {$[0.68-0.71]$} & 0.0069 & 0.67 & 0.68 & 0.67 \\
\hline & & $\mathrm{RF}$ & 0.56 & {$[0.55-0.58]$} & 0.0076 & 0.53 & 0.6 & 0.53 \\
\hline & \multirow{7}{*}{ KW } & $\mathrm{AB}$ & 0.55 & {$[0.54-0.57]$} & 0.0079 & 0.52 & 0.56 & 0.52 \\
\hline & & $\mathrm{AE}$ & 0.78 & {$[0.77-0.79]$} & 0.0063 & 0.67 & 0.77 & 0.66 \\
\hline & & LASSO & 0.71 & {$[0.69-0.72]$} & 0.0064 & 0.63 & 0.72 & 0.62 \\
\hline & & LDA & 0.68 & {$[0.67-0.69]$} & 0.0065 & 0.64 & 0.67 & 0.63 \\
\hline & & LR & 0.7 & {$[0.69-0.71]$} & 0.0063 & 0.63 & 0.71 & 0.62 \\
\hline & & NB & 0.69 & {$[0.68-0.71]$} & 0.0067 & 0.68 & 0.68 & 0.68 \\
\hline & & $\mathrm{RF}$ & 0.54 & {$[0.53-0.56]$} & 0.0072 & 0.56 & 0.53 & 0.57 \\
\hline & \multirow{7}{*}{ Relief } & $\mathrm{AB}$ & 0.58 & [0.56-0.59] & 0.008 & 0.53 & 0.58 & 0.52 \\
\hline & & $\mathrm{AE}$ & 0.74 & {$[0.72-0.75]$} & 0.007 & 0.66 & 0.71 & 0.66 \\
\hline & & LASSO & 0.64 & {$[0.63-0.66]$} & 0.0079 & 0.59 & 0.65 & 0.58 \\
\hline & & LDA & 0.63 & {$[0.62-0.65]$} & 0.0079 & 0.59 & 0.65 & 0.58 \\
\hline & & LR & 0.69 & [0.67-0.7] & 0.0073 & 0.6 & 0.72 & 0.59 \\
\hline & & NB & 0.56 & {$[0.55-0.58]$} & 0.0078 & 0.54 & 0.62 & 0.54 \\
\hline & & $\mathrm{RF}$ & 0.6 & {$[0.58-0.61]$} & 0.008 & 0.41 & 0.77 & 0.37 \\
\hline & \multirow{7}{*}{ RFE } & $\mathrm{AB}$ & 0.56 & {$[0.55-0.58]$} & 0.0079 & 0.54 & 0.6 & 0.53 \\
\hline & & $\mathrm{AE}$ & 0.76 & {$[0.74-0.77]$} & 0.0065 & 0.67 & 0.74 & 0.66 \\
\hline & & LASSO & 0.7 & [0.68-0.71] & 0.0065 & 0.66 & 0.7 & 0.66 \\
\hline & & LDA & 0.68 & [0.66-0.69] & 0.0067 & 0.64 & 0.7 & 0.63 \\
\hline & & LR & 0.72 & {$[0.71-0.73]$} & 0.0063 & 0.66 & 0.73 & 0.65 \\
\hline & & NB & 0.57 & {$[0.55-0.58]$} & 0.0067 & 0.39 & 0.82 & 0.34 \\
\hline & & $\mathrm{RF}$ & 0.58 & [0.56-0.59] & 0.0074 & 0.57 & 0.58 & 0.57 \\
\hline \multirow{13}{*}{ Center_18 } & \multirow{7}{*}{ ANOVA } & $\mathrm{AB}$ & 0.74 & {$[0.73-0.75]$} & 0.0069 & 0.76 & 0.62 & 0.78 \\
\hline & & $\mathrm{AE}$ & 0.71 & [0.69-0.72] & 0.0067 & 0.64 & 0.69 & 0.63 \\
\hline & & LASSO & 0.77 & {$[0.76-0.79]$} & 0.0062 & 0.73 & 0.7 & 0.74 \\
\hline & & LDA & 0.75 & {$[0.74-0.77]$} & 0.0065 & 0.67 & 0.73 & 0.67 \\
\hline & & LR & 0.77 & [0.76-0.78] & 0.0062 & 0.74 & 0.68 & 0.75 \\
\hline & & NB & 0.77 & {$[0.76-0.79]$} & 0.0061 & 0.71 & 0.73 & 0.71 \\
\hline & & $\mathrm{RF}$ & 0.76 & [0.74-0.77] & 0.0065 & 0.7 & 0.71 & 0.69 \\
\hline & \multirow{6}{*}{ KW } & $\mathrm{AB}$ & 0.69 & {$[0.68-0.71]$} & 0.007 & 0.57 & 0.73 & 0.55 \\
\hline & & $\mathrm{AE}$ & 0.71 & {$[0.7-0.73]$} & 0.0067 & 0.66 & 0.66 & 0.66 \\
\hline & & LASSO & 0.75 & {$[0.74-0.77]$} & 0.0064 & 0.69 & 0.71 & 0.69 \\
\hline & & LDA & 0.62 & {$[0.61-0.63]$} & 0.0066 & 0.53 & 0.74 & 0.51 \\
\hline & & LR & 0.58 & [0.56-0.59] & 0.0066 & 0.53 & 0.7 & 0.5 \\
\hline & & NB & 0.76 & {$[0.75-0.77]$} & 0.0058 & 0.71 & 0.72 & 0.71 \\
\hline
\end{tabular}




\begin{tabular}{|c|c|c|c|c|c|c|c|c|}
\hline & & $\mathrm{RF}$ & 0.69 & {$[0.68-0.71]$} & 0.0072 & 0.6 & 0.69 & 0.59 \\
\hline & & $\mathrm{AB}$ & 0.56 & {$[0.55-0.58]$} & 0.0076 & 0.57 & 0.58 & 0.57 \\
\hline & & $\mathrm{AE}$ & 0.74 & [0.73-0.75] & 0.0066 & 0.68 & 0.68 & 0.68 \\
\hline & & LASSO & 0.58 & [0.56-0.59] & 0.0073 & 0.54 & 0.63 & 0.52 \\
\hline & Relief & LDA & 0.66 & [0.65-0.67] & 0.0073 & 0.61 & 0.64 & 0.6 \\
\hline & & LR & 0.67 & [0.66-0.69] & 0.0067 & 0.62 & 0.64 & 0.62 \\
\hline & & NB & 0.56 & [0.54-0.57] & 0.0075 & 0.56 & 0.61 & 0.55 \\
\hline & & $\mathrm{RF}$ & 0.68 & [0.67-0.69] & 0.0072 & 0.61 & 0.65 & 0.61 \\
\hline & & $\mathrm{AB}$ & 0.7 & {$[0.68-0.71]$} & 0.0072 & 0.62 & 0.68 & 0.61 \\
\hline & & $\mathrm{AE}$ & 0.72 & [0.71-0.74] & 0.0069 & 0.67 & 0.68 & 0.66 \\
\hline & & LASSO & 0.57 & [0.56-0.58] & 0.0067 & 0.53 & 0.68 & 0.51 \\
\hline & RFE & LDA & 0.62 & [0.61-0.63] & 0.0067 & 0.59 & 0.65 & 0.58 \\
\hline & & LR & 0.57 & [0.56-0.58] & 0.0067 & 0.53 & 0.7 & 0.5 \\
\hline & & NB & 0.63 & [0.62-0.64] & 0.0065 & 0.53 & 0.73 & 0.5 \\
\hline & & $\mathrm{RF}$ & 0.69 & {$[0.67-0.7]$} & 0.0069 & 0.62 & 0.68 & 0.61 \\
\hline \multirow{26}{*}{ Center_19 } & \multirow{7}{*}{ ANOVA } & $\mathrm{AB}$ & 0.66 & [0.64-0.67] & 0.0079 & 0.64 & 0.6 & 0.65 \\
\hline & & $\mathrm{AE}$ & 0.67 & [0.66-0.69] & 0.0071 & 0.47 & 0.83 & 0.43 \\
\hline & & LASSO & 0.71 & {$[0.7-0.72]$} & 0.0069 & 0.65 & 0.7 & 0.64 \\
\hline & & LDA & 0.75 & [0.74-0.77] & 0.007 & 0.71 & 0.7 & 0.71 \\
\hline & & LR & 0.73 & [0.71-0.74] & 0.0072 & 0.69 & 0.66 & 0.69 \\
\hline & & NB & 0.72 & [0.7-0.73] & 0.0069 & 0.66 & 0.7 & 0.65 \\
\hline & & $\mathrm{RF}$ & 0.58 & {$[0.57-0.6]$} & 0.0077 & 0.41 & 0.78 & 0.36 \\
\hline & \multirow{7}{*}{$\mathrm{KW}$} & $\mathrm{AB}$ & 0.63 & [0.61-0.64] & 0.0072 & 0.53 & 0.69 & 0.5 \\
\hline & & $\mathrm{AE}$ & 0.63 & [0.62-0.65] & 0.0075 & 0.58 & 0.65 & 0.57 \\
\hline & & LASSO & 0.74 & [0.73-0.76] & 0.0066 & 0.69 & 0.71 & 0.69 \\
\hline & & LDA & 0.74 & [0.73-0.75] & 0.0066 & 0.68 & 0.7 & 0.68 \\
\hline & & LR & 0.61 & {$[0.6-0.62]$} & 0.0069 & 0.58 & 0.67 & 0.57 \\
\hline & & NB & 0.74 & {$[0.73-0.76]$} & 0.0067 & 0.69 & 0.71 & 0.69 \\
\hline & & $\mathrm{RF}$ & 0.55 & [0.54-0.57] & 0.0075 & 0.36 & 0.82 & 0.29 \\
\hline & \multirow{7}{*}{ Relief } & $\mathrm{AB}$ & 0.62 & [0.61-0.64] & 0.0079 & 0.57 & 0.61 & 0.56 \\
\hline & & $\mathrm{AE}$ & 0.73 & [0.71-0.74] & 0.0067 & 0.66 & 0.69 & 0.65 \\
\hline & & LASSO & 0.61 & {$[0.6-0.63]$} & 0.007 & 0.51 & 0.77 & 0.47 \\
\hline & & LDA & 0.66 & [0.64-0.68] & 0.0081 & 0.64 & 0.64 & 0.64 \\
\hline & & LR & 0.58 & [0.56-0.59] & 0.0069 & 0.45 & 0.8 & 0.41 \\
\hline & & NB & 0.51 & [0.49-0.52] & 0.0078 & 0.51 & 0.53 & 0.5 \\
\hline & & $\mathrm{RF}$ & 0.55 & [0.54-0.57] & 0.0071 & 0.53 & 0.59 & 0.53 \\
\hline & \multirow{5}{*}{ RFE } & $\mathrm{AB}$ & 0.66 & [0.64-0.67] & 0.007 & 0.56 & 0.7 & 0.54 \\
\hline & & $\mathrm{AE}$ & 0.65 & [0.64-0.67] & 0.0071 & 0.59 & 0.67 & 0.58 \\
\hline & & LASSO & 0.66 & [0.64-0.67] & 0.007 & 0.54 & 0.75 & 0.51 \\
\hline & & LDA & 0.64 & [0.63-0.66] & 0.0072 & 0.53 & 0.75 & 0.5 \\
\hline & & LR & 0.67 & [0.66-0.68] & 0.0069 & 0.55 & 0.74 & 0.53 \\
\hline
\end{tabular}




\begin{tabular}{cccccccc}
\hline NB & 0.63 & {$[0.62-0.64]$} & 0.0067 & 0.53 & 0.75 & 0.5 \\
\cline { 2 - 7 } & RF & 0.53 & {$[0.51-0.54]$} & 0.0073 & 0.52 & 0.57 & 0.52 \\
\hline
\end{tabular}


Supplemental Table 13. Classification performance indices of different feature selector and classifiers in Strategy 12.

\begin{tabular}{|c|c|c|c|c|c|c|c|c|}
\hline Center & FS & Classifier & AUC & 95\% CIs & Std & Acc & Sen & Spe \\
\hline \multirow{28}{*}{ Center_01 } & \multirow{7}{*}{ ANOVA } & $\mathrm{AB}$ & 0.65 & {$[0.55-0.74]$} & 0.0491 & 0.62 & 0.65 & 0.62 \\
\hline & & $\mathrm{AE}$ & 0.64 & {$[0.54-0.74]$} & 0.051 & 0.61 & 0.65 & 0.59 \\
\hline & & LASSO & 0.64 & {$[0.54-0.74]$} & 0.0523 & 0.61 & 0.62 & 0.61 \\
\hline & & LDA & 0.64 & {$[0.54-0.74]$} & 0.0517 & 0.61 & 0.65 & 0.6 \\
\hline & & LR & 0.64 & {$[0.54-0.74]$} & 0.0523 & 0.61 & 0.62 & 0.61 \\
\hline & & NB & 0.65 & {$[0.55-0.76]$} & 0.0519 & 0.68 & 0.57 & 0.72 \\
\hline & & $\mathrm{RF}$ & 0.64 & {$[0.54-0.74]$} & 0.0509 & 0.66 & 0.65 & 0.66 \\
\hline & \multirow{7}{*}{$\mathrm{KW}$} & $\mathrm{AB}$ & 0.63 & {$[0.53-0.74]$} & 0.0529 & 0.59 & 0.68 & 0.56 \\
\hline & & $\mathrm{AE}$ & 0.63 & {$[0.53-0.74]$} & 0.0542 & 0.61 & 0.65 & 0.6 \\
\hline & & LASSO & 0.64 & {$[0.53-0.74]$} & 0.0516 & 0.57 & 0.72 & 0.51 \\
\hline & & LDA & 0.64 & {$[0.53-0.74]$} & 0.0518 & 0.57 & 0.7 & 0.52 \\
\hline & & LR & 0.62 & {$[0.52-0.72]$} & 0.0533 & 0.59 & 0.62 & 0.58 \\
\hline & & NB & 0.65 & {$[0.55-0.75]$} & 0.0517 & 0.68 & 0.57 & 0.72 \\
\hline & & $\mathrm{RF}$ & 0.6 & {$[0.5-0.71]$} & 0.0543 & 0.57 & 0.65 & 0.54 \\
\hline & \multirow{7}{*}{ Relief } & $\mathrm{AB}$ & 0.64 & {$[0.53-0.74]$} & 0.0533 & 0.58 & 0.75 & 0.52 \\
\hline & & $\mathrm{AE}$ & 0.61 & {$[0.5-0.71]$} & 0.0554 & 0.58 & 0.68 & 0.54 \\
\hline & & LASSO & 0.62 & {$[0.5-0.72]$} & 0.0537 & 0.62 & 0.62 & 0.62 \\
\hline & & LDA & 0.61 & [0.49-0.72] & 0.0555 & 0.61 & 0.62 & 0.61 \\
\hline & & LR & 0.67 & {$[0.57-0.76]$} & 0.0498 & 0.66 & 0.68 & 0.65 \\
\hline & & NB & 0.65 & {$[0.54-0.75]$} & 0.0519 & 0.68 & 0.57 & 0.71 \\
\hline & & $\mathrm{RF}$ & 0.6 & {$[0.49-0.71]$} & 0.0557 & 0.6 & 0.68 & 0.57 \\
\hline & \multirow{7}{*}{ RFE } & $\mathrm{AB}$ & 0.64 & {$[0.54-0.73]$} & 0.0496 & 0.57 & 0.75 & 0.51 \\
\hline & & $\mathrm{AE}$ & 0.63 & {$[0.52-0.73]$} & 0.0527 & 0.57 & 0.7 & 0.53 \\
\hline & & LASSO & 0.65 & {$[0.55-0.76]$} & 0.0531 & 0.76 & 0.35 & 0.9 \\
\hline & & LDA & 0.63 & {$[0.52-0.74]$} & 0.0553 & 0.57 & 0.75 & 0.5 \\
\hline & & LR & 0.63 & {$[0.52-0.74]$} & 0.0535 & 0.62 & 0.65 & 0.61 \\
\hline & & NB & 0.67 & {$[0.56-0.76]$} & 0.0506 & 0.66 & 0.62 & 0.68 \\
\hline & & $\mathrm{RF}$ & 0.61 & {$[0.51-0.72]$} & 0.0541 & 0.59 & 0.7 & 0.54 \\
\hline \multirow{10}{*}{ Center_02 } & \multirow{7}{*}{ ANOVA } & $\mathrm{AB}$ & 0.86 & {$[0.8-0.9]$} & 0.0244 & 0.8 & 0.87 & 0.74 \\
\hline & & $\mathrm{AE}$ & 0.86 & [0.8-0.9] & 0.0252 & 0.8 & 0.81 & 0.8 \\
\hline & & LASSO & 0.86 & {$[0.8-0.9]$} & 0.0253 & 0.81 & 0.82 & 0.8 \\
\hline & & LDA & 0.85 & [0.8-0.9] & 0.0248 & 0.8 & 0.81 & 0.8 \\
\hline & & LR & 0.85 & {$[0.8-0.9]$} & 0.0251 & 0.8 & 0.81 & 0.79 \\
\hline & & NB & 0.84 & {$[0.78-0.88]$} & 0.0258 & 0.78 & 0.68 & 0.86 \\
\hline & & $\mathrm{RF}$ & 0.93 & [0.89-0.96] & 0.018 & 0.85 & 0.9 & 0.81 \\
\hline & \multirow{3}{*}{$\mathrm{KW}$} & $\mathrm{AB}$ & 0.86 & {$[0.8-0.9]$} & 0.0244 & 0.79 & 0.83 & 0.76 \\
\hline & & $\mathrm{AE}$ & 0.86 & [0.8-0.9] & 0.0247 & 0.79 & 0.79 & 0.79 \\
\hline & & LASSO & 0.86 & {$[0.8-0.9]$} & 0.0246 & 0.79 & 0.82 & 0.77 \\
\hline
\end{tabular}




\begin{tabular}{|c|c|c|c|c|c|c|c|c|}
\hline & & LDA & 0.85 & {$[0.79-0.9]$} & 0.0257 & 0.79 & 0.79 & 0.79 \\
\hline & & LR & 0.85 & [0.79-0.9] & 0.0259 & 0.8 & 0.82 & 0.79 \\
\hline & & NB & 0.84 & [0.78-0.89] & 0.0256 & 0.77 & 0.79 & 0.76 \\
\hline & & $\mathrm{RF}$ & 0.93 & {$[0.9-0.96]$} & 0.0162 & 0.85 & 0.9 & 0.82 \\
\hline & & $\mathrm{AB}$ & 0.86 & [0.81-0.9] & 0.0239 & 0.81 & 0.83 & 0.79 \\
\hline & & $\mathrm{AE}$ & 0.85 & [0.8-0.9] & 0.0252 & 0.79 & 0.82 & 0.77 \\
\hline & & LASSO & 0.85 & [0.8-0.9] & 0.0252 & 0.8 & 0.82 & 0.79 \\
\hline & Relief & LDA & 0.84 & [0.79-0.89] & 0.0258 & 0.8 & 0.81 & 0.79 \\
\hline & & LR & 0.84 & [0.78-0.89] & 0.0265 & 0.78 & 0.85 & 0.74 \\
\hline & & NB & 0.82 & [0.76-0.87] & 0.0266 & 0.76 & 0.77 & 0.75 \\
\hline & & RF & 0.93 & [0.89-0.96] & 0.0163 & 0.86 & 0.89 & 0.84 \\
\hline & & $\mathrm{AB}$ & 0.86 & [0.8-0.9] & 0.0248 & 0.8 & 0.81 & 0.8 \\
\hline & & $\mathrm{AE}$ & 0.86 & [0.8-0.9] & 0.0244 & 0.77 & 0.79 & 0.76 \\
\hline & & LASSO & 0.86 & {$[0.8-0.91]$} & 0.0246 & 0.81 & 0.82 & 0.8 \\
\hline & RFE & LDA & 0.86 & {$[0.81-0.9]$} & 0.0247 & 0.81 & 0.83 & 0.81 \\
\hline & & LR & 0.85 & [0.79-0.9] & 0.0254 & 0.78 & 0.82 & 0.76 \\
\hline & & NB & 0.82 & [0.77-0.87] & 0.0261 & 0.74 & 0.79 & 0.71 \\
\hline & & $\mathrm{RF}$ & 0.92 & [0.88-0.96] & 0.0185 & 0.87 & 0.87 & 0.86 \\
\hline & & $\mathrm{AB}$ & 0.97 & [0.95-0.99] & 0.0108 & 0.96 & 1 & 0.96 \\
\hline & & $\mathrm{AE}$ & 0.97 & [0.95-0.99] & 0.0108 & 0.95 & 1 & 0.95 \\
\hline & & LASSO & 0.97 & [0.94-0.99] & 0.0144 & 0.93 & 1 & 0.93 \\
\hline & ANOVA & LDA & 0.96 & [0.93-0.99] & 0.015 & 0.92 & 1 & 0.92 \\
\hline & & LR & 0.96 & [0.92-0.99] & 0.0176 & 0.91 & 1 & 0.9 \\
\hline & & NB & 0.96 & [0.93-0.98] & 0.015 & 0.92 & 1 & 0.91 \\
\hline & & $\mathrm{RF}$ & 0.95 & [0.92-0.97] & 0.0144 & 0.92 & 1 & 0.92 \\
\hline & & $\mathrm{AB}$ & 0.95 & [0.91-0.98] & 0.0195 & 0.89 & 1 & 0.89 \\
\hline & & $\mathrm{AE}$ & 0.96 & [0.93-0.99] & 0.015 & 0.92 & 1 & 0.92 \\
\hline & & LASSO & 0.96 & [0.93-0.99] & 0.0153 & 0.92 & 1 & 0.92 \\
\hline & $\mathrm{KW}$ & LDA & 0.96 & [0.92-0.99] & 0.0161 & 0.93 & 1 & 0.93 \\
\hline Center_03 & & LR & 0.94 & [0.89-0.98] & 0.0236 & 0.87 & 1 & 0.87 \\
\hline & & NB & 0.95 & [0.91-0.99] & 0.0186 & 0.92 & 1 & 0.91 \\
\hline & & $\mathrm{RF}$ & 0.95 & [0.91-0.98] & 0.0164 & 0.91 & 1 & 0.91 \\
\hline & & $\mathrm{AB}$ & 0.95 & [0.9-0.99] & 0.0238 & 0.88 & 1 & 0.88 \\
\hline & & $\mathrm{AE}$ & 0.98 & [0.95-1] & 0.0113 & 0.95 & 1 & 0.95 \\
\hline & & LASSO & 0.95 & [0.91-0.99] & 0.019 & 0.92 & 1 & 0.91 \\
\hline & Relief & LDA & 0.96 & [0.92-0.99] & 0.0167 & 0.91 & 1 & 0.91 \\
\hline & & LR & 0.95 & [0.89-0.99] & 0.0256 & 0.87 & 1 & 0.86 \\
\hline & & NB & 0.73 & [0.31-0.97] & 0.184 & 0.77 & 0.8 & 0.77 \\
\hline & & $\mathrm{RF}$ & 0.95 & [0.89-0.99] & 0.025 & 0.87 & 1 & 0.86 \\
\hline & DEF & $\mathrm{AB}$ & 0.87 & [0.73-0.98] & 0.0609 & 0.71 & 1 & 0.7 \\
\hline & RFE & $\mathrm{AE}$ & 0.95 & [0.91-0.98] & 0.0202 & 0.89 & 1 & 0.89 \\
\hline
\end{tabular}




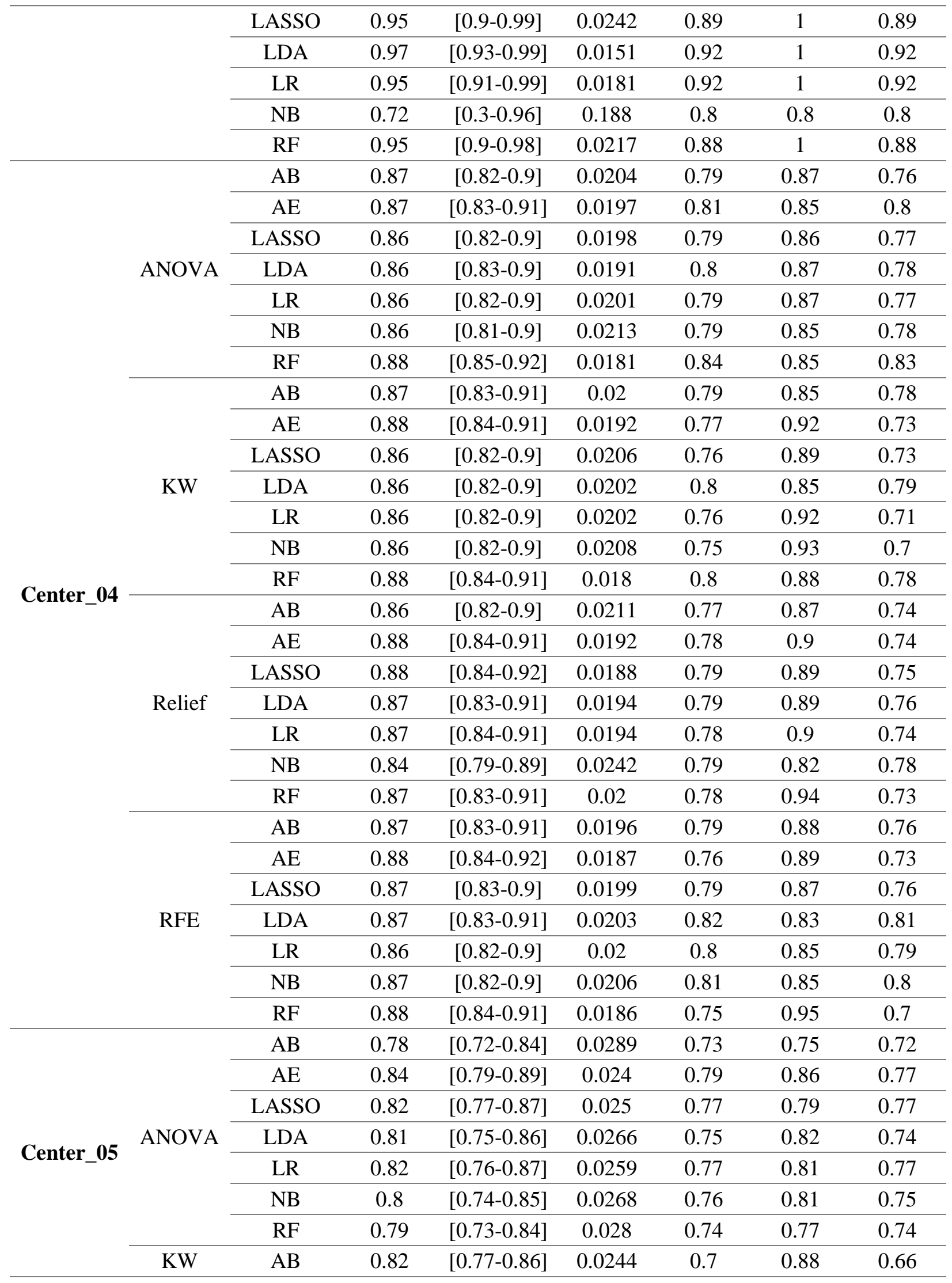




\begin{tabular}{|c|c|c|c|c|c|c|c|c|}
\hline & & $\mathrm{AE}$ & 0.85 & [0.8-0.89] & 0.023 & 0.79 & 0.86 & 0.77 \\
\hline & & LASSO & 0.83 & [0.77-0.87] & 0.0255 & 0.75 & 0.85 & 0.72 \\
\hline & & LDA & 0.81 & [0.76-0.87] & 0.0258 & 0.73 & 0.81 & 0.72 \\
\hline & & LR & 0.82 & [0.77-0.87] & 0.0252 & 0.77 & 0.78 & 0.77 \\
\hline & & NB & 0.81 & [0.76-0.86] & 0.0261 & 0.78 & 0.78 & 0.78 \\
\hline & & $\mathrm{RF}$ & 0.81 & {$[0.75-0.85]$} & 0.0252 & 0.69 & 0.92 & 0.63 \\
\hline & & $\mathrm{AB}$ & 0.79 & [0.73-0.84] & 0.0288 & 0.68 & 0.82 & 0.65 \\
\hline & & $\mathrm{AE}$ & 0.84 & [0.79-0.89] & 0.0232 & 0.76 & 0.88 & 0.73 \\
\hline & & LASSO & 0.82 & [0.77-0.86] & 0.026 & 0.75 & 0.79 & 0.73 \\
\hline & Relief & LDA & 0.8 & [0.74-0.85] & 0.0283 & 0.73 & 0.81 & 0.71 \\
\hline & & LR & 0.79 & [0.74-0.84] & 0.0261 & 0.74 & 0.75 & 0.73 \\
\hline & & NB & 0.8 & [0.74-0.85] & 0.0282 & 0.78 & 0.78 & 0.78 \\
\hline & & $\mathrm{RF}$ & 0.79 & [0.74-0.84] & 0.0276 & 0.72 & 0.77 & 0.71 \\
\hline & & $\mathrm{AB}$ & 0.81 & {$[0.75-0.86]$} & 0.0279 & 0.74 & 0.86 & 0.72 \\
\hline & & $\mathrm{AE}$ & 0.86 & [0.81-0.9] & 0.0229 & 0.78 & 0.89 & 0.76 \\
\hline & & LASSO & 0.83 & [0.78-0.88] & 0.0249 & 0.72 & 0.89 & 0.68 \\
\hline & RFE & LDA & 0.81 & [0.76-0.87] & 0.027 & 0.7 & 0.88 & 0.66 \\
\hline & & LR & 0.81 & [0.76-0.86] & 0.0259 & 0.77 & 0.79 & 0.77 \\
\hline & & NB & 0.81 & [0.76-0.86] & 0.0259 & 0.79 & 0.79 & 0.78 \\
\hline & & $\mathrm{RF}$ & 0.81 & {$[0.76-0.86]$} & 0.0257 & 0.7 & 0.85 & 0.67 \\
\hline & & $\mathrm{AB}$ & 0.79 & [0.74-0.83] & 0.0224 & 0.72 & 0.8 & 0.67 \\
\hline & & $\mathrm{AE}$ & 0.8 & {$[0.75-0.84]$} & 0.0212 & 0.73 & 0.79 & 0.7 \\
\hline & & LASSO & 0.82 & [0.78-0.87] & 0.022 & 0.76 & 0.78 & 0.76 \\
\hline & ANOVA & LDA & 0.81 & [0.77-0.85] & 0.0221 & 0.76 & 0.79 & 0.74 \\
\hline & & LR & 0.82 & [0.78-0.87] & 0.0219 & 0.76 & 0.78 & 0.76 \\
\hline & & NB & 0.81 & [0.77-0.85] & 0.0215 & 0.74 & 0.78 & 0.71 \\
\hline & & $\mathrm{RF}$ & 0.79 & [0.74-0.83] & 0.0226 & 0.72 & 0.73 & 0.71 \\
\hline & & $\mathrm{AB}$ & 0.8 & {$[0.75-0.84]$} & 0.0221 & 0.73 & 0.77 & 0.7 \\
\hline & & $\mathrm{AE}$ & 0.81 & [0.76-0.85] & 0.022 & 0.74 & 0.78 & 0.71 \\
\hline & & LASSO & 0.81 & [0.76-0.85] & 0.0221 & 0.74 & 0.76 & 0.73 \\
\hline Center_06 & $\mathrm{KW}$ & LDA & 0.83 & [0.78-0.87] & 0.0216 & 0.77 & 0.78 & 0.76 \\
\hline & & LR & 0.82 & {$[0.77-0.86]$} & 0.0216 & 0.74 & 0.82 & 0.69 \\
\hline & & NB & 0.81 & {$[0.77-0.85]$} & 0.0216 & 0.73 & 0.84 & 0.65 \\
\hline & & $\mathrm{RF}$ & 0.8 & [0.76-0.84] & 0.0221 & 0.71 & 0.8 & 0.66 \\
\hline & & $\mathrm{AB}$ & 0.76 & [0.72-0.81] & 0.0241 & 0.69 & 0.86 & 0.58 \\
\hline & & $\mathrm{AE}$ & 0.76 & [0.71-0.8] & 0.0253 & 0.71 & 0.75 & 0.69 \\
\hline & & LASSO & 0.73 & {$[0.68-0.78]$} & 0.0255 & 0.68 & 0.76 & 0.63 \\
\hline & Relief & LDA & 0.83 & [0.79-0.87] & 0.0206 & 0.76 & 0.78 & 0.75 \\
\hline & & LR & 0.74 & {$[0.7-0.79]$} & 0.0251 & 0.7 & 0.72 & 0.68 \\
\hline & & NB & 0.62 & [0.56-0.68] & 0.0289 & 0.62 & 0.59 & 0.65 \\
\hline & & $\mathrm{RF}$ & 0.78 & [0.73-0.82] & 0.0233 & 0.72 & 0.77 & 0.69 \\
\hline
\end{tabular}




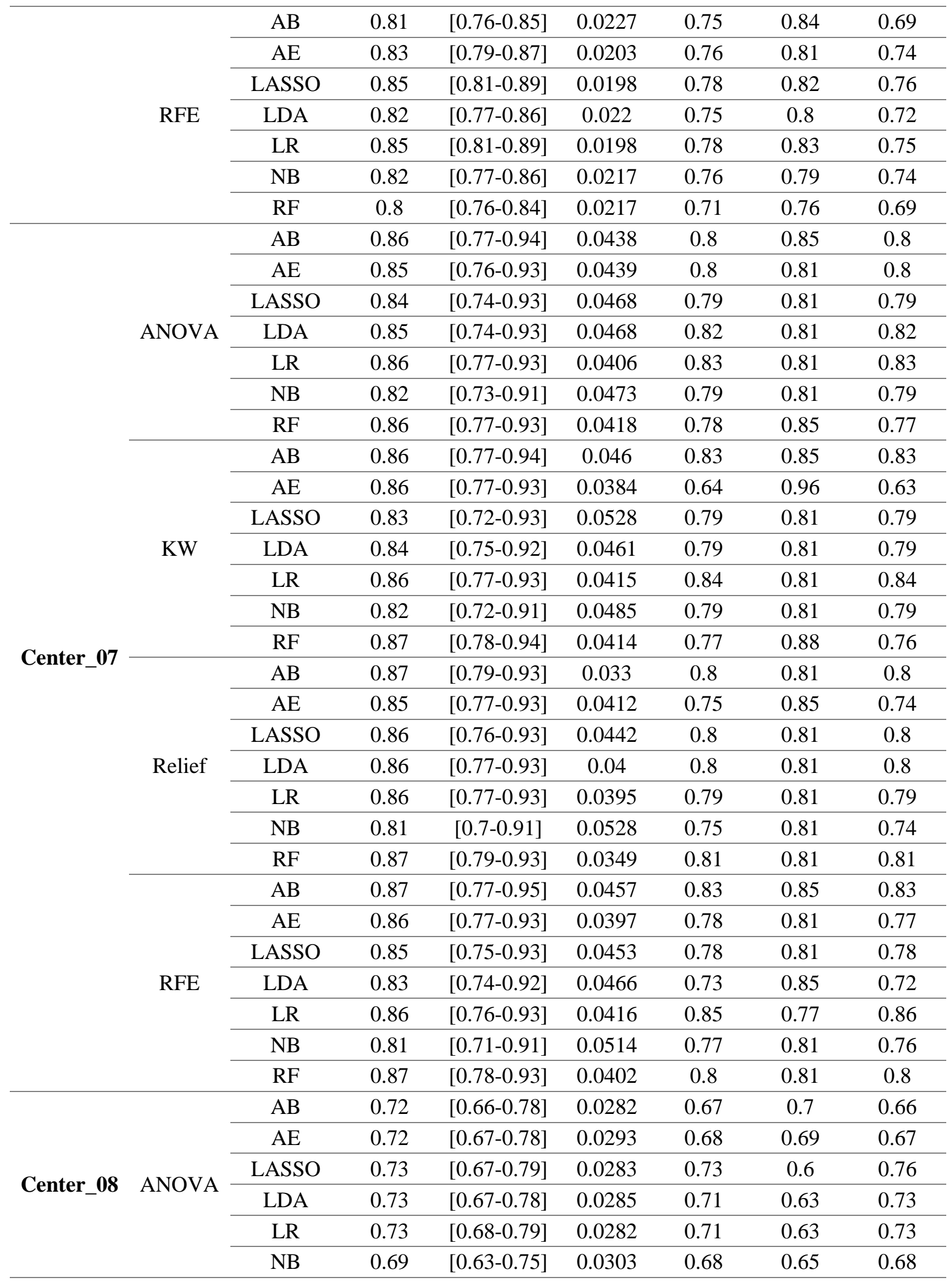




\begin{tabular}{|c|c|c|c|c|c|c|c|c|}
\hline & & RF & 0.73 & {$[0.67-0.78]$} & 0.0287 & 0.68 & 0.73 & 0.66 \\
\hline & \multirow{7}{*}{$\mathrm{KW}$} & $\mathrm{AB}$ & 0.73 & {$[0.67-0.78]$} & 0.0269 & 0.66 & 0.69 & 0.65 \\
\hline & & $\mathrm{AE}$ & 0.74 & [0.68-0.79] & 0.0281 & 0.67 & 0.73 & 0.66 \\
\hline & & LASSO & 0.73 & {$[0.68-0.79]$} & 0.028 & 0.79 & 0.54 & 0.85 \\
\hline & & LDA & 0.74 & [0.68-0.79] & 0.0274 & 0.68 & 0.7 & 0.67 \\
\hline & & LR & 0.72 & {$[0.67-0.78]$} & 0.0288 & 0.79 & 0.5 & 0.86 \\
\hline & & NB & 0.7 & {$[0.64-0.75]$} & 0.0298 & 0.73 & 0.59 & 0.76 \\
\hline & & $\mathrm{RF}$ & 0.72 & [0.67-0.78] & 0.029 & 0.65 & 0.74 & 0.63 \\
\hline & \multirow{7}{*}{ Relief } & $\mathrm{AB}$ & 0.73 & {$[0.67-0.78]$} & 0.0285 & 0.61 & 0.79 & 0.57 \\
\hline & & $\mathrm{AE}$ & 0.73 & [0.68-0.79] & 0.0272 & 0.67 & 0.75 & 0.65 \\
\hline & & LASSO & 0.73 & [0.68-0.79] & 0.028 & 0.68 & 0.72 & 0.67 \\
\hline & & LDA & 0.74 & [0.69-0.79] & 0.0265 & 0.58 & 0.84 & 0.52 \\
\hline & & LR & 0.73 & {$[0.67-0.78]$} & 0.028 & 0.69 & 0.69 & 0.69 \\
\hline & & NB & 0.69 & {$[0.63-0.75]$} & 0.0307 & 0.76 & 0.55 & 0.8 \\
\hline & & $\mathrm{RF}$ & 0.72 & [0.66-0.77] & 0.0275 & 0.62 & 0.75 & 0.59 \\
\hline & \multirow{7}{*}{ RFE } & $\mathrm{AB}$ & 0.73 & {$[0.67-0.78]$} & 0.0282 & 0.69 & 0.69 & 0.69 \\
\hline & & $\mathrm{AE}$ & 0.75 & {$[0.69-0.8]$} & 0.0264 & 0.69 & 0.7 & 0.69 \\
\hline & & LASSO & 0.74 & [0.68-0.79] & 0.0282 & 0.79 & 0.52 & 0.86 \\
\hline & & LDA & 0.71 & {$[0.65-0.77]$} & 0.029 & 0.59 & 0.76 & 0.56 \\
\hline & & LR & 0.72 & [0.66-0.77] & 0.029 & 0.67 & 0.7 & 0.67 \\
\hline & & NB & 0.69 & {$[0.62-0.75]$} & 0.0308 & 0.75 & 0.55 & 0.8 \\
\hline & & $\mathrm{RF}$ & 0.73 & {$[0.67-0.78]$} & 0.0275 & 0.62 & 0.79 & 0.58 \\
\hline \multirow{19}{*}{ Center_09 } & \multirow{7}{*}{ ANOVA } & $\mathrm{AB}$ & 0.75 & [0.6-0.87] & 0.0692 & 0.76 & 0.72 & 0.76 \\
\hline & & $\mathrm{AE}$ & 0.78 & {$[0.63-0.9]$} & 0.0677 & 0.81 & 0.72 & 0.82 \\
\hline & & LASSO & 0.77 & [0.62-0.89] & 0.0702 & 0.74 & 0.78 & 0.74 \\
\hline & & LDA & 0.78 & [0.63-0.9] & 0.0669 & 0.82 & 0.72 & 0.82 \\
\hline & & LR & 0.76 & [0.61-0.88] & 0.0694 & 0.85 & 0.67 & 0.86 \\
\hline & & NB & 0.8 & [0.66-0.9] & 0.0627 & 0.71 & 0.83 & 0.71 \\
\hline & & $\mathrm{RF}$ & 0.72 & [0.57-0.85] & 0.0733 & 0.68 & 0.72 & 0.68 \\
\hline & \multirow{7}{*}{$\mathrm{KW}$} & $\mathrm{AB}$ & 0.77 & [0.66-0.87] & 0.0529 & 0.56 & 0.89 & 0.55 \\
\hline & & $\mathrm{AE}$ & 0.79 & [0.66-0.9] & 0.0602 & 0.81 & 0.67 & 0.81 \\
\hline & & LASSO & 0.78 & {$[0.62-0.91]$} & 0.0728 & 0.7 & 0.83 & 0.7 \\
\hline & & LDA & 0.83 & {$[0.73-0.92]$} & 0.0469 & 0.57 & 0.94 & 0.56 \\
\hline & & LR & 0.75 & {$[0.59-0.88]$} & 0.0745 & 0.87 & 0.67 & 0.87 \\
\hline & & NB & 0.77 & [0.64-0.89] & 0.0618 & 0.7 & 0.83 & 0.7 \\
\hline & & $\mathrm{RF}$ & 0.73 & {$[0.59-0.86]$} & 0.0717 & 0.86 & 0.56 & 0.87 \\
\hline & \multirow{5}{*}{ Relief } & $\mathrm{AB}$ & 0.75 & [0.61-0.87] & 0.0642 & 0.56 & 0.89 & 0.55 \\
\hline & & $\mathrm{AE}$ & 0.79 & [0.64-0.91] & 0.0648 & 0.72 & 0.78 & 0.72 \\
\hline & & LASSO & 0.81 & {$[0.7-0.91]$} & 0.0554 & 0.74 & 0.78 & 0.74 \\
\hline & & LDA & 0.83 & [0.74-0.92] & 0.0455 & 0.69 & 0.83 & 0.69 \\
\hline & & LR & 0.77 & [0.61-0.9] & 0.0715 & 0.74 & 0.83 & 0.74 \\
\hline
\end{tabular}




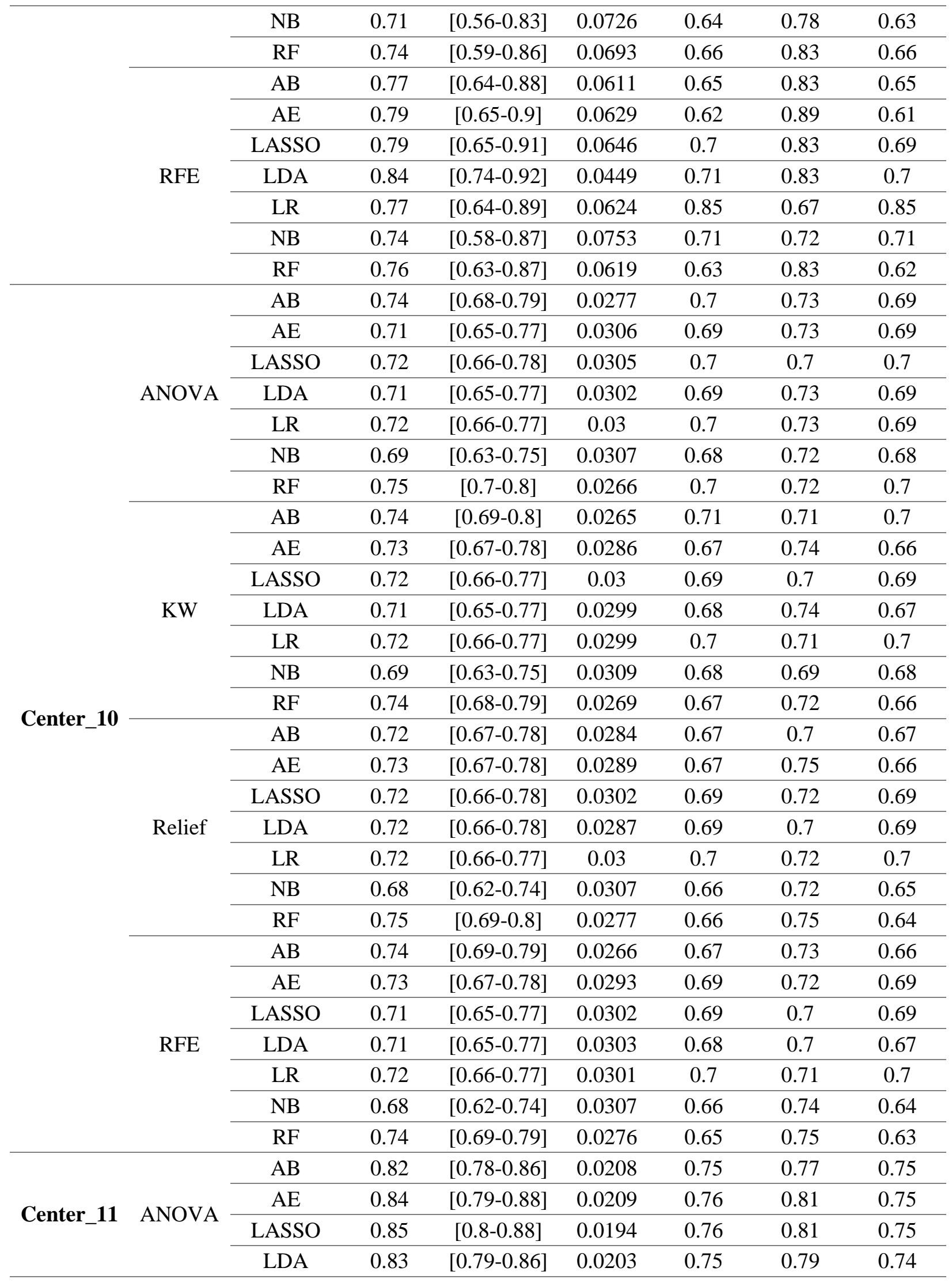




\begin{tabular}{|c|c|c|c|c|c|c|c|c|}
\hline & & LR & 0.83 & [0.79-0.87] & 0.0206 & 0.77 & 0.77 & 0.76 \\
\hline & & NB & 0.81 & {$[0.77-0.85]$} & 0.0198 & 0.73 & 0.79 & 0.72 \\
\hline & & $\mathrm{RF}$ & 0.82 & {$[0.77-0.86]$} & 0.0213 & 0.74 & 0.79 & 0.74 \\
\hline & & $\mathrm{AB}$ & 0.82 & {$[0.78-0.86]$} & 0.0205 & 0.73 & 0.79 & 0.72 \\
\hline & & $\mathrm{AE}$ & 0.84 & {$[0.8-0.87]$} & 0.0196 & 0.75 & 0.83 & 0.74 \\
\hline & & LASSO & 0.85 & {$[0.8-0.88]$} & 0.0193 & 0.74 & 0.83 & 0.73 \\
\hline & KW & LDA & 0.83 & {$[0.78-0.86]$} & 0.0205 & 0.7 & 0.85 & 0.68 \\
\hline & & LR & 0.83 & {$[0.79-0.86]$} & 0.0201 & 0.76 & 0.78 & 0.76 \\
\hline & & NB & 0.81 & {$[0.77-0.85]$} & 0.0199 & 0.73 & 0.79 & 0.72 \\
\hline & & $\mathrm{RF}$ & 0.82 & {$[0.77-0.85]$} & 0.0211 & 0.75 & 0.77 & 0.75 \\
\hline & & $\mathrm{AB}$ & 0.82 & {$[0.77-0.85]$} & 0.0207 & 0.71 & 0.83 & 0.69 \\
\hline & & $\mathrm{AE}$ & 0.84 & {$[0.8-0.88]$} & 0.0202 & 0.77 & 0.81 & 0.76 \\
\hline & & LASSO & 0.85 & [0.81-0.88] & 0.0196 & 0.76 & 0.82 & 0.76 \\
\hline & Relief & LDA & 0.84 & {$[0.8-0.87]$} & 0.0193 & 0.74 & 0.83 & 0.73 \\
\hline & & LR & 0.83 & [0.78-0.87] & 0.0203 & 0.74 & 0.78 & 0.74 \\
\hline & & NB & 0.79 & {$[0.75-0.83]$} & 0.0212 & 0.7 & 0.79 & 0.68 \\
\hline & & $\mathrm{RF}$ & 0.81 & [0.76-0.85] & 0.0224 & 0.7 & 0.85 & 0.68 \\
\hline & & $\mathrm{AB}$ & 0.83 & [0.78-0.86] & 0.0196 & 0.76 & 0.77 & 0.76 \\
\hline & & $\mathrm{AE}$ & 0.84 & {$[0.8-0.88]$} & 0.0201 & 0.74 & 0.85 & 0.73 \\
\hline & & LASSO & 0.84 & {$[0.8-0.88]$} & 0.0199 & 0.75 & 0.82 & 0.74 \\
\hline & RFE & LDA & 0.83 & [0.79-0.87] & 0.0204 & 0.75 & 0.81 & 0.74 \\
\hline & & LR & 0.82 & [0.78-0.86] & 0.0202 & 0.72 & 0.82 & 0.7 \\
\hline & & NB & 0.8 & [0.76-0.84] & 0.0211 & 0.73 & 0.78 & 0.72 \\
\hline & & $\mathrm{RF}$ & 0.82 & {$[0.77-0.86]$} & 0.0211 & 0.71 & 0.83 & 0.69 \\
\hline & & $\mathrm{AB}$ & 0.81 & {$[0.77-0.86]$} & 0.0234 & 0.75 & 0.75 & 0.75 \\
\hline & & $\mathrm{AE}$ & 0.83 & [0.79-0.87] & 0.0216 & 0.78 & 0.78 & 0.78 \\
\hline & & LASSO & 0.82 & {$[0.78-0.86]$} & 0.0222 & 0.72 & 0.82 & 0.7 \\
\hline & ANOVA & LDA & 0.82 & [0.77-0.86] & 0.023 & 0.72 & 0.81 & 0.71 \\
\hline & & LR & 0.82 & [0.78-0.86] & 0.0222 & 0.72 & 0.85 & 0.7 \\
\hline & & NB & 0.81 & [0.76-0.85] & 0.0227 & 0.76 & 0.76 & 0.76 \\
\hline & & $\mathrm{RF}$ & 0.81 & [0.76-0.85] & 0.0229 & 0.73 & 0.77 & 0.73 \\
\hline & & $\mathrm{AB}$ & 0.82 & [0.78-0.87] & 0.023 & 0.76 & 0.77 & 0.75 \\
\hline Center_12 & & $\mathrm{AE}$ & 0.82 & {$[0.78-0.86]$} & 0.0217 & 0.66 & 0.9 & 0.63 \\
\hline & & LASSO & 0.82 & [0.78-0.86] & 0.0227 & 0.7 & 0.83 & 0.68 \\
\hline & KW & LDA & 0.81 & {$[0.77-0.86]$} & 0.0232 & 0.72 & 0.8 & 0.71 \\
\hline & & LR & 0.83 & [0.78-0.87] & 0.0219 & 0.71 & 0.85 & 0.69 \\
\hline & & NB & 0.81 & [0.76-0.85] & 0.0224 & 0.76 & 0.76 & 0.76 \\
\hline & & $\mathrm{RF}$ & 0.81 & {$[0.76-0.85]$} & 0.0227 & 0.72 & 0.78 & 0.71 \\
\hline & & $\mathrm{AB}$ & 0.82 & {$[0.77-0.86]$} & 0.0237 & 0.74 & 0.81 & 0.73 \\
\hline & Relief & $\mathrm{AE}$ & 0.82 & [0.78-0.87] & 0.0219 & 0.71 & 0.86 & 0.69 \\
\hline & & LASSO & 0.82 & [0.78-0.86] & 0.0224 & 0.71 & 0.85 & 0.69 \\
\hline
\end{tabular}




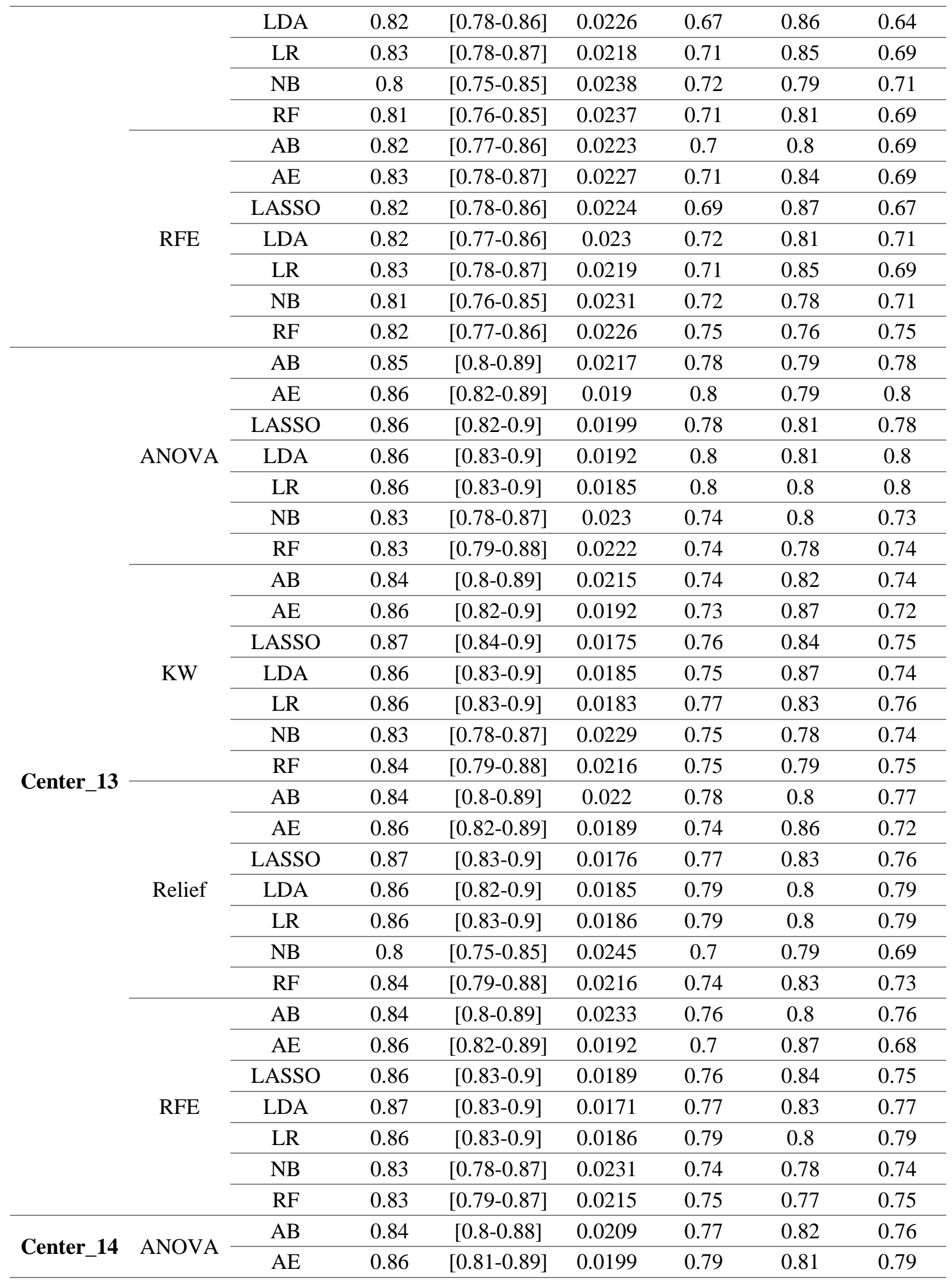




\begin{tabular}{|c|c|c|c|c|c|c|c|c|}
\hline & & LASSO & 0.84 & [0.79-0.89] & 0.0225 & 0.8 & 0.8 & 0.8 \\
\hline & & LDA & 0.85 & [0.8-0.89] & 0.0202 & 0.79 & 0.81 & 0.79 \\
\hline & & LR & 0.85 & [0.81-0.89] & 0.0221 & 0.81 & 0.8 & 0.81 \\
\hline & & NB & 0.82 & {$[0.78-0.87]$} & 0.0243 & 0.81 & 0.76 & 0.81 \\
\hline & & RF & 0.86 & [0.82-0.89] & 0.0174 & 0.79 & 0.8 & 0.79 \\
\hline & \multirow{7}{*}{$\mathrm{KW}$} & $\mathrm{AB}$ & 0.84 & {$[0.8-0.88]$} & 0.0192 & 0.72 & 0.83 & 0.71 \\
\hline & & $\mathrm{AE}$ & 0.85 & [0.81-0.89] & 0.0212 & 0.8 & 0.8 & 0.8 \\
\hline & & LASSO & 0.84 & [0.79-0.88] & 0.0225 & 0.79 & 0.79 & 0.79 \\
\hline & & LDA & 0.84 & {$[0.8-0.88]$} & 0.0216 & 0.78 & 0.81 & 0.78 \\
\hline & & LR & 0.85 & [0.8-0.89] & 0.0224 & 0.81 & 0.81 & 0.81 \\
\hline & & NB & 0.83 & [0.78-0.87] & 0.0237 & 0.85 & 0.71 & 0.87 \\
\hline & & RF & 0.86 & [0.82-0.9] & 0.0179 & 0.75 & 0.86 & 0.74 \\
\hline & \multirow{7}{*}{ Relief } & $\mathrm{AB}$ & 0.84 & [0.8-0.88] & 0.0204 & 0.68 & 0.88 & 0.66 \\
\hline & & $\mathrm{AE}$ & 0.85 & [0.81-0.89] & 0.0211 & 0.78 & 0.81 & 0.78 \\
\hline & & LASSO & 0.83 & {$[0.79-0.88]$} & 0.0235 & 0.79 & 0.79 & 0.79 \\
\hline & & LDA & 0.85 & [0.8-0.89] & 0.0214 & 0.81 & 0.84 & 0.8 \\
\hline & & LR & 0.84 & [0.79-0.89] & 0.0231 & 0.75 & 0.84 & 0.74 \\
\hline & & NB & 0.8 & {$[0.75-0.86]$} & 0.0256 & 0.74 & 0.76 & 0.74 \\
\hline & & RF & 0.86 & [0.83-0.89] & 0.0177 & 0.8 & 0.82 & 0.8 \\
\hline & \multirow{7}{*}{ RFE } & $\mathrm{AB}$ & 0.84 & [0.79-0.88] & 0.0221 & 0.75 & 0.85 & 0.74 \\
\hline & & $\mathrm{AE}$ & 0.85 & [0.8-0.89] & 0.021 & 0.78 & 0.83 & 0.78 \\
\hline & & LASSO & 0.83 & [0.78-0.87] & 0.0234 & 0.74 & 0.81 & 0.73 \\
\hline & & LDA & 0.84 & {$[0.8-0.88]$} & 0.0212 & 0.79 & 0.8 & 0.78 \\
\hline & & LR & 0.84 & [0.8-0.89] & 0.0227 & 0.79 & 0.8 & 0.79 \\
\hline & & NB & 0.79 & [0.73-0.84] & 0.0266 & 0.69 & 0.79 & 0.68 \\
\hline & & $\mathrm{RF}$ & 0.85 & [0.81-0.89] & 0.0193 & 0.79 & 0.82 & 0.78 \\
\hline \multirow{15}{*}{ Center_15 } & \multirow{7}{*}{ ANOVA } & $\mathrm{AB}$ & 0.73 & {$[0.57-0.86]$} & 0.0719 & 0.69 & 0.8 & 0.69 \\
\hline & & $\mathrm{AE}$ & 0.76 & [0.63-0.88] & 0.0627 & 0.71 & 0.8 & 0.71 \\
\hline & & LASSO & 0.71 & [0.56-0.84] & 0.0697 & 0.62 & 0.8 & 0.62 \\
\hline & & LDA & 0.69 & {$[0.5-0.86]$} & 0.0912 & 0.69 & 0.7 & 0.69 \\
\hline & & LR & 0.72 & [0.57-0.85] & 0.0691 & 0.63 & 0.8 & 0.63 \\
\hline & & NB & 0.69 & {$[0.55-0.82]$} & 0.0671 & 0.77 & 0.6 & 0.78 \\
\hline & & $\mathrm{RF}$ & 0.74 & {$[0.62-0.86]$} & 0.061 & 0.67 & 0.8 & 0.67 \\
\hline & \multirow{7}{*}{$\mathrm{KW}$} & $\mathrm{AB}$ & 0.75 & [0.59-0.87] & 0.0667 & 0.7 & 0.8 & 0.7 \\
\hline & & $\mathrm{AE}$ & 0.77 & {$[0.65-0.88]$} & 0.0569 & 0.52 & 1 & 0.52 \\
\hline & & LASSO & 0.72 & {$[0.58-0.86]$} & 0.0721 & 0.53 & 0.9 & 0.53 \\
\hline & & LDA & 0.68 & {$[0.48-0.85]$} & 0.0906 & 0.67 & 0.7 & 0.67 \\
\hline & & LR & 0.73 & {$[0.58-0.86]$} & 0.0707 & 0.58 & 0.9 & 0.57 \\
\hline & & NB & 0.69 & [0.56-0.82] & 0.0669 & 0.77 & 0.6 & 0.77 \\
\hline & & RF & 0.73 & [0.6-0.85] & 0.0603 & 0.57 & 0.9 & 0.57 \\
\hline & Relief & $\mathrm{AB}$ & 0.75 & {$[0.61-0.86]$} & 0.0612 & 0.76 & 0.8 & 0.76 \\
\hline
\end{tabular}




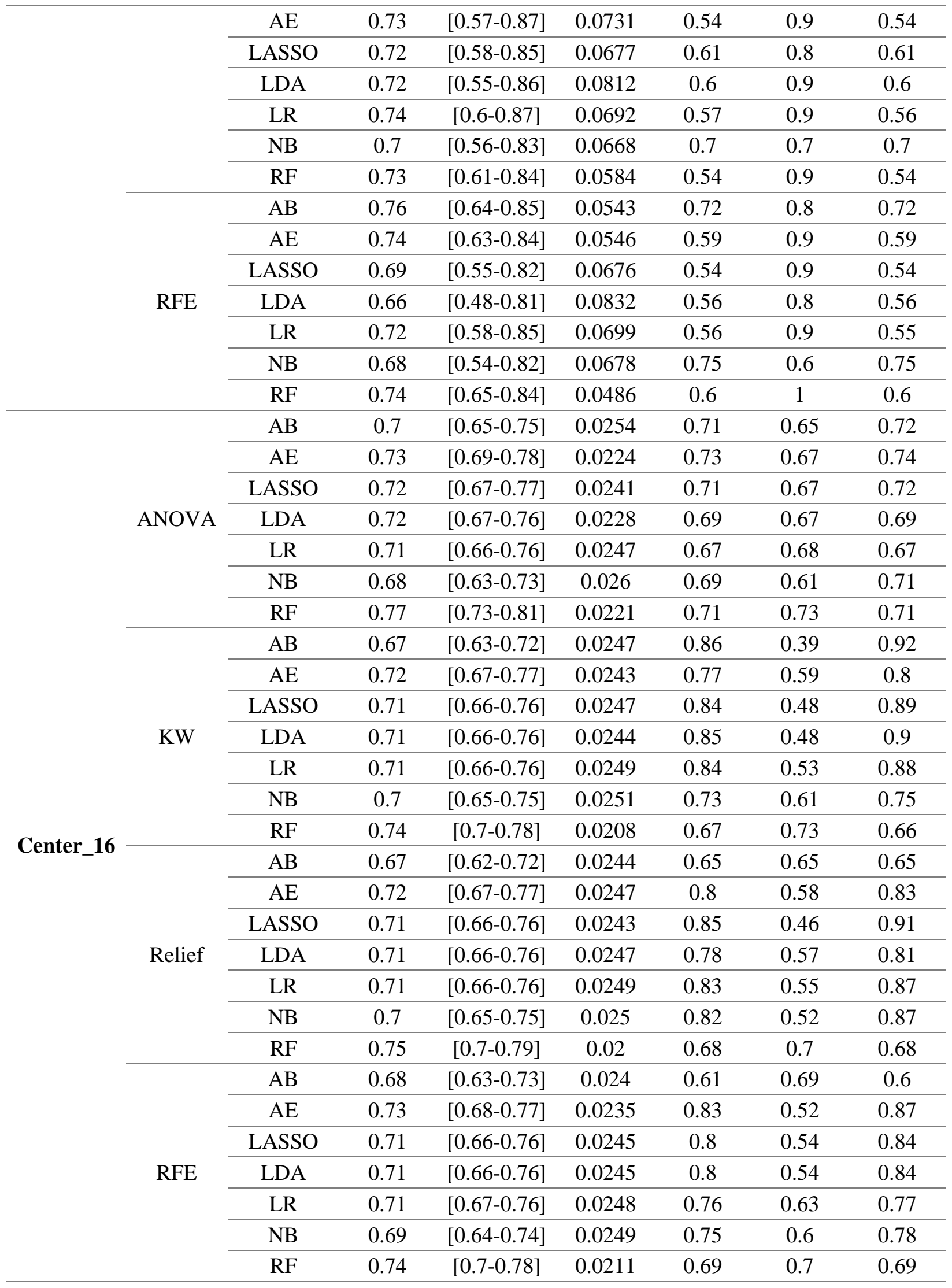




\begin{tabular}{|c|c|c|c|c|c|c|c|c|}
\hline \multirow{28}{*}{ Center_17 } & \multirow{7}{*}{ ANOVA } & $\mathrm{AB}$ & 0.69 & {$[0.65-0.74]$} & 0.025 & 0.74 & 0.6 & 0.76 \\
\hline & & $\mathrm{AE}$ & 0.7 & {$[0.65-0.75]$} & 0.0254 & 0.76 & 0.6 & 0.79 \\
\hline & & LASSO & 0.7 & {$[0.65-0.75]$} & 0.0251 & 0.74 & 0.6 & 0.77 \\
\hline & & LDA & 0.71 & [0.66-0.76] & 0.0241 & 0.8 & 0.57 & 0.83 \\
\hline & & LR & 0.7 & [0.66-0.75] & 0.0253 & 0.76 & 0.6 & 0.78 \\
\hline & & NB & 0.7 & {$[0.65-0.75]$} & 0.0252 & 0.76 & 0.6 & 0.78 \\
\hline & & $\mathrm{RF}$ & 0.77 & [0.72-0.81] & 0.0223 & 0.79 & 0.65 & 0.81 \\
\hline & \multirow{7}{*}{ KW } & $\mathrm{AB}$ & 0.67 & [0.63-0.72] & 0.0247 & 0.86 & 0.39 & 0.92 \\
\hline & & $\mathrm{AE}$ & 0.72 & [0.67-0.77] & 0.0243 & 0.77 & 0.59 & 0.8 \\
\hline & & LASSO & 0.71 & [0.66-0.76] & 0.0247 & 0.84 & 0.48 & 0.89 \\
\hline & & LDA & 0.71 & [0.66-0.76] & 0.0244 & 0.85 & 0.48 & 0.9 \\
\hline & & LR & 0.71 & [0.66-0.76] & 0.0249 & 0.84 & 0.53 & 0.88 \\
\hline & & NB & 0.7 & {$[0.65-0.75]$} & 0.0251 & 0.73 & 0.61 & 0.75 \\
\hline & & $\mathrm{RF}$ & 0.74 & [0.7-0.78] & 0.0208 & 0.67 & 0.73 & 0.66 \\
\hline & \multirow{7}{*}{ Relief } & $\mathrm{AB}$ & 0.67 & {$[0.62-0.72]$} & 0.0244 & 0.65 & 0.65 & 0.65 \\
\hline & & $\mathrm{AE}$ & 0.72 & [0.67-0.77] & 0.0247 & 0.8 & 0.58 & 0.83 \\
\hline & & LASSO & 0.71 & [0.66-0.76] & 0.0243 & 0.85 & 0.46 & 0.91 \\
\hline & & LDA & 0.71 & [0.66-0.76] & 0.0247 & 0.78 & 0.57 & 0.81 \\
\hline & & LR & 0.71 & [0.66-0.76] & 0.0249 & 0.83 & 0.55 & 0.87 \\
\hline & & NB & 0.7 & {$[0.65-0.75]$} & 0.025 & 0.82 & 0.52 & 0.87 \\
\hline & & $\mathrm{RF}$ & 0.75 & {$[0.7-0.79]$} & 0.02 & 0.68 & 0.7 & 0.68 \\
\hline & \multirow{7}{*}{ RFE } & $\mathrm{AB}$ & 0.68 & [0.63-0.73] & 0.024 & 0.61 & 0.69 & 0.6 \\
\hline & & $\mathrm{AE}$ & 0.73 & [0.68-0.77] & 0.0235 & 0.83 & 0.52 & 0.87 \\
\hline & & LASSO & 0.71 & [0.66-0.76] & 0.0245 & 0.8 & 0.54 & 0.84 \\
\hline & & LDA & 0.71 & [0.66-0.76] & 0.0245 & 0.8 & 0.54 & 0.84 \\
\hline & & LR & 0.71 & [0.67-0.76] & 0.0248 & 0.76 & 0.63 & 0.77 \\
\hline & & NB & 0.69 & [0.64-0.74] & 0.0249 & 0.75 & 0.6 & 0.78 \\
\hline & & $\mathrm{RF}$ & 0.74 & {$[0.7-0.78]$} & 0.0211 & 0.69 & 0.7 & 0.69 \\
\hline \multirow{13}{*}{ Center_18 } & \multirow{7}{*}{ ANOVA } & $\mathrm{AB}$ & 0.92 & [0.87-0.96] & 0.0232 & 0.83 & 0.91 & 0.83 \\
\hline & & $\mathrm{AE}$ & 0.91 & {$[0.85-0.96]$} & 0.0274 & 0.85 & 0.91 & 0.85 \\
\hline & & LASSO & 0.92 & [0.85-0.97] & 0.0298 & 0.86 & 0.85 & 0.86 \\
\hline & & LDA & 0.88 & [0.81-0.94] & 0.0338 & 0.79 & 0.85 & 0.79 \\
\hline & & LR & 0.92 & [0.85-0.97] & 0.0295 & 0.83 & 0.91 & 0.83 \\
\hline & & NB & 0.9 & [0.84-0.96] & 0.0287 & 0.89 & 0.82 & 0.89 \\
\hline & & $\mathrm{RF}$ & 0.69 & {$[0.57-0.8]$} & 0.0562 & 0.63 & 0.71 & 0.63 \\
\hline & \multirow{6}{*}{ KW } & $\mathrm{AB}$ & 0.92 & [0.87-0.96] & 0.0235 & 0.83 & 0.88 & 0.82 \\
\hline & & $\mathrm{AE}$ & 0.92 & [0.86-0.96] & 0.0257 & 0.88 & 0.88 & 0.88 \\
\hline & & LASSO & 0.91 & [0.85-0.96] & 0.0293 & 0.84 & 0.85 & 0.84 \\
\hline & & LDA & 0.89 & [0.83-0.94] & 0.0303 & 0.77 & 0.91 & 0.77 \\
\hline & & LR & 0.92 & [0.86-0.97] & 0.0292 & 0.83 & 0.91 & 0.83 \\
\hline & & NB & 0.9 & [0.83-0.96] & 0.0342 & 0.91 & 0.79 & 0.92 \\
\hline
\end{tabular}




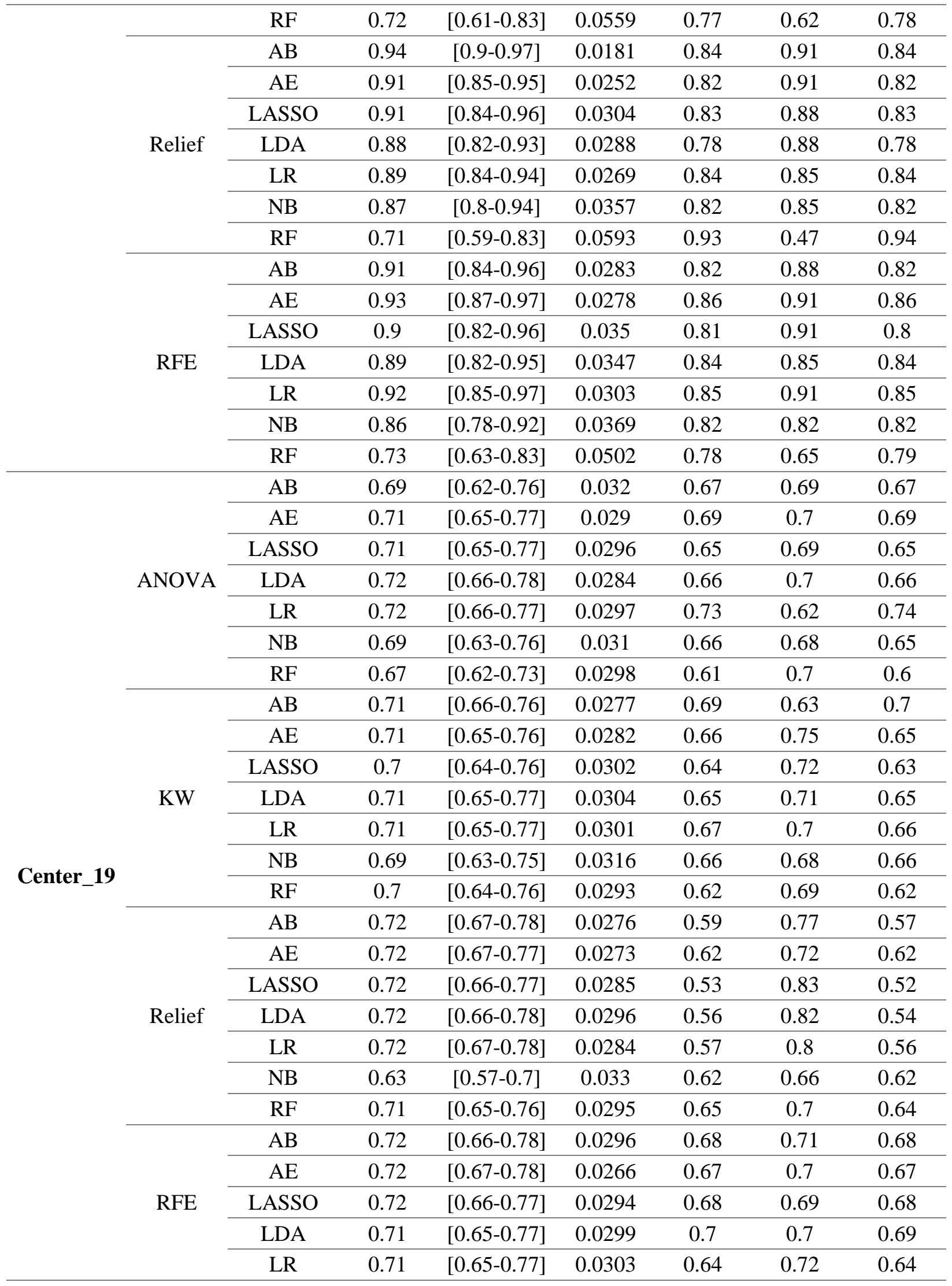




\begin{tabular}{cccccccc}
\hline NB & 0.69 & {$[0.63-0.76]$} & 0.031 & 0.66 & 0.68 & 0.66 \\
\cline { 2 - 7 } & RF & 0.7 & {$[0.64-0.75]$} & 0.0279 & 0.58 & 0.75 & 0.57 \\
\hline
\end{tabular}


Supplemental Table 14. Classification performance indices of different feature selector and classifiers in Strategy 10.

\begin{tabular}{|c|c|c|c|c|c|c|c|c|}
\hline Center & FS & Classifier & AUC & 95\% CIs & Std & Acc & Sen & Spe \\
\hline \multirow{28}{*}{ Center_01 } & \multirow{7}{*}{ ANOVA } & $\mathrm{AB}$ & 0.56 & {$[0.39-0.75]$} & 0.0927 & 0.58 & 0.64 & 0.55 \\
\hline & & $\mathrm{AE}$ & 0.66 & [0.46-0.83] & 0.0946 & 0.73 & 0.79 & 0.71 \\
\hline & & LASSO & 0.68 & {$[0.51-0.84]$} & 0.0892 & 0.71 & 0.71 & 0.71 \\
\hline & & LDA & 0.61 & {$[0.41-0.8]$} & 0.1 & 0.67 & 0.64 & 0.68 \\
\hline & & LR & 0.66 & {$[0.5-0.83]$} & 0.0879 & 0.71 & 0.64 & 0.74 \\
\hline & & NB & 0.68 & [0.51-0.85] & 0.0869 & 0.67 & 0.86 & 0.58 \\
\hline & & $\mathrm{RF}$ & 0.63 & {$[0.45-0.79]$} & 0.0858 & 0.62 & 0.71 & 0.58 \\
\hline & \multirow{7}{*}{ KW } & $\mathrm{AB}$ & 0.68 & [0.49-0.87] & 0.0967 & 0.64 & 0.86 & 0.55 \\
\hline & & $\mathrm{AE}$ & 0.68 & [0.49-0.86] & 0.0897 & 0.67 & 0.79 & 0.61 \\
\hline & & LASSO & 0.73 & [0.55-0.89] & 0.0882 & 0.76 & 0.79 & 0.74 \\
\hline & & LDA & 0.67 & {$[0.47-0.86]$} & 0.101 & 0.69 & 0.71 & 0.68 \\
\hline & & LR & 0.72 & [0.53-0.89] & 0.0923 & 0.78 & 0.71 & 0.81 \\
\hline & & NB & 0.71 & [0.54-0.87] & 0.0865 & 0.69 & 0.86 & 0.61 \\
\hline & & $\mathrm{RF}$ & 0.68 & [0.51-0.84] & 0.0831 & 0.69 & 0.71 & 0.68 \\
\hline & \multirow{7}{*}{ Relief } & $\mathrm{AB}$ & 0.61 & {$[0.43-0.8]$} & 0.0923 & 0.6 & 0.71 & 0.55 \\
\hline & & $\mathrm{AE}$ & 0.68 & {$[0.5-0.84]$} & 0.0874 & 0.62 & 0.79 & 0.55 \\
\hline & & LASSO & 0.68 & {$[0.5-0.85]$} & 0.0889 & 0.64 & 0.79 & 0.58 \\
\hline & & LDA & 0.68 & {$[0.5-0.85]$} & 0.0895 & 0.67 & 0.79 & 0.61 \\
\hline & & LR & 0.7 & {$[0.53-0.86]$} & 0.087 & 0.69 & 0.79 & 0.65 \\
\hline & & NB & 0.73 & {$[0.57-0.88]$} & 0.081 & 0.71 & 0.93 & 0.61 \\
\hline & & $\mathrm{RF}$ & 0.63 & {$[0.44-0.81]$} & 0.0942 & 0.62 & 0.71 & 0.58 \\
\hline & \multirow{7}{*}{ RFE } & $\mathrm{AB}$ & 0.63 & {$[0.45-0.8]$} & 0.0898 & 0.67 & 0.79 & 0.61 \\
\hline & & $\mathrm{AE}$ & 0.69 & {$[0.5-0.86]$} & 0.0932 & 0.71 & 0.71 & 0.71 \\
\hline & & LASSO & 0.65 & {$[0.47-0.83]$} & 0.0903 & 0.67 & 0.71 & 0.65 \\
\hline & & LDA & 0.68 & [0.49-0.84] & 0.0884 & 0.67 & 0.79 & 0.61 \\
\hline & & LR & 0.66 & {$[0.47-0.84]$} & 0.0932 & 0.67 & 0.71 & 0.65 \\
\hline & & NB & 0.68 & {$[0.51-0.85]$} & 0.0867 & 0.64 & 0.86 & 0.55 \\
\hline & & $\mathrm{RF}$ & 0.63 & {$[0.46-0.8]$} & 0.0888 & 0.6 & 0.71 & 0.55 \\
\hline \multirow{10}{*}{ Center_02 } & \multirow{7}{*}{ ANOVA } & $\mathrm{AB}$ & 0.76 & {$[0.65-0.87]$} & 0.0556 & 0.75 & 0.81 & 0.69 \\
\hline & & $\mathrm{AE}$ & 0.81 & {$[0.71-0.9]$} & 0.0482 & 0.77 & 0.76 & 0.79 \\
\hline & & LASSO & 0.81 & {$[0.71-0.9]$} & 0.0497 & 0.78 & 0.81 & 0.76 \\
\hline & & LDA & 0.8 & {$[0.7-0.9]$} & 0.0524 & 0.78 & 0.81 & 0.76 \\
\hline & & LR & 0.8 & {$[0.7-0.9]$} & 0.0508 & 0.77 & 0.78 & 0.76 \\
\hline & & NB & 0.78 & {$[0.66-0.88]$} & 0.0529 & 0.77 & 0.78 & 0.76 \\
\hline & & $\mathrm{RF}$ & 0.75 & {$[0.63-0.86]$} & 0.0572 & 0.76 & 0.76 & 0.76 \\
\hline & \multirow{3}{*}{ KW } & $\mathrm{AB}$ & 0.79 & {$[0.68-0.89]$} & 0.0522 & 0.75 & 0.86 & 0.64 \\
\hline & & $\mathrm{AE}$ & 0.81 & {$[0.7-0.9]$} & 0.0485 & 0.77 & 0.78 & 0.76 \\
\hline & & LASSO & 0.81 & {$[0.72-0.91]$} & 0.0509 & 0.78 & 0.81 & 0.76 \\
\hline
\end{tabular}




\begin{tabular}{|c|c|c|c|c|c|c|c|c|}
\hline & & LDA & 0.8 & {$[0.7-0.9]$} & 0.0523 & 0.78 & 0.81 & 0.76 \\
\hline & & LR & 0.8 & {$[0.7-0.9]$} & 0.0509 & 0.77 & 0.78 & 0.76 \\
\hline & & NB & 0.78 & [0.67-0.88] & 0.0527 & 0.77 & 0.78 & 0.76 \\
\hline & & RF & 0.75 & [0.64-0.86] & 0.0563 & 0.75 & 0.76 & 0.74 \\
\hline & & $\mathrm{AB}$ & 0.75 & [0.63-0.86] & 0.0566 & 0.73 & 0.81 & 0.67 \\
\hline & & $\mathrm{AE}$ & 0.8 & [0.7-0.9] & 0.052 & 0.77 & 0.84 & 0.71 \\
\hline & & LASSO & 0.82 & {$[0.72-0.91]$} & 0.0497 & 0.77 & 0.89 & 0.67 \\
\hline & Relief & LDA & 0.78 & [0.67-0.88] & 0.0542 & 0.76 & 0.84 & 0.69 \\
\hline & & LR & 0.81 & [0.71-0.9] & 0.0494 & 0.77 & 0.78 & 0.76 \\
\hline & & NB & 0.77 & [0.66-0.87] & 0.0538 & 0.75 & 0.76 & 0.74 \\
\hline & & RF & 0.76 & {$[0.65-0.87]$} & 0.0552 & 0.76 & 0.78 & 0.74 \\
\hline & & $\mathrm{AB}$ & 0.77 & [0.66-0.88] & 0.0538 & 0.73 & 0.78 & 0.69 \\
\hline & & $\mathrm{AE}$ & 0.81 & [0.71-0.9] & 0.0484 & 0.77 & 0.78 & 0.76 \\
\hline & & LASSO & 0.82 & {$[0.72-0.91]$} & 0.0496 & 0.8 & 0.81 & 0.79 \\
\hline & RFE & LDA & 0.82 & {$[0.72-0.91]$} & 0.0501 & 0.81 & 0.86 & 0.76 \\
\hline & & LR & 0.82 & [0.71-0.91] & 0.0497 & 0.78 & 0.81 & 0.76 \\
\hline & & NB & 0.8 & [0.69-0.89] & 0.0511 & 0.77 & 0.78 & 0.76 \\
\hline & & RF & 0.76 & [0.65-0.87] & 0.0541 & 0.72 & 0.78 & 0.67 \\
\hline & & $\mathrm{AB}$ & 0.99 & [0.98-1] & 0.0075 & 0.99 & 1 & 0.99 \\
\hline & & $\mathrm{AE}$ & 0.85 & {$[0.65-1]$} & 0.113 & 0.72 & 1 & 0.71 \\
\hline & & LASSO & 0.97 & [0.9-1] & 0.0285 & 0.94 & 1 & 0.94 \\
\hline & ANOVA & LDA & 0.97 & [0.91-1] & 0.0239 & 0.95 & 1 & 0.95 \\
\hline & & LR & 0.97 & [0.91-1] & 0.0248 & 0.95 & 1 & 0.95 \\
\hline & & NB & 0.97 & [0.92-1] & 0.0243 & 0.95 & 1 & 0.95 \\
\hline & & RF & 0.99 & [0.97-1] & 0.0082 & 0.99 & 1 & 0.99 \\
\hline & & $\mathrm{AB}$ & 0.98 & [0.95-1] & 0.0162 & 0.97 & 1 & 0.97 \\
\hline & & $\mathrm{AE}$ & 0.71 & {$[0.35-1]$} & 0.221 & 0.99 & 0.5 & 1 \\
\hline & & LASSO & 0.96 & [0.89-1] & 0.0324 & 0.93 & 1 & 0.92 \\
\hline & KW & LDA & 0.99 & [0.98-1] & 0.0076 & 0.99 & 1 & 0.99 \\
\hline Center_03 & & LR & 0.97 & [0.9-1] & 0.0284 & 0.94 & 1 & 0.94 \\
\hline & & NB & 0.95 & [0.91-0.99] & 0.0214 & 0.95 & 1 & 0.95 \\
\hline & & RF & 0.98 & [0.94-1] & 0.0165 & 0.97 & 1 & 0.97 \\
\hline & & $\mathrm{AB}$ & 0.99 & [0.96-1] & 0.0117 & 0.98 & 1 & 0.98 \\
\hline & & $\mathrm{AE}$ & 0.69 & [0.31-1] & 0.236 & 0.99 & 0.5 & 1 \\
\hline & & LASSO & 0.98 & [0.95-1] & 0.0147 & 0.98 & 1 & 0.98 \\
\hline & Relief & LDA & 0.98 & {$[0.95-1]$} & 0.0147 & 0.98 & 1 & 0.98 \\
\hline & & LR & 0.98 & [0.96-1] & 0.0128 & 0.98 & 1 & 0.98 \\
\hline & & NB & 0.99 & [0.96-1] & 0.0121 & 0.98 & 1 & 0.98 \\
\hline & & $\mathrm{RF}$ & 1 & {$[1-1]$} & 0 & 1 & 1 & 1 \\
\hline & RFF & $\mathrm{AB}$ & 0.99 & [0.98-1] & 0.0075 & 0.99 & 1 & 0.99 \\
\hline & NTE & $\mathrm{AE}$ & 0.8 & [0.53-1] & 0.153 & 0.61 & 1 & 0.6 \\
\hline
\end{tabular}




\begin{tabular}{|c|c|c|c|c|c|c|c|c|}
\hline & & LASSO & 0.98 & [0.93-1] & 0.0204 & 0.96 & 1 & 0.96 \\
\hline & & LDA & 0.98 & [0.95-1] & 0.0164 & 0.97 & 1 & 0.97 \\
\hline & & LR & 0.97 & [0.91-1] & 0.0242 & 0.95 & 1 & 0.95 \\
\hline & & NB & 0.98 & [0.95-1] & 0.0154 & 0.97 & 1 & 0.97 \\
\hline & & RF & 1 & {$[1-1]$} & 0 & 1 & 1 & 1 \\
\hline \multirow{28}{*}{ Center_04 } & \multirow{7}{*}{ ANOVA } & $\mathrm{AB}$ & 0.91 & [0.83-0.97] & 0.036 & 0.84 & 0.88 & 0.83 \\
\hline & & $\mathrm{AE}$ & 0.92 & [0.83-0.98] & 0.0385 & 0.9 & 0.92 & 0.9 \\
\hline & & LASSO & 0.92 & [0.84-0.97] & 0.0355 & 0.87 & 0.88 & 0.87 \\
\hline & & LDA & 0.9 & [0.81-0.96] & 0.0392 & 0.83 & 0.88 & 0.82 \\
\hline & & LR & 0.91 & [0.82-0.97] & 0.0392 & 0.87 & 0.88 & 0.87 \\
\hline & & NB & 0.92 & {$[0.85-0.97]$} & 0.0325 & 0.86 & 0.92 & 0.85 \\
\hline & & RF & 0.92 & [0.85-0.98] & 0.0349 & 0.89 & 0.83 & 0.91 \\
\hline & \multirow{7}{*}{$\mathrm{KW}$} & $\mathrm{AB}$ & 0.91 & [0.83-0.97] & 0.0363 & 0.8 & 0.92 & 0.77 \\
\hline & & $\mathrm{AE}$ & 0.92 & [0.83-0.98] & 0.0377 & 0.85 & 0.88 & 0.84 \\
\hline & & LASSO & 0.91 & [0.83-0.97] & 0.0364 & 0.87 & 0.88 & 0.87 \\
\hline & & LDA & 0.93 & [0.87-0.97] & 0.0259 & 0.84 & 0.88 & 0.83 \\
\hline & & LR & 0.91 & [0.82-0.97] & 0.0393 & 0.87 & 0.88 & 0.87 \\
\hline & & NB & 0.92 & [0.85-0.97] & 0.0325 & 0.86 & 0.92 & 0.85 \\
\hline & & $\mathrm{RF}$ & 0.93 & [0.86-0.98] & 0.0319 & 0.86 & 0.88 & 0.86 \\
\hline & \multirow{7}{*}{ Relief } & $\mathrm{AB}$ & 0.91 & [0.84-0.97] & 0.0325 & 0.79 & 0.96 & 0.75 \\
\hline & & $\mathrm{AE}$ & 0.91 & [0.83-0.97] & 0.0347 & 0.83 & 0.88 & 0.82 \\
\hline & & LASSO & 0.91 & [0.82-0.96] & 0.0353 & 0.85 & 0.88 & 0.84 \\
\hline & & LDA & 0.92 & [0.85-0.98] & 0.0329 & 0.87 & 0.88 & 0.87 \\
\hline & & LR & 0.91 & [0.82-0.97] & 0.0386 & 0.87 & 0.88 & 0.87 \\
\hline & & NB & 0.92 & [0.85-0.98] & 0.0326 & 0.85 & 0.92 & 0.83 \\
\hline & & $\mathrm{RF}$ & 0.92 & [0.84-0.97] & 0.0341 & 0.82 & 0.88 & 0.8 \\
\hline & \multirow{7}{*}{ RFE } & $\mathrm{AB}$ & 0.93 & [0.86-0.97] & 0.0291 & 0.87 & 0.88 & 0.87 \\
\hline & & $\mathrm{AE}$ & 0.92 & [0.83-0.98] & 0.0375 & 0.81 & 0.92 & 0.78 \\
\hline & & LASSO & 0.92 & [0.83-0.98] & 0.0361 & 0.84 & 0.92 & 0.82 \\
\hline & & LDA & 0.93 & [0.86-0.97] & 0.0277 & 0.85 & 0.92 & 0.83 \\
\hline & & LR & 0.92 & [0.85-0.97] & 0.0336 & 0.87 & 0.88 & 0.87 \\
\hline & & NB & 0.92 & [0.85-0.97] & 0.0325 & 0.86 & 0.92 & 0.85 \\
\hline & & $\mathrm{RF}$ & 0.93 & [0.87-0.98] & 0.0277 & 0.83 & 0.92 & 0.8 \\
\hline \multirow{8}{*}{ Center_05 } & \multirow{7}{*}{ ANOVA } & $\mathrm{AB}$ & 0.86 & [0.74-0.95] & 0.0524 & 0.88 & 0.71 & 0.91 \\
\hline & & $\mathrm{AE}$ & 0.86 & {$[0.78-0.93]$} & 0.0385 & 0.83 & 0.9 & 0.82 \\
\hline & & LASSO & 0.82 & {$[0.71-0.9]$} & 0.0503 & 0.82 & 0.86 & 0.82 \\
\hline & & LDA & 0.84 & [0.72-0.93] & 0.0528 & 0.83 & 0.86 & 0.83 \\
\hline & & LR & 0.83 & [0.72-0.91] & 0.0502 & 0.85 & 0.86 & 0.85 \\
\hline & & NB & 0.83 & [0.73-0.91] & 0.0453 & 0.78 & 0.86 & 0.76 \\
\hline & & $\mathrm{RF}$ & 0.9 & [0.82-0.95] & 0.0343 & 0.82 & 0.9 & 0.81 \\
\hline & KW & $\mathrm{AB}$ & 0.85 & [0.74-0.94] & 0.0502 & 0.8 & 0.86 & 0.78 \\
\hline
\end{tabular}




\begin{tabular}{|c|c|c|c|c|c|c|c|c|}
\hline & & $\mathrm{AE}$ & 0.89 & [0.81-0.95] & 0.0364 & 0.79 & 0.95 & 0.75 \\
\hline & & LASSO & 0.84 & {$[0.74-0.92]$} & 0.0453 & 0.82 & 0.9 & 0.8 \\
\hline & & LDA & 0.84 & [0.74-0.92] & 0.0468 & 0.78 & 0.95 & 0.74 \\
\hline & & LR & 0.83 & [0.74-0.91] & 0.0462 & 0.82 & 0.86 & 0.82 \\
\hline & & NB & 0.85 & [0.76-0.92] & 0.0409 & 0.78 & 0.9 & 0.75 \\
\hline & & $\mathrm{RF}$ & 0.9 & [0.82-0.95] & 0.0347 & 0.84 & 0.9 & 0.83 \\
\hline & & $\mathrm{AB}$ & 0.9 & [0.82-0.96] & 0.0382 & 0.84 & 0.9 & 0.83 \\
\hline & & $\mathrm{AE}$ & 0.87 & [0.78-0.94] & 0.0428 & 0.75 & 0.95 & 0.7 \\
\hline & & LASSO & 0.82 & [0.71-0.9] & 0.05 & 0.72 & 0.86 & 0.69 \\
\hline & Relief & LDA & 0.8 & {$[0.68-0.9]$} & 0.0544 & 0.75 & 0.81 & 0.73 \\
\hline & & LR & 0.86 & {$[0.78-0.93]$} & 0.0384 & 0.82 & 0.86 & 0.82 \\
\hline & & NB & 0.77 & [0.64-0.88] & 0.063 & 0.7 & 0.76 & 0.69 \\
\hline & & $\mathrm{RF}$ & 0.93 & [0.88-0.97] & 0.0242 & 0.87 & 0.95 & 0.85 \\
\hline & & $\mathrm{AB}$ & 0.87 & [0.76-0.95] & 0.0479 & 0.75 & 0.95 & 0.7 \\
\hline & & $\mathrm{AE}$ & 0.88 & [0.78-0.94] & 0.0395 & 0.8 & 0.9 & 0.77 \\
\hline & & LASSO & 0.8 & {$[0.69-0.9]$} & 0.0563 & 0.68 & 0.9 & 0.62 \\
\hline & RFE & LDA & 0.82 & [0.71-0.91] & 0.052 & 0.75 & 0.86 & 0.73 \\
\hline & & LR & 0.8 & [0.68-0.91] & 0.0577 & 0.75 & 0.81 & 0.73 \\
\hline & & NB & 0.83 & [0.73-0.91] & 0.0457 & 0.81 & 0.86 & 0.8 \\
\hline & & RF & 0.9 & [0.82-0.96] & 0.0329 & 0.83 & 0.86 & 0.83 \\
\hline & & $\mathrm{AB}$ & 0.84 & {$[0.75-0.91]$} & 0.0388 & 0.79 & 0.85 & 0.75 \\
\hline & & $\mathrm{AE}$ & 0.86 & [0.79-0.92] & 0.0347 & 0.79 & 0.83 & 0.76 \\
\hline & & LASSO & 0.86 & {$[0.78-0.93]$} & 0.036 & 0.82 & 0.83 & 0.82 \\
\hline & ANOVA & LDA & 0.85 & {$[0.77-0.91]$} & 0.0367 & 0.8 & 0.87 & 0.75 \\
\hline & & LR & 0.87 & [0.8-0.93] & 0.0335 & 0.81 & 0.89 & 0.76 \\
\hline & & NB & 0.85 & {$[0.77-0.91]$} & 0.0378 & 0.8 & 0.87 & 0.75 \\
\hline & & $\mathrm{RF}$ & 0.86 & [0.79-0.92] & 0.0338 & 0.81 & 0.8 & 0.81 \\
\hline & & $\mathrm{AB}$ & 0.83 & {$[0.75-0.9]$} & 0.0379 & 0.77 & 0.83 & 0.74 \\
\hline & & $\mathrm{AE}$ & 0.85 & {$[0.77-0.91]$} & 0.0367 & 0.79 & 0.87 & 0.74 \\
\hline & & LASSO & 0.87 & [0.79-0.93] & 0.0339 & 0.81 & 0.87 & 0.76 \\
\hline Center_06 & KW & LDA & 0.86 & [0.79-0.92] & 0.0347 & 0.77 & 0.93 & 0.67 \\
\hline & & LR & 0.86 & [0.78-0.92] & 0.0358 & 0.81 & 0.85 & 0.79 \\
\hline & & NB & 0.84 & {$[0.76-0.91]$} & 0.0384 & 0.75 & 0.87 & 0.68 \\
\hline & & $\mathrm{RF}$ & 0.86 & [0.78-0.92] & 0.035 & 0.78 & 0.8 & 0.76 \\
\hline & & $\mathrm{AB}$ & 0.84 & [0.76-0.9] & 0.0361 & 0.79 & 0.8 & 0.78 \\
\hline & & $\mathrm{AE}$ & 0.85 & {$[0.77-0.92]$} & 0.0356 & 0.76 & 0.91 & 0.67 \\
\hline & & LASSO & 0.88 & [0.81-0.94] & 0.0322 & 0.81 & 0.87 & 0.76 \\
\hline & Relief & LDA & 0.87 & [0.8-0.93] & 0.0328 & 0.8 & 0.85 & 0.76 \\
\hline & & LR & 0.87 & {$[0.79-0.93]$} & 0.0342 & 0.8 & 0.87 & 0.75 \\
\hline & & NB & 0.84 & [0.76-0.91] & 0.0389 & 0.79 & 0.85 & 0.75 \\
\hline & & $\mathrm{RF}$ & 0.87 & [0.79-0.93] & 0.0338 & 0.83 & 0.85 & 0.82 \\
\hline
\end{tabular}




\begin{tabular}{|c|c|c|c|c|c|c|c|c|}
\hline & \multirow{7}{*}{ RFE } & $\mathrm{AB}$ & 0.84 & {$[0.76-0.91]$} & 0.0383 & 0.77 & 0.85 & 0.72 \\
\hline & & $\mathrm{AE}$ & 0.86 & {$[0.78-0.92]$} & 0.0352 & 0.79 & 0.91 & 0.71 \\
\hline & & LASSO & 0.87 & [0.79-0.93] & 0.0341 & 0.81 & 0.87 & 0.78 \\
\hline & & LDA & 0.86 & [0.78-0.92] & 0.0359 & 0.81 & 0.87 & 0.76 \\
\hline & & LR & 0.87 & [0.79-0.93] & 0.0351 & 0.79 & 0.93 & 0.69 \\
\hline & & NB & 0.84 & [0.76-0.91] & 0.0402 & 0.79 & 0.83 & 0.76 \\
\hline & & $\mathrm{RF}$ & 0.87 & [0.8-0.93] & 0.0332 & 0.81 & 0.87 & 0.78 \\
\hline \multirow{28}{*}{ Center_07 } & \multirow{7}{*}{ ANOVA } & $\mathrm{AB}$ & 0.89 & [0.8-0.96] & 0.0442 & 0.82 & 0.91 & 0.82 \\
\hline & & $\mathrm{AE}$ & 0.65 & {$[0.47-0.81]$} & 0.0863 & 0.57 & 0.73 & 0.56 \\
\hline & & LASSO & 0.86 & [0.71-0.98] & 0.0712 & 0.85 & 0.82 & 0.85 \\
\hline & & LDA & 0.92 & [0.83-0.98] & 0.0396 & 0.88 & 0.91 & 0.88 \\
\hline & & LR & 0.92 & [0.84-0.97] & 0.0342 & 0.82 & 0.91 & 0.82 \\
\hline & & NB & 0.92 & [0.85-0.98] & 0.0343 & 0.82 & 0.91 & 0.82 \\
\hline & & $\mathrm{RF}$ & 0.88 & {$[0.77-0.98]$} & 0.0558 & 0.86 & 0.82 & 0.86 \\
\hline & \multirow{7}{*}{ KW } & $\mathrm{AB}$ & 0.94 & [0.87-0.99] & 0.031 & 0.84 & 0.91 & 0.83 \\
\hline & & $\mathrm{AE}$ & 0.61 & {$[0.45-0.77]$} & 0.0814 & 0.33 & 1 & 0.27 \\
\hline & & LASSO & 0.87 & [0.76-0.97] & 0.0538 & 0.81 & 0.82 & 0.81 \\
\hline & & LDA & 0.89 & {$[0.79-0.96]$} & 0.0435 & 0.8 & 0.82 & 0.79 \\
\hline & & LR & 0.87 & [0.76-0.97] & 0.0561 & 0.82 & 0.82 & 0.82 \\
\hline & & NB & 0.92 & [0.85-0.98] & 0.0346 & 0.82 & 0.91 & 0.81 \\
\hline & & $\mathrm{RF}$ & 0.89 & {$[0.75-0.98]$} & 0.0609 & 0.9 & 0.82 & 0.9 \\
\hline & \multirow{7}{*}{ Relief } & $\mathrm{AB}$ & 0.86 & {$[0.75-0.95]$} & 0.0511 & 0.79 & 0.91 & 0.78 \\
\hline & & $\mathrm{AE}$ & 0.8 & [0.7-0.89] & 0.049 & 0.69 & 0.91 & 0.68 \\
\hline & & LASSO & 0.92 & [0.81-0.99] & 0.0474 & 0.85 & 0.91 & 0.85 \\
\hline & & LDA & 0.89 & {$[0.8-0.97]$} & 0.0427 & 0.73 & 0.91 & 0.72 \\
\hline & & LR & 0.92 & [0.83-0.99] & 0.0413 & 0.79 & 0.91 & 0.78 \\
\hline & & NB & 0.91 & [0.83-0.98] & 0.0383 & 0.95 & 0.73 & 0.96 \\
\hline & & $\mathrm{RF}$ & 0.91 & [0.85-0.97] & 0.0318 & 0.75 & 1 & 0.73 \\
\hline & \multirow{7}{*}{ RFE } & $\mathrm{AB}$ & 0.89 & {$[0.79-0.96]$} & 0.0429 & 0.79 & 0.91 & 0.78 \\
\hline & & $\mathrm{AE}$ & 0.71 & {$[0.55-0.86]$} & 0.0764 & 0.54 & 0.91 & 0.51 \\
\hline & & LASSO & 0.86 & [0.74-0.97] & 0.0606 & 0.8 & 0.82 & 0.8 \\
\hline & & LDA & 0.9 & [0.78-0.98] & 0.0523 & 0.82 & 0.91 & 0.81 \\
\hline & & LR & 0.88 & [0.74-0.99] & 0.0645 & 0.88 & 0.82 & 0.88 \\
\hline & & NB & 0.9 & [0.81-0.97] & 0.04 & 0.79 & 0.91 & 0.78 \\
\hline & & $\mathrm{RF}$ & 0.89 & [0.76-0.98] & 0.0577 & 0.78 & 0.91 & 0.77 \\
\hline \multirow{6}{*}{ Center_08 } & \multirow{6}{*}{ ANOVA } & $\mathrm{AB}$ & 0.74 & {$[0.62-0.85]$} & 0.0574 & 0.71 & 0.81 & 0.7 \\
\hline & & $\mathrm{AE}$ & 0.7 & {$[0.55-0.84]$} & 0.0731 & 0.68 & 0.71 & 0.68 \\
\hline & & LASSO & 0.75 & [0.6-0.88] & 0.0676 & 0.75 & 0.71 & 0.76 \\
\hline & & LDA & 0.74 & [0.61-0.86] & 0.0621 & 0.71 & 0.71 & 0.71 \\
\hline & & LR & 0.75 & [0.59-0.88] & 0.0708 & 0.78 & 0.71 & 0.79 \\
\hline & & NB & 0.69 & [0.54-0.83] & 0.0724 & 0.69 & 0.67 & 0.69 \\
\hline
\end{tabular}




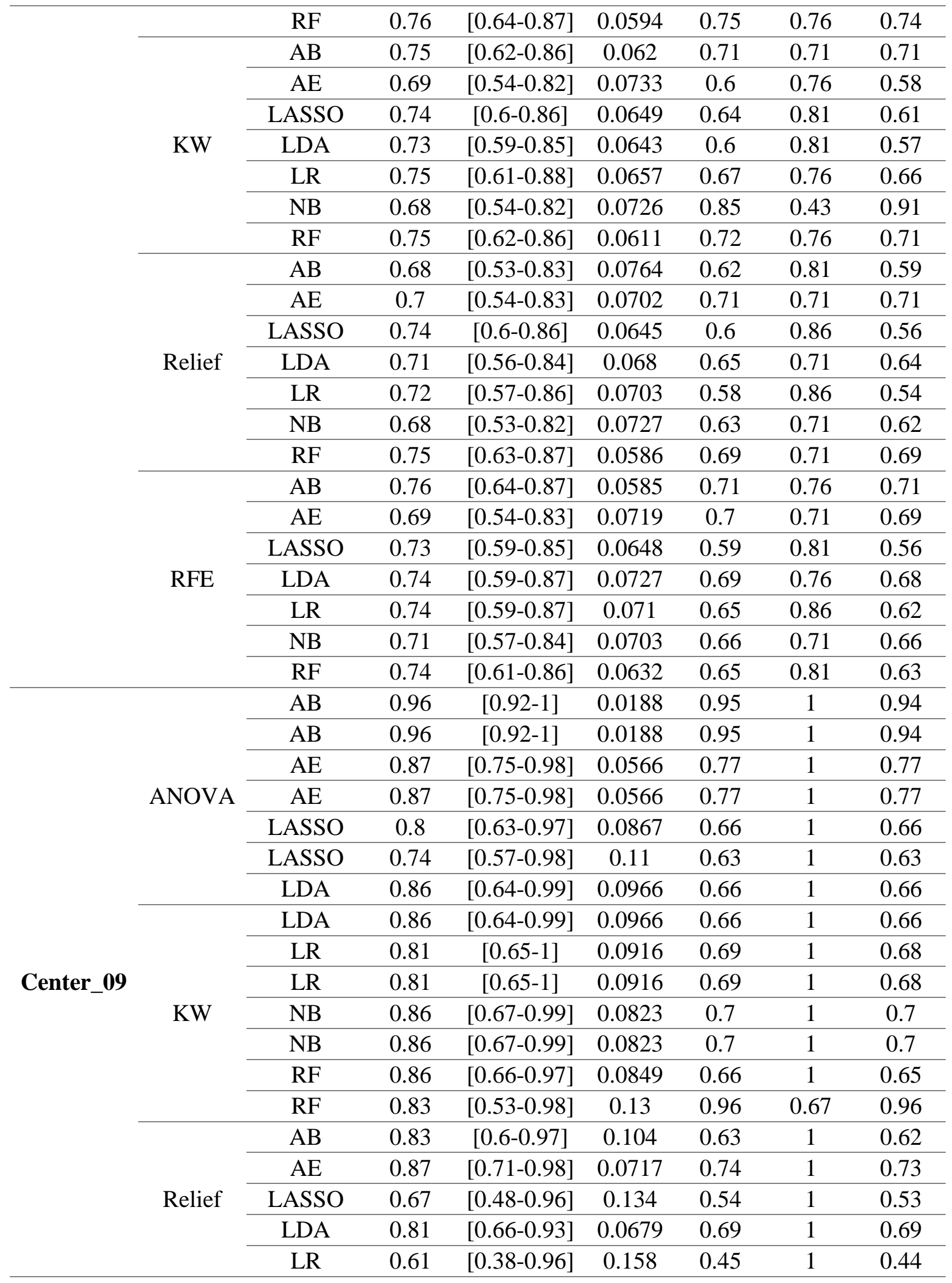




\begin{tabular}{|c|c|c|c|c|c|c|c|c|}
\hline & & NB & 0.88 & {$[0.7-0.99]$} & 0.079 & 0.73 & 1 & 0.72 \\
\hline & & $\mathrm{RF}$ & 0.84 & [0.61-0.97] & 0.0992 & 0.93 & 0.67 & 0.93 \\
\hline & \multirow{7}{*}{ RFE } & $\mathrm{AB}$ & 0.96 & [0.93-0.98] & 0.0151 & 0.96 & 1 & 0.96 \\
\hline & & $\mathrm{AE}$ & 0.87 & [0.76-0.95] & 0.0476 & 0.79 & 1 & 0.78 \\
\hline & & LASSO & 0.61 & [0.36-0.96] & 0.165 & 0.4 & 1 & 0.39 \\
\hline & & LDA & 0.88 & [0.66-0.99] & 0.0958 & 0.68 & 1 & 0.68 \\
\hline & & LR & 0.57 & [0.32-0.97] & 0.182 & 0.36 & 1 & 0.35 \\
\hline & & NB & 0.87 & [0.67-0.98] & 0.0837 & 0.7 & 1 & 0.7 \\
\hline & & $\mathrm{RF}$ & 0.83 & [0.59-0.97] & 0.103 & 0.59 & 1 & 0.58 \\
\hline \multirow{28}{*}{ Center_10 } & \multirow{7}{*}{ ANOVA } & $\mathrm{AB}$ & 0.65 & [0.52-0.77] & 0.0647 & 0.61 & 0.68 & 0.61 \\
\hline & & $\mathrm{AE}$ & 0.64 & {$[0.51-0.75]$} & 0.0628 & 0.63 & 0.72 & 0.62 \\
\hline & & LASSO & 0.65 & {$[0.52-0.78]$} & 0.0651 & 0.66 & 0.68 & 0.66 \\
\hline & & LDA & 0.65 & {$[0.52-0.77]$} & 0.0647 & 0.65 & 0.68 & 0.64 \\
\hline & & LR & 0.65 & {$[0.52-0.78]$} & 0.0659 & 0.68 & 0.68 & 0.68 \\
\hline & & NB & 0.66 & [0.53-0.77] & 0.0615 & 0.68 & 0.76 & 0.67 \\
\hline & & $\mathrm{RF}$ & 0.7 & [0.58-0.82] & 0.0587 & 0.62 & 0.76 & 0.6 \\
\hline & \multirow{7}{*}{ KW } & $\mathrm{AB}$ & 0.63 & [0.49-0.75] & 0.0664 & 0.61 & 0.68 & 0.61 \\
\hline & & $\mathrm{AE}$ & 0.69 & {$[0.56-0.82]$} & 0.0647 & 0.69 & 0.72 & 0.68 \\
\hline & & LASSO & 0.65 & {$[0.51-0.77]$} & 0.0646 & 0.61 & 0.72 & 0.59 \\
\hline & & LDA & 0.64 & {$[0.5-0.77]$} & 0.0663 & 0.62 & 0.64 & 0.62 \\
\hline & & LR & 0.63 & [0.49-0.76] & 0.0667 & 0.54 & 0.76 & 0.51 \\
\hline & & NB & 0.66 & [0.53-0.77] & 0.0621 & 0.67 & 0.72 & 0.67 \\
\hline & & $\mathrm{RF}$ & 0.7 & [0.59-0.82] & 0.0579 & 0.67 & 0.68 & 0.67 \\
\hline & \multirow{7}{*}{ Relief } & $\mathrm{AB}$ & 0.5 & [0.36-0.65] & 0.0708 & 0.55 & 0.56 & 0.55 \\
\hline & & $\mathrm{AE}$ & 0.57 & {$[0.44-0.7]$} & 0.0671 & 0.55 & 0.68 & 0.53 \\
\hline & & LASSO & 0.58 & [0.44-0.72] & 0.0725 & 0.6 & 0.64 & 0.59 \\
\hline & & LDA & 0.56 & [0.41-0.72] & 0.0773 & 0.6 & 0.64 & 0.59 \\
\hline & & LR & 0.6 & {$[0.47-0.74]$} & 0.0693 & 0.56 & 0.68 & 0.54 \\
\hline & & NB & 0.65 & [0.53-0.77] & 0.0617 & 0.55 & 0.84 & 0.51 \\
\hline & & $\mathrm{RF}$ & 0.65 & [0.54-0.77] & 0.0595 & 0.55 & 0.76 & 0.52 \\
\hline & \multirow{7}{*}{ RFE } & $\mathrm{AB}$ & 0.67 & [0.54-0.79] & 0.0636 & 0.59 & 0.72 & 0.57 \\
\hline & & $\mathrm{AE}$ & 0.65 & [0.53-0.79] & 0.0648 & 0.65 & 0.68 & 0.65 \\
\hline & & LASSO & 0.61 & [0.46-0.75] & 0.0725 & 0.85 & 0.36 & 0.92 \\
\hline & & LDA & 0.57 & [0.43-0.72] & 0.0736 & 0.58 & 0.64 & 0.57 \\
\hline & & LR & 0.62 & {$[0.47-0.76]$} & 0.0703 & 0.84 & 0.36 & 0.91 \\
\hline & & NB & 0.66 & [0.53-0.78] & 0.0613 & 0.66 & 0.76 & 0.65 \\
\hline & & $\mathrm{RF}$ & 0.69 & {$[0.59-0.8]$} & 0.0529 & 0.58 & 0.76 & 0.55 \\
\hline \multirow{4}{*}{ Center_11 } & \multirow{4}{*}{ ANOVA } & $\mathrm{AB}$ & 0.78 & [0.7-0.87] & 0.0447 & 0.77 & 0.75 & 0.77 \\
\hline & & $\mathrm{AE}$ & 0.81 & [0.74-0.88] & 0.0376 & 0.73 & 0.82 & 0.72 \\
\hline & & LASSO & 0.85 & [0.77-0.92] & 0.0368 & 0.79 & 0.79 & 0.79 \\
\hline & & LDA & 0.85 & [0.76-0.93] & 0.0424 & 0.82 & 0.82 & 0.82 \\
\hline
\end{tabular}




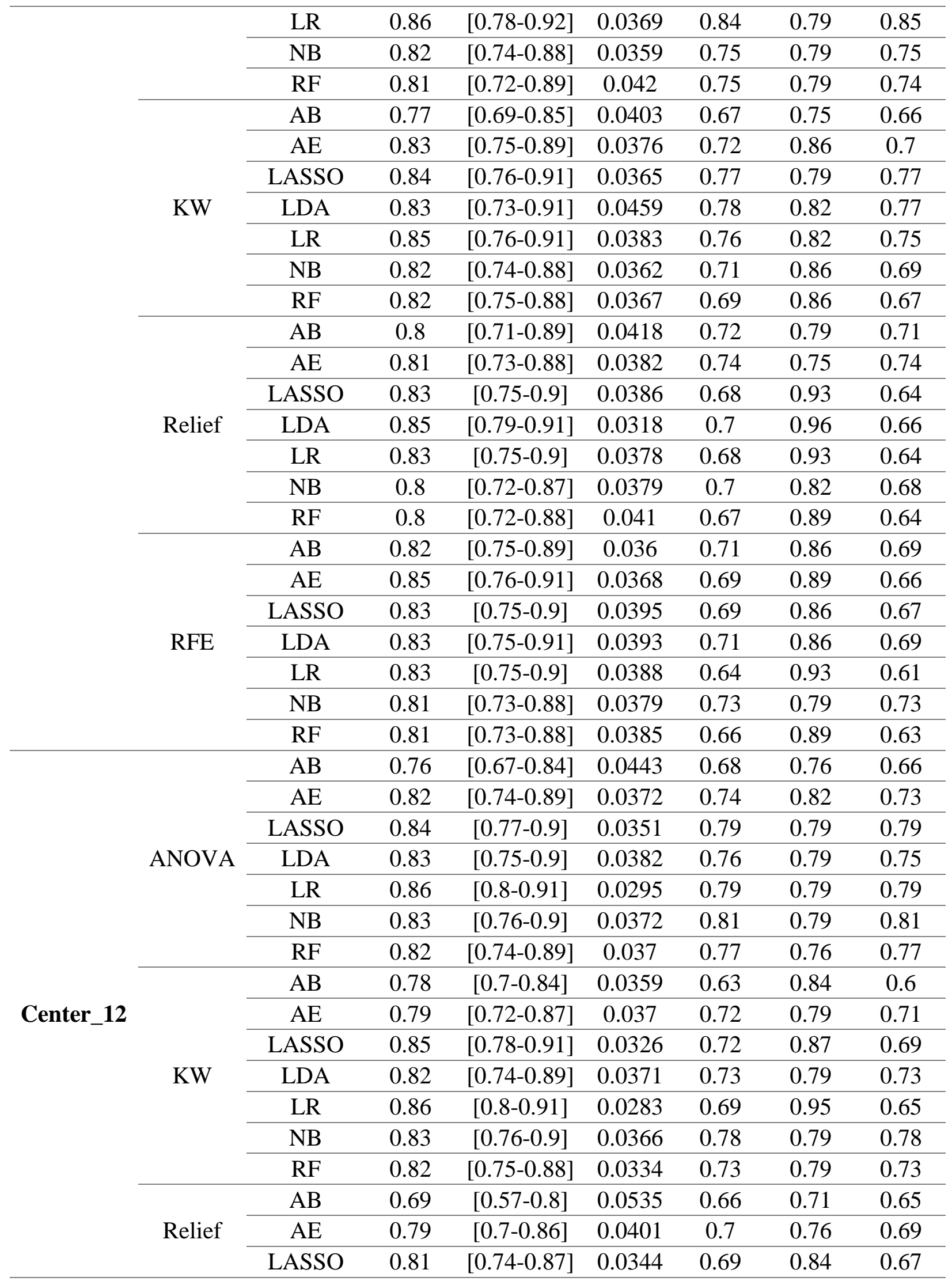




\begin{tabular}{|c|c|c|c|c|c|c|c|c|}
\hline & & LDA & 0.77 & [0.68-0.85] & 0.0411 & 0.7 & 0.74 & 0.7 \\
\hline & & LR & 0.84 & {$[0.78-0.9]$} & 0.0305 & 0.7 & 0.87 & 0.67 \\
\hline & & NB & 0.82 & {$[0.74-0.9]$} & 0.0393 & 0.74 & 0.84 & 0.72 \\
\hline & & $\mathrm{RF}$ & 0.82 & {$[0.75-0.88]$} & 0.0353 & 0.75 & 0.79 & 0.74 \\
\hline & \multirow{7}{*}{ RFE } & $\mathrm{AB}$ & 0.74 & [0.64-0.84] & 0.0512 & 0.74 & 0.74 & 0.74 \\
\hline & & $\mathrm{AE}$ & 0.79 & [0.72-0.86] & 0.038 & 0.71 & 0.82 & 0.69 \\
\hline & & LASSO & 0.82 & [0.76-0.89] & 0.0352 & 0.7 & 0.87 & 0.67 \\
\hline & & LDA & 0.74 & {$[0.65-0.82]$} & 0.0449 & 0.56 & 0.89 & 0.5 \\
\hline & & LR & 0.85 & {$[0.79-0.9]$} & 0.0297 & 0.69 & 0.95 & 0.65 \\
\hline & & NB & 0.83 & [0.76-0.91] & 0.0379 & 0.73 & 0.87 & 0.71 \\
\hline & & $\mathrm{RF}$ & 0.82 & {$[0.75-0.88]$} & 0.0345 & 0.7 & 0.87 & 0.68 \\
\hline \multirow{28}{*}{ Center_13 } & \multirow{7}{*}{ ANOVA } & $\mathrm{AB}$ & 0.82 & [0.73-0.89] & 0.0411 & 0.78 & 0.79 & 0.78 \\
\hline & & $\mathrm{AE}$ & 0.83 & [0.74-0.91] & 0.0439 & 0.82 & 0.79 & 0.82 \\
\hline & & LASSO & 0.88 & [0.82-0.94] & 0.0297 & 0.81 & 0.82 & 0.8 \\
\hline & & LDA & 0.9 & [0.84-0.94] & 0.0236 & 0.81 & 0.86 & 0.8 \\
\hline & & LR & 0.88 & [0.82-0.93] & 0.0283 & 0.79 & 0.86 & 0.78 \\
\hline & & NB & 0.83 & [0.74-0.91] & 0.0433 & 0.84 & 0.68 & 0.86 \\
\hline & & $\mathrm{RF}$ & 0.85 & {$[0.77-0.9]$} & 0.0333 & 0.77 & 0.79 & 0.77 \\
\hline & \multirow{7}{*}{ KW } & $\mathrm{AB}$ & 0.83 & [0.74-0.9] & 0.0413 & 0.67 & 0.86 & 0.65 \\
\hline & & $\mathrm{AE}$ & 0.81 & [0.72-0.89] & 0.0425 & 0.68 & 0.89 & 0.66 \\
\hline & & LASSO & 0.88 & [0.82-0.94] & 0.0297 & 0.79 & 0.86 & 0.78 \\
\hline & & LDA & 0.88 & [0.82-0.93] & 0.0292 & 0.7 & 0.93 & 0.67 \\
\hline & & LR & 0.89 & [0.83-0.94] & 0.0274 & 0.79 & 0.86 & 0.79 \\
\hline & & NB & 0.83 & [0.74-0.91] & 0.044 & 0.77 & 0.79 & 0.77 \\
\hline & & $\mathrm{RF}$ & 0.85 & [0.78-0.9] & 0.0325 & 0.68 & 0.93 & 0.66 \\
\hline & \multirow{7}{*}{ Relief } & $\mathrm{AB}$ & 0.84 & [0.72-0.92] & 0.0507 & 0.77 & 0.86 & 0.77 \\
\hline & & $\mathrm{AE}$ & 0.75 & [0.66-0.83] & 0.0445 & 0.68 & 0.75 & 0.68 \\
\hline & & LASSO & 0.88 & [0.82-0.94] & 0.0296 & 0.63 & 1 & 0.59 \\
\hline & & LDA & 0.89 & [0.85-0.93] & 0.0217 & 0.76 & 1 & 0.73 \\
\hline & & LR & 0.89 & [0.83-0.94] & 0.0266 & 0.67 & 1 & 0.64 \\
\hline & & NB & 0.79 & [0.69-0.88] & 0.048 & 0.74 & 0.75 & 0.74 \\
\hline & & $\mathrm{RF}$ & 0.84 & [0.77-0.91] & 0.0345 & 0.79 & 0.79 & 0.79 \\
\hline & \multirow{7}{*}{ RFE } & $\mathrm{AB}$ & 0.85 & [0.77-0.92] & 0.0389 & 0.82 & 0.82 & 0.82 \\
\hline & & $\mathrm{AE}$ & 0.81 & [0.73-0.89] & 0.0419 & 0.67 & 0.89 & 0.65 \\
\hline & & LASSO & 0.86 & {$[0.78-0.92]$} & 0.0366 & 0.74 & 0.89 & 0.72 \\
\hline & & LDA & 0.87 & [0.78-0.93] & 0.0405 & 0.7 & 0.93 & 0.68 \\
\hline & & LR & 0.88 & [0.81-0.94] & 0.0307 & 0.79 & 0.82 & 0.78 \\
\hline & & NB & 0.83 & [0.75-0.91] & 0.0408 & 0.87 & 0.68 & 0.89 \\
\hline & & $\mathrm{RF}$ & 0.86 & {$[0.78-0.91]$} & 0.0318 & 0.68 & 0.93 & 0.66 \\
\hline \multirow{2}{*}{ Center_14 } & \multirow{2}{*}{ ANOVA } & $\mathrm{AB}$ & 0.85 & [0.78-0.92] & 0.0347 & 0.79 & 0.79 & 0.79 \\
\hline & & $\mathrm{AE}$ & 0.87 & [0.79-0.93] & 0.0377 & 0.81 & 0.88 & 0.8 \\
\hline
\end{tabular}




\begin{tabular}{|c|c|c|c|c|c|c|c|c|}
\hline & & LASSO & 0.87 & [0.79-0.94] & 0.0365 & 0.83 & 0.88 & 0.83 \\
\hline & & LDA & 0.88 & [0.81-0.94] & 0.0351 & 0.84 & 0.88 & 0.83 \\
\hline & & LR & 0.89 & [0.82-0.95] & 0.0339 & 0.85 & 0.88 & 0.84 \\
\hline & & NB & 0.87 & [0.78-0.93] & 0.0364 & 0.83 & 0.85 & 0.83 \\
\hline & & $\mathrm{RF}$ & 0.89 & [0.84-0.94] & 0.0244 & 0.85 & 0.88 & 0.85 \\
\hline & & $\mathrm{AB}$ & 0.85 & {$[0.76-0.91]$} & 0.0381 & 0.74 & 0.94 & 0.71 \\
\hline & & $\mathrm{AE}$ & 0.86 & [0.77-0.92] & 0.0383 & 0.74 & 0.91 & 0.72 \\
\hline & & LASSO & 0.89 & [0.82-0.95] & 0.0337 & 0.85 & 0.85 & 0.85 \\
\hline & KW & LDA & 0.88 & [0.81-0.94] & 0.0324 & 0.77 & 0.94 & 0.75 \\
\hline & & LR & 0.89 & [0.82-0.95] & 0.0343 & 0.83 & 0.88 & 0.82 \\
\hline & & NB & 0.87 & {$[0.79-0.94]$} & 0.0356 & 0.81 & 0.88 & 0.8 \\
\hline & & $\mathrm{RF}$ & 0.9 & [0.85-0.93] & 0.021 & 0.76 & 0.91 & 0.74 \\
\hline & & $\mathrm{AB}$ & 0.86 & [0.8-0.92] & 0.0309 & 0.77 & 0.94 & 0.75 \\
\hline & & $\mathrm{AE}$ & 0.83 & [0.76-0.9] & 0.0372 & 0.74 & 0.85 & 0.73 \\
\hline & & LASSO & 0.89 & [0.82-0.94] & 0.034 & 0.81 & 0.88 & 0.8 \\
\hline & Relief & LDA & 0.89 & [0.83-0.94] & 0.03 & 0.83 & 0.88 & 0.82 \\
\hline & & LR & 0.89 & [0.82-0.95] & 0.0344 & 0.82 & 0.88 & 0.81 \\
\hline & & NB & 0.86 & [0.79-0.92] & 0.0349 & 0.82 & 0.85 & 0.82 \\
\hline & & $\mathrm{RF}$ & 0.9 & {$[0.85-0.94]$} & 0.021 & 0.83 & 0.88 & 0.82 \\
\hline & & $\mathrm{AB}$ & 0.86 & {$[0.8-0.92]$} & 0.0324 & 0.78 & 0.94 & 0.76 \\
\hline & & $\mathrm{AE}$ & 0.86 & {$[0.78-0.92]$} & 0.0373 & 0.82 & 0.82 & 0.82 \\
\hline & & LASSO & 0.88 & [0.81-0.94] & 0.0348 & 0.8 & 0.88 & 0.79 \\
\hline & RFE & LDA & 0.88 & [0.81-0.93] & 0.0315 & 0.78 & 0.94 & 0.76 \\
\hline & & LR & 0.88 & [0.81-0.94] & 0.0348 & 0.81 & 0.91 & 0.79 \\
\hline & & NB & 0.87 & [0.79-0.92] & 0.0349 & 0.88 & 0.79 & 0.89 \\
\hline & & $\mathrm{RF}$ & 0.9 & {$[0.85-0.94]$} & 0.0233 & 0.82 & 0.91 & 0.81 \\
\hline & & $\mathrm{AB}$ & 0.55 & [0.29-0.73] & 0.119 & 0.66 & 0.67 & 0.66 \\
\hline & & $\mathrm{AE}$ & 0.9 & {$[0.85-0.95]$} & 0.0253 & 0.87 & 1 & 0.87 \\
\hline & & LASSO & 0.65 & {$[0.58-0.74]$} & 0.0421 & 0.62 & 1 & 0.62 \\
\hline & ANOVA & LDA & 0.75 & [0.64-0.88] & 0.0623 & 0.65 & 1 & 0.65 \\
\hline & & LR & 0.68 & [0.59-0.77] & 0.0457 & 0.62 & 1 & 0.61 \\
\hline & & NB & 0.48 & [0.42-0.55] & 0.0348 & 0.45 & 1 & 0.44 \\
\hline & & $\mathrm{RF}$ & 0.69 & [0.61-0.79] & 0.0493 & 0.61 & 1 & 0.61 \\
\hline Center_15 & & $\mathrm{AB}$ & 0.43 & [0.34-0.51] & 0.0428 & 0.37 & 1 & 0.36 \\
\hline & & $\mathrm{AE}$ & 0.96 & [0.92-0.98] & 0.0154 & 0.93 & 1 & 0.93 \\
\hline & & LASSO & 0.69 & {$[0.62-0.76]$} & 0.0362 & 0.64 & 1 & 0.64 \\
\hline & KW & LDA & 0.73 & {$[0.62-0.9]$} & 0.0757 & 0.65 & 1 & 0.64 \\
\hline & & LR & 0.67 & [0.59-0.75] & 0.0427 & 0.62 & 1 & 0.61 \\
\hline & & NB & 0.53 & {$[0.39-0.73]$} & 0.0908 & 0.41 & 1 & 0.4 \\
\hline & & $\mathrm{RF}$ & 0.71 & {$[0.6-0.82]$} & 0.054 & 0.58 & 1 & 0.57 \\
\hline & Relief & $\mathrm{AB}$ & 0.53 & {$[0.35-0.75]$} & 0.106 & 0.36 & 1 & 0.35 \\
\hline
\end{tabular}




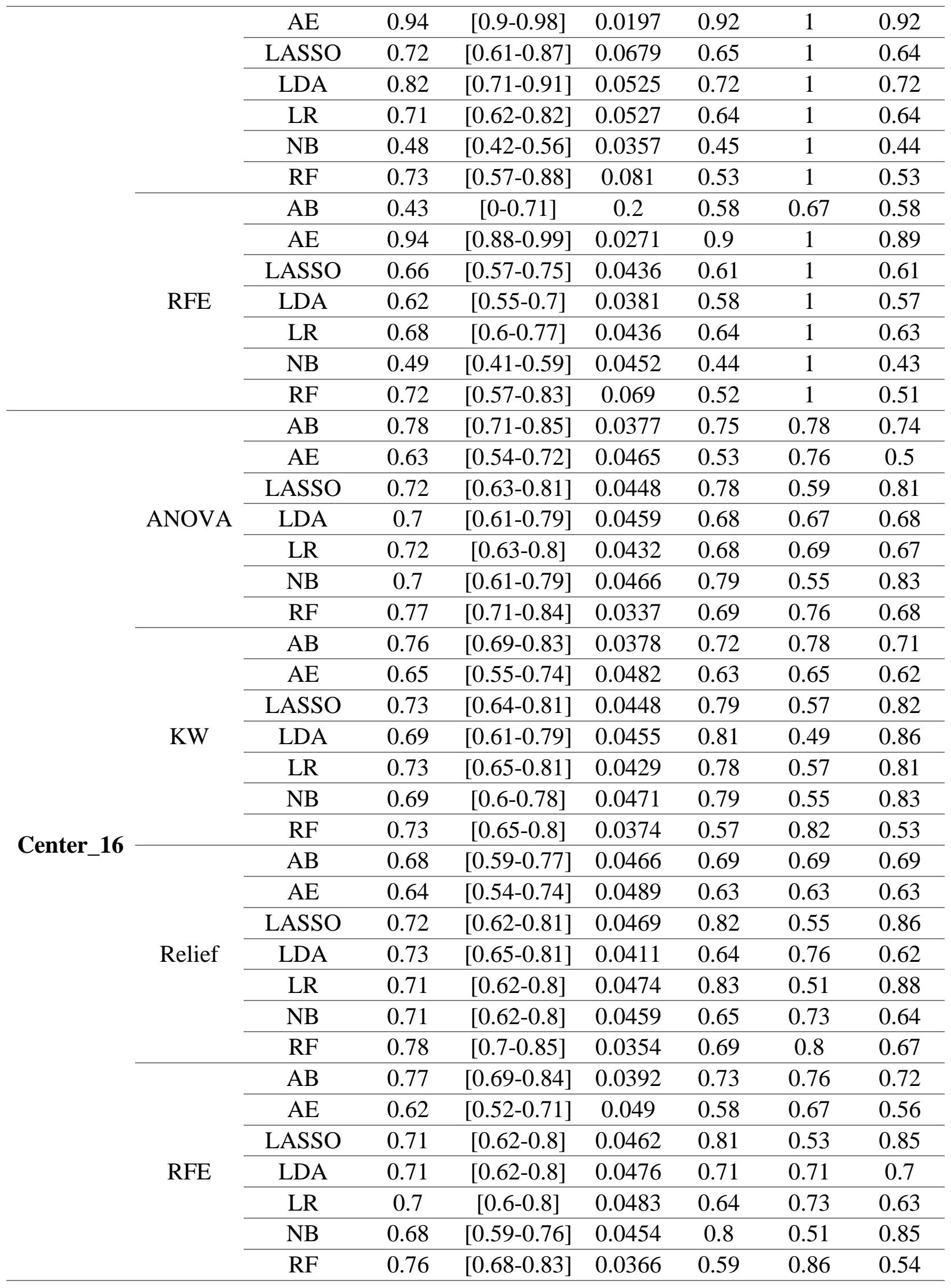




\begin{tabular}{|c|c|c|c|c|c|c|c|c|}
\hline \multirow{28}{*}{ Center_17 } & \multirow{7}{*}{ ANOVA } & $\mathrm{AB}$ & 0.74 & [0.66-0.81] & 0.0377 & 0.71 & 0.71 & 0.71 \\
\hline & & $\mathrm{AE}$ & 0.66 & {$[0.58-0.74]$} & 0.0413 & 0.62 & 0.68 & 0.61 \\
\hline & & LASSO & 0.77 & {$[0.7-0.84]$} & 0.0357 & 0.73 & 0.75 & 0.73 \\
\hline & & LDA & 0.75 & [0.66-0.83] & 0.042 & 0.73 & 0.75 & 0.72 \\
\hline & & LR & 0.75 & [0.68-0.82] & 0.0361 & 0.71 & 0.73 & 0.71 \\
\hline & & NB & 0.73 & {$[0.66-0.81]$} & 0.0387 & 0.7 & 0.73 & 0.69 \\
\hline & & $\mathrm{RF}$ & 0.78 & {$[0.71-0.84]$} & 0.0338 & 0.77 & 0.68 & 0.79 \\
\hline & \multirow{7}{*}{ KW } & $\mathrm{AB}$ & 0.69 & [0.62-0.77] & 0.0382 & 0.82 & 0.43 & 0.89 \\
\hline & & $\mathrm{AE}$ & 0.73 & [0.65-0.81] & 0.0404 & 0.68 & 0.71 & 0.67 \\
\hline & & LASSO & 0.73 & {$[0.66-0.81]$} & 0.04 & 0.69 & 0.71 & 0.68 \\
\hline & & LDA & 0.72 & [0.63-0.79] & 0.0413 & 0.69 & 0.71 & 0.68 \\
\hline & & LR & 0.71 & [0.64-0.79] & 0.0378 & 0.6 & 0.79 & 0.56 \\
\hline & & NB & 0.68 & {$[0.6-0.76]$} & 0.0417 & 0.66 & 0.71 & 0.65 \\
\hline & & $\mathrm{RF}$ & 0.77 & {$[0.7-0.84]$} & 0.0352 & 0.68 & 0.79 & 0.66 \\
\hline & \multirow{7}{*}{ Relief } & $\mathrm{AB}$ & 0.67 & {$[0.58-0.75]$} & 0.0412 & 0.56 & 0.76 & 0.52 \\
\hline & & $\mathrm{AE}$ & 0.76 & {$[0.68-0.84]$} & 0.0396 & 0.68 & 0.79 & 0.66 \\
\hline & & LASSO & 0.74 & [0.66-0.81] & 0.0371 & 0.7 & 0.76 & 0.69 \\
\hline & & LDA & 0.75 & {$[0.68-0.82]$} & 0.0372 & 0.68 & 0.75 & 0.67 \\
\hline & & LR & 0.74 & {$[0.67-0.81]$} & 0.036 & 0.67 & 0.73 & 0.66 \\
\hline & & NB & 0.68 & {$[0.6-0.77]$} & 0.0439 & 0.8 & 0.57 & 0.85 \\
\hline & & $\mathrm{RF}$ & 0.77 & {$[0.7-0.84]$} & 0.0347 & 0.68 & 0.78 & 0.66 \\
\hline & \multirow{7}{*}{ RFE } & $\mathrm{AB}$ & 0.69 & {$[0.61-0.76]$} & 0.0389 & 0.64 & 0.76 & 0.61 \\
\hline & & $\mathrm{AE}$ & 0.76 & {$[0.68-0.84]$} & 0.0378 & 0.86 & 0.56 & 0.92 \\
\hline & & LASSO & 0.73 & {$[0.65-0.8]$} & 0.0391 & 0.67 & 0.75 & 0.65 \\
\hline & & LDA & 0.75 & {$[0.67-0.82]$} & 0.0383 & 0.7 & 0.79 & 0.68 \\
\hline & & LR & 0.74 & [0.66-0.81] & 0.0373 & 0.65 & 0.78 & 0.62 \\
\hline & & NB & 0.69 & [0.61-0.77] & 0.0423 & 0.76 & 0.62 & 0.78 \\
\hline & & $\mathrm{RF}$ & 0.77 & [0.7-0.84] & 0.035 & 0.72 & 0.73 & 0.72 \\
\hline \multirow{13}{*}{ Center_18 } & \multirow{7}{*}{ ANOVA } & $\mathrm{AB}$ & 0.99 & {$[0.97-1]$} & 0.0065 & 0.95 & 1 & 0.95 \\
\hline & & $\mathrm{AE}$ & 0.96 & [0.92-0.99] & 0.0192 & 0.93 & 0.9 & 0.93 \\
\hline & & LASSO & 0.99 & [0.96-1] & 0.0097 & 0.91 & 1 & 0.91 \\
\hline & & LDA & 0.96 & [0.92-0.99] & 0.0165 & 0.95 & 0.9 & 0.95 \\
\hline & & LR & 0.98 & {$[0.95-1]$} & 0.0111 & 0.9 & 1 & 0.9 \\
\hline & & NB & 0.96 & [0.93-0.99] & 0.0148 & 0.95 & 0.9 & 0.95 \\
\hline & & $\mathrm{RF}$ & 0.95 & {$[0.88-1]$} & 0.0331 & 0.96 & 0.9 & 0.96 \\
\hline & \multirow{6}{*}{$\mathrm{KW}$} & $\mathrm{AB}$ & 1 & [0.99-1] & 0.0027 & 0.99 & 1 & 0.99 \\
\hline & & $\mathrm{AE}$ & 0.98 & [0.96-1] & 0.0106 & 0.91 & 1 & 0.91 \\
\hline & & LASSO & 0.99 & [0.97-1] & 0.0075 & 0.94 & 1 & 0.93 \\
\hline & & LDA & 0.98 & [0.96-1] & 0.008 & 0.93 & 1 & 0.93 \\
\hline & & LR & 0.99 & {$[0.96-1]$} & 0.0099 & 0.92 & 1 & 0.92 \\
\hline & & NB & 0.96 & {$[0.92-0.98]$} & 0.0151 & 0.87 & 1 & 0.86 \\
\hline
\end{tabular}




\begin{tabular}{|c|c|c|c|c|c|c|c|c|}
\hline & & $\mathrm{RF}$ & 0.98 & [0.94-1] & 0.015 & 0.86 & 1 & 0.86 \\
\hline & \multirow{7}{*}{ Relief } & $\mathrm{AB}$ & 1 & [0.99-1] & 0.0021 & 0.99 & 1 & 0.99 \\
\hline & & $\mathrm{AE}$ & 0.98 & [0.95-1] & 0.0114 & 0.91 & 1 & 0.9 \\
\hline & & LASSO & 0.98 & [0.95-1] & 0.013 & 0.89 & 1 & 0.89 \\
\hline & & LDA & 0.98 & [0.96-1] & 0.0094 & 0.91 & 1 & 0.91 \\
\hline & & LR & 0.98 & [0.95-1] & 0.0137 & 0.87 & 1 & 0.87 \\
\hline & & NB & 0.95 & [0.91-0.98] & 0.0178 & 0.86 & 1 & 0.85 \\
\hline & & $\mathrm{RF}$ & 0.98 & [0.95-1] & 0.0134 & 0.89 & 1 & 0.89 \\
\hline & \multirow{7}{*}{ RFE } & $\mathrm{AB}$ & 1 & [0.99-1] & 0.0027 & 0.99 & 1 & 0.99 \\
\hline & & $\mathrm{AE}$ & 0.98 & [0.96-1] & 0.0113 & 0.91 & 1 & 0.91 \\
\hline & & LASSO & 0.98 & [0.96-1] & 0.0102 & 0.92 & 1 & 0.92 \\
\hline & & LDA & 0.99 & [0.97-1] & 0.0079 & 0.93 & 1 & 0.92 \\
\hline & & LR & 0.99 & [0.96-1] & 0.01 & 0.91 & 1 & 0.9 \\
\hline & & NB & 0.97 & [0.94-0.99] & 0.013 & 0.88 & 1 & 0.87 \\
\hline & & $\mathrm{RF}$ & 0.98 & [0.96-1] & 0.0096 & 0.91 & 1 & 0.91 \\
\hline \multirow{26}{*}{ Center_19 } & \multirow{7}{*}{ ANOVA } & $\mathrm{AB}$ & 0.72 & {$[0.6-0.81]$} & 0.0515 & 0.69 & 0.81 & 0.68 \\
\hline & & $\mathrm{AE}$ & 0.73 & [0.62-0.82] & 0.0512 & 0.68 & 0.69 & 0.68 \\
\hline & & LASSO & 0.69 & {$[0.6-0.77]$} & 0.0424 & 0.71 & 0.69 & 0.71 \\
\hline & & LDA & 0.74 & [0.65-0.83] & 0.046 & 0.71 & 0.72 & 0.71 \\
\hline & & LR & 0.73 & [0.64-0.82] & 0.0461 & 0.63 & 0.78 & 0.62 \\
\hline & & NB & 0.71 & {$[0.61-0.8]$} & 0.0505 & 0.55 & 0.84 & 0.53 \\
\hline & & $\mathrm{RF}$ & 0.79 & {$[0.7-0.88]$} & 0.0445 & 0.8 & 0.75 & 0.8 \\
\hline & \multirow{7}{*}{ KW } & $\mathrm{AB}$ & 0.68 & {$[0.57-0.78]$} & 0.0539 & 0.59 & 0.72 & 0.58 \\
\hline & & $\mathrm{AE}$ & 0.72 & {$[0.63-0.82]$} & 0.0486 & 0.67 & 0.75 & 0.67 \\
\hline & & LASSO & 0.74 & {$[0.65-0.81]$} & 0.0398 & 0.62 & 0.81 & 0.61 \\
\hline & & LDA & 0.73 & [0.62-0.81] & 0.05 & 0.68 & 0.72 & 0.68 \\
\hline & & LR & 0.75 & [0.66-0.83] & 0.0413 & 0.62 & 0.84 & 0.6 \\
\hline & & NB & 0.72 & {$[0.62-0.8]$} & 0.0456 & 0.53 & 0.84 & 0.51 \\
\hline & & $\mathrm{RF}$ & 0.78 & [0.71-0.86] & 0.0387 & 0.71 & 0.72 & 0.71 \\
\hline & \multirow{7}{*}{ Relief } & $\mathrm{AB}$ & 0.69 & [0.59-0.78] & 0.0498 & 0.68 & 0.72 & 0.68 \\
\hline & & $\mathrm{AE}$ & 0.68 & {$[0.58-0.77]$} & 0.0488 & 0.61 & 0.72 & 0.6 \\
\hline & & LASSO & 0.74 & [0.65-0.82] & 0.0415 & 0.6 & 0.88 & 0.58 \\
\hline & & LDA & 0.76 & {$[0.67-0.85]$} & 0.0448 & 0.64 & 0.81 & 0.63 \\
\hline & & LR & 0.74 & [0.65-0.82] & 0.0427 & 0.61 & 0.81 & 0.6 \\
\hline & & NB & 0.69 & [0.6-0.79] & 0.0485 & 0.59 & 0.72 & 0.58 \\
\hline & & $\mathrm{RF}$ & 0.78 & {$[0.7-0.85]$} & 0.0388 & 0.69 & 0.84 & 0.67 \\
\hline & \multirow{5}{*}{ RFE } & $\mathrm{AB}$ & 0.71 & {$[0.61-0.8]$} & 0.0495 & 0.58 & 0.81 & 0.57 \\
\hline & & $\mathrm{AE}$ & 0.7 & [0.6-0.8] & 0.0503 & 0.68 & 0.69 & 0.68 \\
\hline & & LASSO & 0.76 & {$[0.67-0.84]$} & 0.0432 & 0.63 & 0.78 & 0.62 \\
\hline & & LDA & 0.75 & [0.64-0.84] & 0.0489 & 0.64 & 0.78 & 0.62 \\
\hline & & LR & 0.74 & [0.64-0.82] & 0.0437 & 0.54 & 0.84 & 0.52 \\
\hline
\end{tabular}




\begin{tabular}{cccccccc}
\hline NB & 0.72 & {$[0.63-0.8]$} & 0.0446 & 0.53 & 0.84 & 0.5 \\
\hline RF & 0.77 & {$[0.69-0.86]$} & 0.0418 & 0.65 & 0.78 & 0.64 \\
\hline
\end{tabular}

